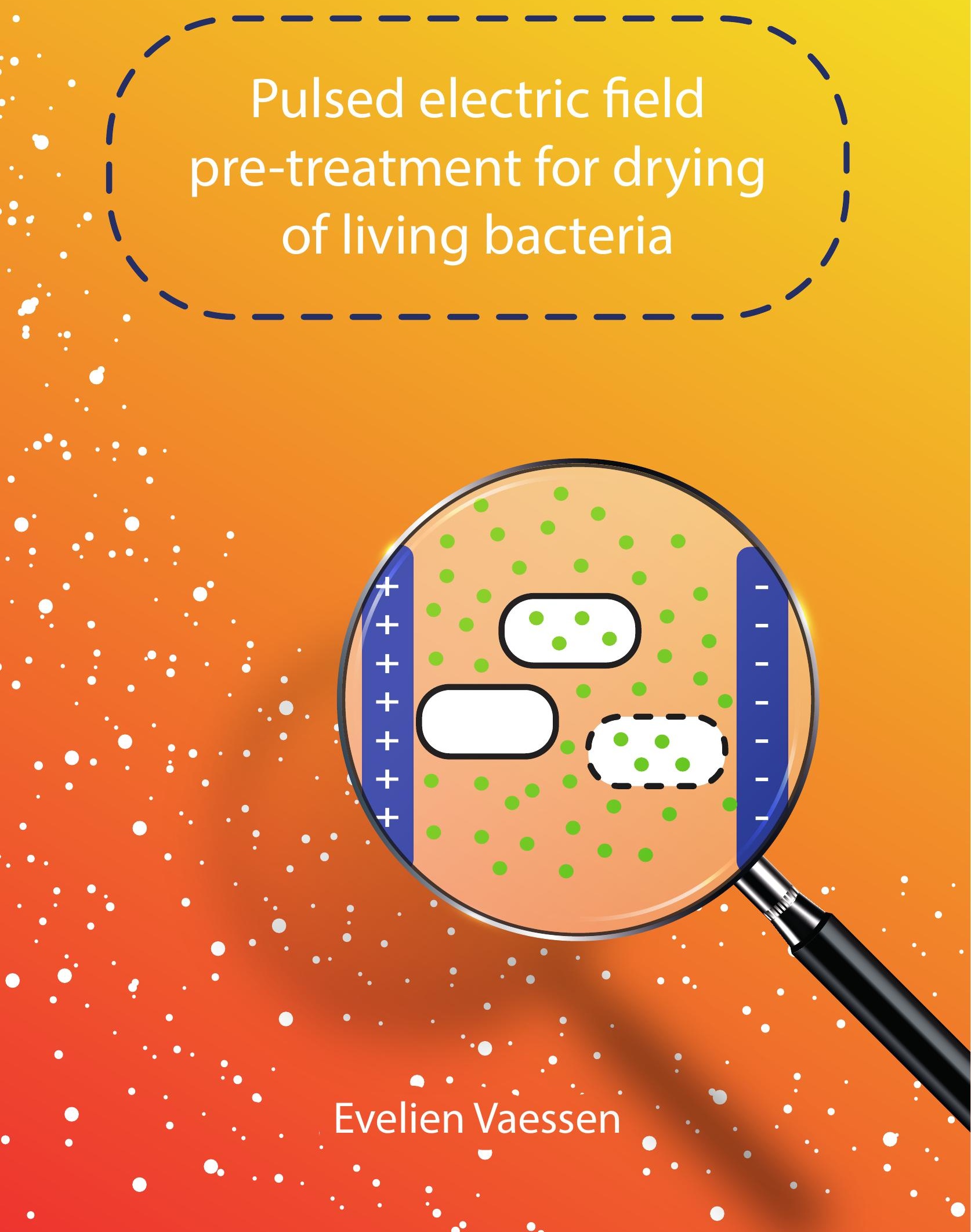





\section{Propositions}

1. Pulsed electric field treatment is an efficient method to increase intracellular sugar concentrations in reversible permeabilized cells. (this thesis)

2. The carrier matrix is key in developing a production process of dried bacteria, both with and without PEF pre-treatment. (this thesis)

3. Probiotics should be valued more by healthcare professionals instead of being a food hype.

4. An appendix to research articles with reflection on the used methods will support scientific progress.

5. From a health and environmental perspective many flexitarians is better than a few vegans.

6. Breaks together with colleagues are the most undervalued part of a working day.

Propositions belonging to the thesis entitled:

'Pulsed electric field pre-treatment for drying of living bacteria'

Evelien M.J. Vaessen

Wageningen, 12 October 2020 



\section{Pulsed electric field pre-treatment for drying of living bacteria}

Evelien M.J. Vaessen 


\section{Thesis committee}

\section{Promotor}

Dr Maarten A.I. Schutyser

Associate professor, Food Process Engineering

Wageningen University \& Research

\section{Co-promotor}

Dr Heidy M.W. den Besten

Associate professor, Laboratory of Food Microbiology

Wageningen University \& Research

\section{Other members}

Prof. Dr M. Kleerebezem, Wageningen University \& Research

Prof. Dr R. Jeantet, Agrocampus Ouest, Rennes, France

Dr M.B. Fox, FrieslandCampina, Wageningen

Dr M.N. Nierop Groot, Wageningen University \& Research

This research was conducted under the auspices of the Graduate School VLAG (Advanced studies in Food Technology, Agrobiotechnology, Nutrition and Health Sciences). 


\title{
Pulsed electric field pre-treatment for drying of living bacteria
}

\author{
Evelien M.J. Vaessen
}

\section{Thesis}

submitted in fulfilment of the requirements for the degree of doctor

at Wageningen University

by the authority of the Rector Magnificus,

Prof. Dr A.P.J. Mol,

in the presence of the

Thesis Committee appointed by the Academic Board

to be defended in public

on Monday 12 October 2020

at 1:30 p.m. in the Aula 
Evelien M.J. Vaessen

Pulsed electric field pre-treatment for drying of living bacteria 168 pages

PhD thesis, Wageningen University, Wageningen, the Netherlands (2020) With references, with summaries in English and Dutch

ISBN 978-94-6395-357-3

DOI $10.18174 / 518278$ 


\section{Contents}

$\begin{array}{lll}\text { Chapter } 1 \text { General introduction } & 7\end{array}$

Chapter 2 Pulsed electric field for increasing intracellular trehalose content in 17 Lactobacillus plantarum WCFS1

Chapter 3 Reversibility of membrane permeabilization in Lactobacillus

33 plantarum WCFS1 after pulsed electric field treatment

Chapter 4 Accumulation of intracellular trehalose and lactose in Lactobacillus 57 plantarum WCFS1 during pulsed electric field treatment and subsequent freeze and spray drying

Chapter 5 Pulsed electric field pre-treatment for enhanced bacterial 75 robustness during drying: Effect of carrier matrix and strain variability

Chapter 6 Protective effect of carrier matrices on survival of Lactobacillus 95 plantarum WCFS1 during single droplet drying explained by particle morphology development

Chapter 7 General discussion

References

Summary 149

Samenvatting 153

Acknowledgements

About the author 161

Publications

Training activities 



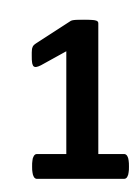

General introduction 


\subsection{Introduction}

Bacteria are commonly used in food industry as starter cultures for fermentation or as probiotic supplements. Lactic acid bacteria are among the most well-known groups of bacteria used for these purposes. Lactic acid bacteria are Gram positive, rod or cocci shaped microorganisms that produce lactic acid as the major metabolic end product of their carbohydrate fermentation. This fermentation process is used to produce common food products such as yoghurt, cheese, pickles, sausages and sauerkraut. Bacteria are not only used to produce our food; they are also important for our health. Our body contains many bacteria, of which most are present in the colon (Sender et al., 2016). In the colon these bacteria digest part of our food and produce vitamins and other nutrients that are important for our health. Benefits upon consumption of these beneficial bacteria, also referred to as probiotics, have been reported for several health issues, for example for antibiotic associated diarrhoea (Kleerebezem et al., 2019; Nagpal et al., 2012; Sanders et al., 2018). Probiotics are defined as "live microorganisms that, when administered in adequate amounts, confer a health benefit on the host" (Hill et al., 2014). As the definition already mentions, probiotics are living microorganisms. Therefore, probiotic products should contain large numbers of living bacteria, generally around $>10^{6}-10^{8}$ colony forming units (CFU) per gram or an intake of $>10^{8}-10^{10}$ CFU per day (Champagne et al., 2011; Forssten et al., 2011).

Production of living bacterial cultures requires growth of the cultures followed by subsequent storage until the bacteria are used as fermentation starter or probiotic supplement. Living bacteria can be stored frozen or in the form of a dry powder. Frozen storage requires low temperatures across the entire supply chain. Alternatively, formulation of the cultures as a dry powder can be beneficial in terms of costs and energy efficiency (Pénicaud et al., 2018). However, viability of living bacteria decreases during drying and subsequent storage due to the harsh processing and storage conditions. Minimizing this decrease in bacterial viability during drying and subsequent storage is important for energy efficient production of bacterial food ingredients.

\subsection{Drying of bacteria}

Drying is often applied to improve shelf life of food ingredients. Freeze and spray drying are the most commonly used drying processes in food industry. In general, spray drying is less time and energy consuming compared to freeze drying and therefore less costly. 
Santivarangka et al. (2007) estimated the costs of spray drying processes to be maximum one fifth of the costs of a freeze drying process. However, spray drying does expose the product to higher temperatures compared to freeze drying (Huang et al., 2017; Santivarangkna et al., 2007). During both drying processes inactivation of living bacteria can occur due to dehydration and/or thermal stresses (Lievense et al., 1992; Perdana et al., 2013).

Many factors can influence the survival of bacteria after drying, such as pre-culture conditions, drying conditions and matrix, and storage conditions afterwards. For example, bacteria in the stationary growth phase survive better during subsequent drying compared to bacteria in the exponential growth phase (Broeckx et al., 2019; Corcoran et al., 2004). Furthermore, pre-conditioning towards stresses, e.g. osmotic stress and heat shock, can increase robustness during spray drying (Desmond et al., 2002; Gaucher et al., 2019).

Many studies focus on strategies to increase survival of bacteria during drying and subsequent storage. The most commonly applied approach is drying the bacteria in a protective matrix consisting of components such as carbohydrates, proteins or reconstituted skim milk (RSM) (Broeckx et al., 2016; Huang et al., 2017; Khem et al., 2015; Perdana et al., 2014b). Several mechanisms have been described in literature by which these added components protect cells during drying. These include the water replacement hypothesis and the hydration force explanation. Both state that the presence of small molecules, such as sugars, leads to depression of the membrane phase transition temperature. As a result of this, the membrane integrity is better retained during drying. Also the formation of a glassy matrix upon dehydration aids to the protective effect of matrix components for the embedded bacteria (Bryant et al., 2001; Santivarangkna et al., 2008). From all the components that have been used as drying matrix, the disaccharide trehalose is one of the most investigated protectants. Trehalose is a nonreducing disaccharide consisting of two glucose units having a high glass transition temperature. Moreover, in nature (micro-)organisms also accumulate this sugar to survive under environmental stresses.

\subsection{Intracellular trehalose}

Trehalose is often accumulated intracellularly in organisms capable to survive in extremely dry conditions, such as plants, tardigrades, yeasts and bacteria (Crowe et al., 1992; Schill et al., 2009; Zhang and Yan, 2012). However, not all bacteria can actively accumulate 
intracellular trehalose (Termont et al., 2006). Therefore, it would be an interesting strategy to increase intracellular trehalose concentrations in another way and thereby make bacteria more robust towards drying and subsequent storage. Termont et al. (2006) for example constructed a mutant strain of Lactococcus lactis that allowed accumulation of high concentrations of intracellular trehalose. They observed that this resulted in a higher survival after freeze drying compared to the wild-type. In a similar study by Carvalho et al. (2011), the same approach led to an increased robustness of Lactococcus lactis towards acid and cold shock, though no significant enhancement of freeze drying robustness.

In addition to genetic modification, there are several other methods to increase intracellular trehalose concentrations in cells (Stewart and He, 2019). Several of these methods result in increased permeability of the cellular membrane and thereby facilitate diffusion of extracellular trehalose into the cells. One of these methods that can be used for this purpose is electroporation, or pulsed electric field (PEF) treatment. This approach was successful for loading mammalian cells with trehalose and resulted in increased cell survival upon cryopreservation (Dovgan et al., 2017; Shirakashi et al., 2002). Additionally, in food applications pulsed electric field treatment, aimed to increase intracellular trehalose, resulted in better retention of tissue structure after freezing of spinach or rucola leaves. (Dymek et al., 2014; Phoon et al., 2008). Therefore, this pulsed electric field approach might also be interesting to deliver trehalose or other protective molecules into bacteria. Based on the results obtained for other cell types, we hypothesized that this approach could also be applied to enhance robustness of the bacteria during subsequent processing. Model probiotic Lactobacillus plantarum WCFS1 (Fig. 1.1) is used throughout this study because it is a well-studied lactic acid bacterium; its genome is available, as well as knowledge on its robustness during drying processes (Aryani et al., 2016; Kleerebezem et al., 2003; Perdana et al., 2014b, 2014a; van den Nieuwboer et al., 2016).

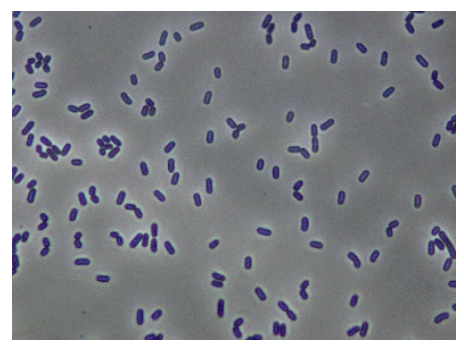

Figure 1.1 Microscopy picture of Lactobacillus plantarum WCFS1 


\subsection{Pulsed electric field}

Pulsed electric field (PEF) employs the application of short high voltage pulses to a conductive (food) product placed between two electrodes (Raso et al., 2016; Wouters et al., 2001a). The applied electric field can lead to pore formation in the cell membrane. This pore formation can be either reversible or irreversible depending on the PEF treatment applied (Fig. 1.2). The effect of PEF on the cell membrane involves an increase in the transmembrane potential difference due to the external electric field. When a critical transmembrane potential difference is reached, pores are formed in the cellular membrane during the application of the pulse. As a result, the cellular membrane can become permeable to ions, sugars, fluorescent probes or larger molecules such as plasmid DNA. After PEF treatment, the pores may reseal under appropriate conditions when the membrane is not damaged too much. In this case membrane electroporation is reversible and the cells are viable if not too many intracellular components are lost. If the cell membrane is damaged too much, for example due to the high electric field applied or nonadequate resealing conditions, the electroporation is irreversible (Fig. 1.2). (Rols and Teissié, 1990; Saulis, 2010)

Before PEF

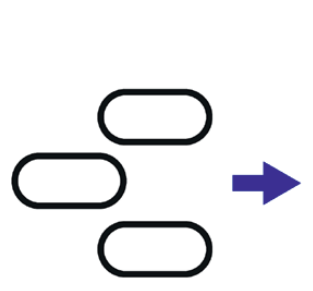

PEF treatment

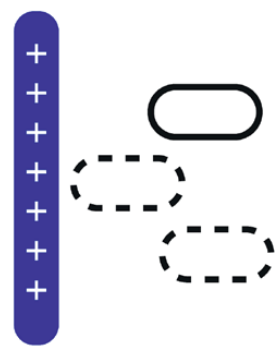

After PEF and resealing

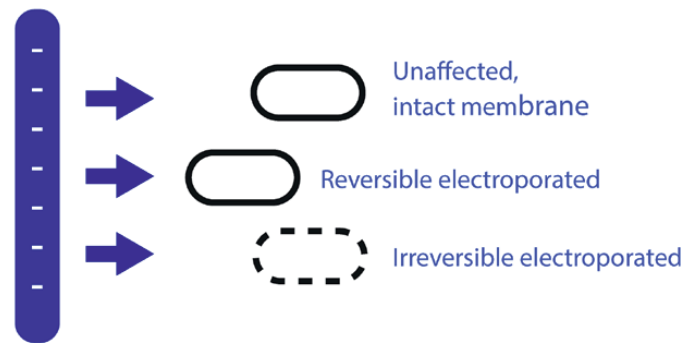

Figure 1.2 Schematic representation of the possible effects of a PEF treatment on cells. Depending on the PEF and resealing conditions cells can be either unaffected, reversible electroporated or irreversible electroporated. Solid lines indicate intact cell membranes and dashed lines permeabilized cell membranes.

Studies on the application of PEF treatment for bacteria mainly focus on irreversible PEF treatments for inactivation purposes. Though, for the application of PEF for increasing intracellular solutes, reversible PEF treatment is essential. Many factors can influence the effect of a PEF treatment on bacteria. These factors can be divided into process parameters, bacterial characteristics and treatment medium characteristics (Table 1.1). 
Table 1.1 Parameters affecting the survival of bacteria after PEF treatment. Adapted from Wouters et al. (2001a) and Timmermans (2018)

\begin{tabular}{lll}
\hline Process parameters & Bacterial characteristics & Treatment medium \\
\hline Electric field strength & Species and strain & Conductivity \\
Pulse duration & Cell size and morphology & $\mathrm{pH}$ \\
Number of pulses & Gram-positive or Gram-negative & Composition \\
Pulse shape & Growth conditions: & Water activity \\
Treatment chamber design & $-\quad$ Growth phase & \\
Temperature & $-\quad$ Temperature & \\
Pulse frequency & - Medium & \\
Specific energy input & - History & \\
& Recovery conditions: & \\
& $-\quad$ Medium & \\
& $-\quad$ Temperature & \\
& - & Recovery time \\
\hline
\end{tabular}

Electric field strength is one of the main parameters affecting the effect of a PEF treatment on cells. The electric field strength can be determined by dividing the applied voltage over the distance between the electrodes. In general higher electric field strengths are required for electroporation of bacteria compared to yeasts, plant cells or mammalian cells due to the smaller cell size of bacteria (Barba et al., 2015; Fox et al., 2006; Heinz et al., 2001). The electric field strength together with the pulse duration, number of pulses and medium conductivity can be used to determine the specific energy input of a PEF treatment (Toepfl et al., 2007). A higher specific energy input often results in more bacterial inactivation, however this is not always the case as at the same energy input a higher electric field strength is more effective (Álvarez et al., 2003). Another important parameter is the temperature; PEF at a higher temperature often results in more bacterial inactivation (Amiali et al., 2007; Cebrián et al., 2016). The effectivity of a specific PEF treatment for bacteria also depends on the bacterial species and strain, large variations between strains were observed in effectiveness of PEF treatments for bacterial inactivation (Timmermans et al., 2014; Toepfl et al., 2007). Overall, many parameters can influence the outcome of a specific PEF treatment. The approach for the PEF treatment in this thesis is therefore based on a combination of the knowledge on the effect of PEF treatment on inactivation of 
bacteria and the knowledge about PEF for molecule uptake in several cell types. For example, a low treatment medium conductivity was found to be beneficial for molecule uptake in combination with cell survival in mammalian cells (Silve et al., 2016).

As mentioned before, PEF treatment has been studied widely for several applications in various cell types. These applications include mild pasteurization, extraction of components from plant or microalgal cells, loading of cells with DNA, i.e. transfection, or small molecules and in the medical world even for cancer treatments (Barba et al., 2015; Miklavčič et al., 2012; Raso et al., 2016). Recently, other researchers used PEF treatment to increase intracellular calcium and magnesium concentrations in Lactobacilli in order to produce ice cream enriched with probiotics and these ions (Góral and Pankiewicz, 2017; Pankiewicz et al., 2020). Larger-scale PEF systems in food industry are readily applied for preservation of fruit juices and in the potato industry for structure softening (Toepfl, 2019). Many other industrial applications of PEF treatments in food industry are currently being developed and marketed (Elea, 2020; Pulsemaster, 2020; Wang et al., 2018). The approach investigated in this thesis may also lead to future applications, for example for spray dried infant formula containing living beneficial bacteria.

\subsection{Objective and outline of this thesis}

The objective of this thesis is to increase survival of lactic acid bacteria during drying by making use of a PEF pre-treatment to facilitate diffusion of molecules across the cell membrane. This PEF pre-treatment is employed to increase intracellular concentrations of protective solutes, such as trehalose (Fig. 1.3). This overall objective is divided into two parts:

1. To develop a PEF treatment resulting in increased intracellular trehalose concentrations, while maintaining a high culture viability.

2. To evaluate whether PEF pre-treatment resulting in increased intracellular trehalose concentrations leads to increased robustness during drying processes and subsequent powder storage. 


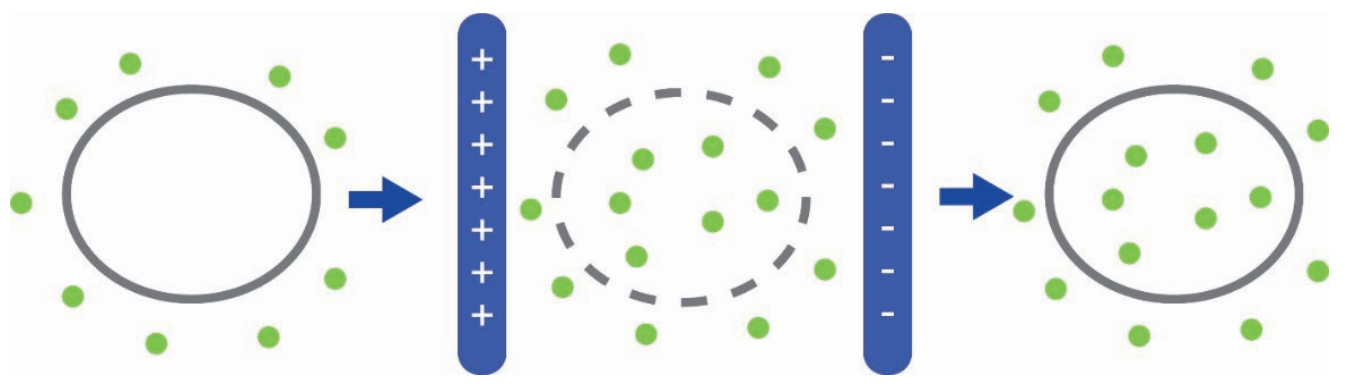

Figure 1.3 Schematic representation of the effect of the PEF treatment aimed for in this thesis. Dashed lines indicate permeabilized cell membranes and solid lines indicate intact cell membranes.

Chapter 2 describes the proof of principle of using pulsed electric field treatment to increase intracellular trehalose concentrations in our model micro-organism Lactobacillus plantarum WCFS1. Survival after PEF treatment and intracellular trehalose concentrations were analysed for PEF treatments with various electric field strengths. Additionally, we link this to the membrane permeability and resealing time after PEF by making use of a fluorescent staining approach.

Chapter 3 continues more in depth on the effect of the PEF treatment on the reversibility of membrane permeabilization in Lactobacillus plantarum WCFS1. This reversibility is essential for the application of PEF for increasing intracellular concentrations. The reversibility of membrane permeabilization was studied with the help of two different fluorescent staining approaches. Additionally, the effect of the PEF treatment parameters electric field strength, pulse duration and number of pulses on membrane permeabilization is discussed.

Subsequently, the PEF pre-treatment was combined with freeze drying and storage tests in chapter 4. To understand the effect of PEF pre-treatment on survival during drying and subsequent storage, the concentrations of intracellular trehalose and lactose in Lactobacillus plantarum WCFS1 during subsequent freeze and spray drying were further investigated.

In chapter 5 we built on the insights gained in chapter four, where it appeared that intracellular trehalose concentrations increased during the drying process when trehalose is present as the drying matrix. We evaluated the effect of different drying matrices on the 
effectivity of a PEF pre-treatment with trehalose in Lactobacillus plantarum WCFS1. Additionally, we investigated intracellular trehalose concentrations in several other lactic acid bacteria to extend the gained insights for Lactobacillus plantarum WCFS1.

The focus of chapter 6 is to further understand the effect of the drying matrices on bacterial survival after spray drying. For this, a single droplet drying approach was used to investigate the effect of various drying matrices on survival of Lactobacillus plantarum WCFS1. More specifically, we investigated whether survival could be related to particle morphology development during single droplet drying.

Chapter 7 provides a general discussion of the results obtained in this thesis. Here, we also describe the effect of several other PEF parameters, which have not been investigated in the previous chapters, that influence the outcome of the PEF treatment. Additionally, it is discussed how the PEF pre-treatment may be scaled- up and implemented into a realistic industrial drying process for drying of bacterial cultures.

Figure 1.4 provides a schematic representation of this thesis outline.

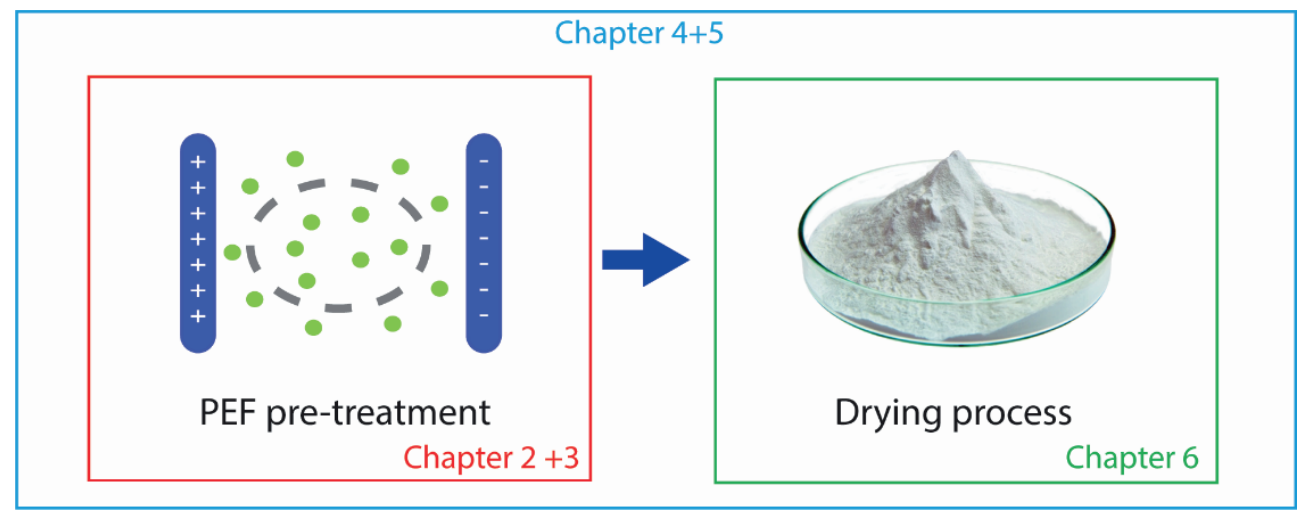

Figure 1.4 Graphical representation of the thesis outline. 



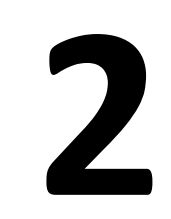

\section{Pulsed electric field for increasing intracellular trehalose content in Lactobacillus plantarum WCFS1}

This chapter has been published as Vaessen, E M J, Besten, H. M. W. Den, Patra, T., Mossevelde, N. T. M. Van, Boom, R. M., \& Schutyser, M. A. I. (2018). Pulsed electric field for increasing intracellular trehalose content in Lactobacillus plantarum WCFS1. Innovative Food Science and Emerging Technologies, 47, 256-261. 


\begin{abstract}
Pulsed electric field processing has been investigated and applied in food industry for several purposes. In this study we propose pulsed electric field processing for increasing the intracellular trehalose content in Lactobacillus plantarum WCFS1, which may enhance bacterial robustness during processing. Our results indicate that it is possible to increase intracellular trehalose content while maintaining high culture viability in Lactobacillus plantarum WCFS1 when applying a PEF treatment with an electric field strength of 7.5 $\mathrm{kV} / \mathrm{cm}$. Fluorescence staining of PEF treated cells with propidium iodide (PI) and SYTO 9 showed that at $7.5 \mathrm{kV} / \mathrm{cm}$ only a small fraction (23\%) of the cells was affected by this PEF treatment, of which approximately half were irreversible affected. Resealing of the pores in the membrane for PI uptake was very fast, maximum in the order of seconds. These results indicate that PEF treatment is promising for increasing intracellular trehalose, but further optimization is required to increase the trehalose content in all cells.
\end{abstract}




\subsection{Introduction}

The application of pulsed electric fields (PEF) is used or considered for use in food processing for pasteurization of fruit juices (Buckow et al., 2013; Timmermans et al., 2014), extraction of components from plant cells (Corrales et al., 2008; Lopez et al., 2009) and tissue softening for easier cutting (Toepfl et al., 2005). PEF treatment induces the formation of pores in the cell membrane, which can be either reversible or irreversible depending on the applied PEFand resealing conditions. The presence of pores in the membrane induces exchange of components with the surroundings of the cells. Diffusion of environmental components into the cells is not (yet) commonly used in food industry. Until now, research to the insertion of components into bacterial cells by electroporation mainly focuses on the introduction of foreign DNA into cells.

In this research we focus on the uptake of small environmental molecules by PEF treatment in bacteria while maintaining the culture viability. This may be important for its robustness during processing. Indeed, it has been shown previously that the insertion of trehalose facilitated by electroporation in mammalian cells led to much better robustness against freezing (Dovgan et al., 2017; Shirakashi et al., 2002). To our knowledge, this method has not yet been used for increasing small molecule concentrations in bacterial cells. Trehalose is one of the protective small molecules that could enhance robustness during processing (Leslie et al., 1995; Termont et al., 2006). Therefore this study investigates the increase of intracellular trehalose content in Lactobacillus plantarum WCFS1 through electroporation while maintaining the culture viability.

Lactobacillus plantarum WCFS1 is a commonly studied model microorganism for the production of probiotic formulations (Perdana et al., 2014a, 2013). Probiotics are defined as live microorganisms that, when administered in adequate amounts, confer a health benefit on the host (Hill et al., 2014). Probiotics can be supplied to the consumer in dried formulation to enhance product shelf-life, which requires processing steps such as spray or freeze drying. Survival of the microorganisms during these processing steps is essential for their beneficial function in the human gastrointestinal tract. However, especially for spray drying, their survival is relatively low, which is a big challenge in the production process (Meng et al., 2008). There are several methods to improve the survival of bacteria during these drying procedures, for example by encapsulation of the microorganisms. Another, less intensively studied approach for protection is intracellular protection. Intracellular 
trehalose could enhance survival during these processes (Termont et al., 2006). One way of increasing intracellular trehalose content could be electroporation in a solution of trehalose.

Electroporation is used commonly in biotechnology for inserting plasmid DNA into bacterial cells (transformation). For transformation, cells should be reversibly permeabilized to take up the plasmid and survive. The main difference between plasmid uptake and our aim is the size of the molecules and thus the size of the pores in the membrane required for the uptake of the molecules. Trehalose is a much smaller molecule than plasmid DNA and thus could be more facile to introduce in the cell by electroporation (Saulis, 2010). It is essential that the poration is reversible, and that the inactivation of the bacteria during the PEF treatment is as limited as possible.

There are many PEF process parameters that influence the effects of a PEF treatment such as the electric field strength, pulse shape, pulse width, pulse frequency etc. (Raso et al., 2016). Besides the PEF process parameters, the treatment medium is an important factor for reversible or irreversible pore formation by PEF treatment. One of the main influencing parameters is the medium conductivity. Silve et al. (Silve et al., 2016) found that a low medium conductivity $(0.1 \mathrm{~S} / \mathrm{m})$ was more effective for reversible permeabilization of mammalian cells than a high medium conductivity $(1.5 \mathrm{~S} / \mathrm{m})$. After the PEF treatment the cells need to reseal their membrane to maintain viability, and therefore the period after the treatment, referred to as resealing period, is considered an important parameter that can be influenced by the temperature (Teissie et al., 2005). In our study we will vary the electric field strength to find optimal conditions to increase the intracellular trehalose content while maintaining cell viability. Furthermore, staining with propidium iodide will be used to study the reversibility of the pore formation.

\subsection{Materials and methods}

\subsubsection{Microorganism and pre-culture conditions}

Fresh cultures of Lactobacillus plantarum WCFS1 were obtained by plating from frozen stocks on De Man Rogosa and Sharpe (MRS) agar plates (MRS: Merck, Germany, Bacteriological agar: Oxoid, United Kingdom). The plates were incubated at $30^{\circ} \mathrm{C}$ for $60-70$ hours under micro aerobic conditions in jars containing 6\% oxygen (Anoxomat, Mart Microbiology, the Netherlands). After incubation the plates were stored at $4^{\circ} \mathrm{C}$ until further 
use for a maximum of 3 days. For every experiment a culture was prepared by transferring a single colony into $10 \mathrm{~mL}$ MRS broth (Merck, Germany), growing for $24 \pm 2$ hours in $30^{\circ} \mathrm{C}$, followed by a 1:100 dilution in $10 \mathrm{~mL}$ MRS broth and growing overnight (16-18 hours) at $30^{\circ} \mathrm{C}$ before starting the PEF experiment.

\subsubsection{Culture preparation for PEF experiments}

Five $\mathrm{mL}$ of an overnight culture was centrifuged (Thermo-Fischer Scientific, USA) at 13,500 $\times g$ for 10 minutes at room temperature. The $\mathrm{pH}$ of the supernatant was measured (Toledo Inlab Expert, Switzerland) and was $3.9 \pm 0.1$ for all experiments. The resulting pellet was washed once with $5 \mathrm{~mL}$ washing solution (Table 2.1) and after centrifugation dissolved in 5 $\mathrm{mL}$ PEF medium (Table 2.1). This resulted in a culture in PEF medium containing approximately $3-4 \cdot 10^{9}$ cells $/ \mathrm{mL}$.

\subsubsection{PEF equipment and settings}

PEF treatment was performed in disposable electroporation cuvettes with an electrode distance of $2 \mathrm{~mm}$ (Bio-Rad, USA) using the Gene-Pulser Xcell equipment, including the PC module (Bio-Rad, USA). The PEF settings were as follows: for the voltage in the electric field screening experiments 500,1000,1500, 2000 and $2500 \mathrm{~V}$ were used, with 0.1 ms pulse duration, 2 pulses, pulse interval $5 \mathrm{~s}$ as this was the minimum setting of the equipment, and square wave pulses. The specific energy input of these PEF treatments is presented in table 2.2. Other experiments were only performed at $1500 \mathrm{~V}$ with the same other settings. Droop values (average decay of the pulse height) were $5-6 \%$ for all experiments. 
Table 2.1 Composition, $\mathrm{pH}$ and conductivity of solutions used in the PEF experiments. All solutions were autoclaved for 15 minutes at $121^{\circ} \mathrm{C}$ before use.

\begin{tabular}{|c|c|c|c|}
\hline & Washing solution & PEF medium ${ }^{1}$ & PBS \\
\hline \multirow[t]{7}{*}{ Composition } & $0.29 \mathrm{~g} \mathrm{NaCl}^{\mathrm{b}}$ & 99.3 g Trehalose $^{\mathrm{a}}$ & $1.93 \mathrm{~g} \mathrm{Na}_{2} \mathrm{HPO}_{4} \cdot 2 \mathrm{H}_{2} \mathrm{O}^{\mathrm{a}}$ \\
\hline & $0.89 \mathrm{~g}$ & $0.29 \mathrm{~g} \mathrm{NaCl}^{\mathrm{b}}$ & $0.35 \mathrm{~g} \mathrm{NaH}_{2} \mathrm{PO}_{4} \cdot \mathrm{H}_{2} \mathrm{O}^{\mathrm{a}}$ \\
\hline & $\mathrm{Na}_{2} \mathrm{HPO}_{4} \cdot 2 \mathrm{H}_{2} \mathrm{O}^{\mathrm{a}}$ & $0.89 \mathrm{~g}$ & $8.20 \mathrm{~g} \mathrm{NaCl}^{\mathrm{b}}$ \\
\hline & $0.69 \mathrm{~g} \mathrm{NaH}_{2} \mathrm{PO}_{4} \cdot \mathrm{H}_{2} \mathrm{O}^{\mathrm{a}}$ & $\mathrm{Na}_{2} \mathrm{HPO}_{4} \cdot 2 \mathrm{H}_{2} \mathrm{O}^{\mathrm{a}}$ & \multirow{4}{*}{$\begin{array}{l}1000 \text { g demineralized } \\
\text { water }\end{array}$} \\
\hline & $0.095 \mathrm{~g} \mathrm{MgCl}_{2} \cdot 6 \mathrm{H}_{2} \mathrm{O}^{\mathrm{b}}$ & $0.69 \mathrm{~g} \mathrm{NaH}_{2} \mathrm{PO}_{4} \cdot \mathrm{H}_{2} \mathrm{O}^{\mathrm{a}}$ & \\
\hline & $1000 \mathrm{~g}$ demineralized & $0.095 \mathrm{~g} \mathrm{MgCl}_{2} \cdot 6 \mathrm{H}_{2} \mathrm{O}^{\mathrm{b}}$ & \\
\hline & water & $\begin{array}{l}1000 \mathrm{~g} \\
\text { demineralized water }\end{array}$ & \\
\hline $\mathrm{pH}$ & 6.9 & 6.8 & 7.2 \\
\hline Conductivity & $0.21 \mathrm{~s} / \mathrm{m}$ & $\begin{array}{l}0.15 \mathrm{~S} / \mathrm{m} \text { (with } \\
\text { bacteria) }\end{array}$ & n.a. \\
\hline
\end{tabular}

${ }^{1}$ Adapted from Silve et al. (2016) ; salts from SNM medium, with addition of trehalose

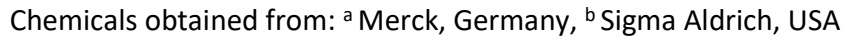

\subsubsection{Electric Field screening experiments}

From each culture in PEF medium, one part was taken aside as a control sample, for which all steps were similar except for skipping the PEF treatment. Per experiment, two electroporation cuvettes were filled with $400 \mu \mathrm{L}$ culture in PEF medium and electroporated using the described equipment and settings. After the PEF treatment, the content of these two cuvettes was immediately pooled into one $1.5 \mathrm{~mL}$ vial to have enough sample volume for further analysis. The electroporated culture was left for resealing at room temperature $\left( \pm 21^{\circ} \mathrm{C}\right)$ for 30 minutes. After these 30 minutes, samples were taken for survival assessment and intracellular trehalose content measurements. 
Table 2.2 Specific energy of the PEF treatments with 2 pulses of $100 \mu$ s pulse duration at various electric field strengths.

\begin{tabular}{ll}
\hline $\begin{array}{l}\text { Electric field } \\
\text { strength } \\
(\mathbf{k V} / \mathrm{cm})\end{array}$ & $\begin{array}{l}\text { Specific energy input } \\
\mathbf{1}^{\mathbf{1}}\end{array}$ \\
\hline $\mathbf{2 . 5} / \mathbf{k g})$ \\
$\mathbf{5 . 0}$ & 1.8 \\
$\mathbf{7 . 5}$ & 7.2 \\
$\mathbf{1 0 . 0}$ & 16.2 \\
$\mathbf{1 2 . 5}$ & 28.9 \\
\hline
\end{tabular}

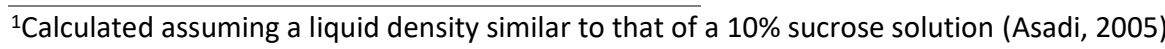

\subsubsection{Survival assessment}

Bacterial survival after the PEF treatment was based on plate counting. For each sample (control and electroporated) three times $50 \mu \mathrm{L}$ of sample was dissolved in $450 \mu \mathrm{L}$ phosphate-buffered saline (PBS, Table 2.1) and subsequently decimally diluted until $10^{-6}$, and this dilution was plated on MRS agar plates in duplicate, resulting in 6 plates in total per sample. Plates were incubated for $48-96$ hours at $30^{\circ} \mathrm{C}$ under micro aerobic conditions. After incubation colony forming units (cfu) per plate were counted of plates containing between 30 and 300 colonies. The average of the 6 plates per sample was taken for calculation of the survival. Survival was calculated by dividing the $\mathrm{cfu} / \mathrm{mL}$ of electroporated samples over the $\mathrm{cfu} / \mathrm{ml}$ of the control sample.

\subsubsection{Intracellular trehalose content measurements}

After the 30 minutes of resealing time the control and PEF treated samples were centrifuged for 10 minutes at $13,500 \times g$ and $4{ }^{\circ} \mathrm{C}$. The resulting pellet was washed three times with 1 $\mathrm{mL}$ PBS to remove all extracellular trehalose. The supernatant of the third washing step was stored at $-20^{\circ} \mathrm{C}$ for HPLC analysis to check the washing efficiency. After washing, the resulting pellet was dissolved in $0.75 \mathrm{~mL}$ of milliQ water and transferred into bead beater vials containing $0.1 \mathrm{~mm}$ silica beads (MP Biomedicals, USA). The samples were bead beated 5 times 1 minute at $4 \mathrm{~m} / \mathrm{s}$ (MP Biomedicals, USA). In between bead beating steps samples were kept on ice for 1-3 minutes to prevent too much heating of the samples. After bead beating the samples were centrifuged again ( 10 minutes, $13,500 \times g, 4^{\circ} \mathrm{C}$ ) and subsequently the supernatant was transferred to a $1.5 \mathrm{~mL}$ vial and stored in $-20^{\circ} \mathrm{C}$ until HPLC analysis. 
HPLC analysis was performed using a Rezex RSO-Oligosaccharide column (Phenomex, USA) at $80^{\circ} \mathrm{C}$ with milliQ water as a mobile phase in a flow rate of $0.3 \mathrm{~mL} / \mathrm{min}$ in combination with a RI detector (Shodex RI-201, Japan).

\subsubsection{Fluorescent staining experiments}

The evaluation of the number of cells that were (ir)reversibly permeabilized during PEF treatment, was done by using fluorescent staining. Two fluorescent stains for membrane integrity were used together in these experiments; SYTO 9 and propidium iodide (PI) (LIVE/DEAD kit, Invitrogen, USA). PI is a membrane impermeable stain which can only enter cells with a damaged membrane and a strong red fluorescence signal can be visualized by fluorescence microscopy upon binding of PI to nucleic acids. SYTO 9 is a membrane permeable stain which colours all cells green. The bacteria were stained either before the PEF treatment and 30 minutes after the PEF treatment (section 2.7.1), or at different time points after the PEF treatment to follow the resealing of the pores in time (section 2.7.2).

\subsubsection{Addition of stains before and after PEF treatment}

Staining the cells before the PEF treatment was done by the addition of PI and SYTO 9 to 1 $\mathrm{mL}$ of the culture in PEF medium, resulting in final stain concentrations of $40 \mu \mathrm{M} \mathrm{PI}$ and 3.3 $\mu \mathrm{M}$ SYTO 9. Subsequently, one part was taken aside as a control for initial membrane permeability (without PEF treatment) and the other part $(400 \mu \mathrm{L})$ was pipetted into an electroporation cuvette for PEF treatment, performed as described before (section 2.3). After the PEF treatment, the PEF treated and the control samples were kept in a dark environment for approximately 10-20 minutes before imaging using fluorescence microscopy (Axioskop 40FL Carl Zeiss, Germany). From the same biological sample, a second cuvette was PEF treated without addition of any stains, after which the culture was transferred to a $1.5 \mathrm{~mL}$ vial and left for 30 minutes of resealing. After these 30 minutes of resealing $100 \mu \mathrm{L}$ of the sample were mixed with $200 \mu \mathrm{L}$ stain solution. The stain solution was made by dissolving PI and SYTO 9 in PBS in final concentrations of respectively $40 \mu \mathrm{M}$ and 3.3 $\mu \mathrm{M}$. This mixture was left for 10-20 minutes in the dark before fluorescence microscopic analysis. Approximately 20 images were captured per sample at a magnification of 630x using an Olympus XC30 camera (Olympus, Japan) and CellSens imaging software (Olympus, Japan). Image analysis was performed using a Matlab script to discriminate between red and green cells, as was described earlier by Perdana et al. (2012). 


\subsubsection{Addition of stains at different time points after PEF to follow membrane resealing} To analyse the resealing of the membrane in time after PEF treatment, stains were added to the PEF treated bacteria at different time points after the PEF treatment. Again a staining solution was made by adding PI and SYTO 9 in PBS in final concentrations of respectively 40 $\mu \mathrm{M}$ and $3.3 \mu \mathrm{M}$. Immediately when the PEF treatment was finished, a stopwatch was set and at several time points (approximately at 10, 20, 30, 60 seconds, 5 and 30 minutes) the stains were added. These samples were left for 10-20 minutes in darkness before fluorescence microscopic analysis as described in section 2.2.7.1.

\subsection{Experimental set-up and statistical analysis}

All experiments were carried out at least three times with different biologically independent samples, obtained from different pre-cultures of L. plantarum WCFS1. The results were averaged or presented as single data points in the graphs. The significance was tested with a Student's $t$-test, using a $P$-value of 0.05 .

\subsection{Results and discussion}

\subsubsection{PEF treatment for increasing intracellular trehalose content}

Electric field screening experiments were performed to find out whether it is possible to increase the intracellular trehalose content in Lactobacillus plantarum WCFS1 while maintaining culture viability. Electric field strengths from 2.5 to $12.5 \mathrm{kV} / \mathrm{cm}$ were investigated by applying two square wave pulses of $100 \mu \mathrm{s}$. The field strengths used in this experiment were higher than commonly used field strengths for similar experiments in mammalian cells (Shirakashi et al., 2002; Silve et al., 2016) because bacteria are much smaller than mammalian cells; therefore a higher field strength is required to affect the bacteria with PEF (Saulis, 2010). However, to maintain the viability, the field strengths that we used were lower than what is used to inactivate bacteria, which is generally above 15 $\mathrm{kV} / \mathrm{cm}$ (Barba et al., 2015). Usually the temperature increase during the PEF process is more when a higher field strength is applied, which may lead to additional inactivation during the PEF treatment. In our case this effect was very small (maximum increase of 4 으 12.5 $\mathrm{kV} / \mathrm{cm})$, because of the low conductivity of the solution $(0.15 \mathrm{~S} / \mathrm{m})$ in combination with the relatively low electric field strengths. 

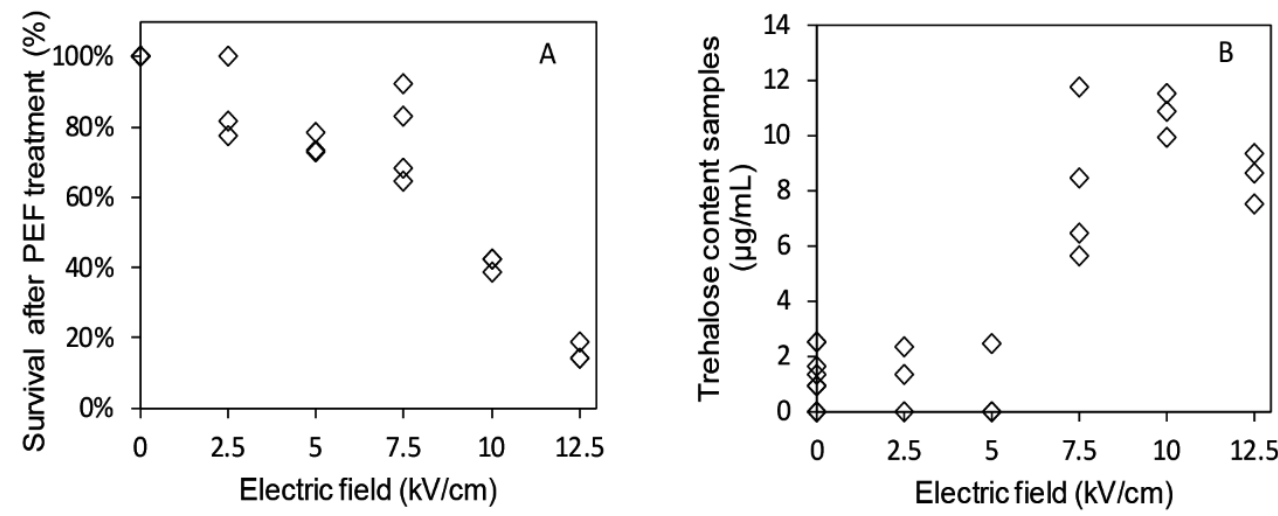

Figure 2.1 Survival (A) and intracellular trehalose content (B) after PEF treatment at various electric field strengths $(2.5-12.5 \mathrm{kV} / \mathrm{cm})$. A: Survival is based on plate counts of the PEF treated samples versus the control samples. B: Measurements at $0 \mathrm{kV} / \mathrm{cm}$ are the control (not PEF treated) samples. Data points at $0 \mu \mathrm{g} / \mathrm{mL}$ were measured but below the detection limit of the HPLC method ( $\sim 1 \mu \mathrm{g} / \mathrm{ml}$ trehalose). Each data point at a certain electric field strength represents one biological replicate. At least three biological replicates were measured for each electric field strength.

The results show that an increasing electric field strength led to a decrease in survival after PEF treatment (Fig. 2.1A). At field strengths of 10 and $12.5 \mathrm{kV} / \mathrm{cm}$ the survival was below $50 \%$. At the same time, a higher intracellular trehalose concentration after PEF was observed at 7.5, 10 and $12.5 \mathrm{kV} / \mathrm{cm}$ (Fig. 2.1B). This indicates that it is possible to use PEF for increasing the intracellular trehalose concentration while limiting the microbial inactivation. At $7.5 \mathrm{kV} / \mathrm{cm}$ there was both an enhanced intracellular trehalose content and a high survival after the PEF treatment (62-93\%). A critical electric field strength was observed between 5 and $7.5 \mathrm{kV} / \mathrm{cm}$ for trehalose diffusion into the cell. At 2.5 and $5 \mathrm{kV} / \mathrm{cm}$, no increase was observed in intracellular trehalose compared to the control $(0 \mathrm{kV} / \mathrm{cm})$ samples. Note that the trehalose content of the cells is presented in $\mu \mathrm{g} / \mathrm{mL}$ sample. The trehalose concentrations in the samples at 7.5, 10 and $12.5 \mathrm{kV} / \mathrm{cm}$ were in a similar range, while the survival after PEF decreased with increasing field strength from 7.5 to $12.5 \mathrm{kV} / \mathrm{cm}$. When calculating the intracellular trehalose concentration per viable cell, an increase in intracellular trehalose concentration was found from 7.5 to $12.5 \mathrm{kV} / \mathrm{cm}$. However, the intracellular trehalose could be in all cells, or may be inserted at high concentrations in only a small portion of these cells. It is known that PEF treatment does not affect all cells in the medium in the same way, which can be due to their orientation in the medium, to shielding 
of the electric field by other cells present and/or variations in the membrane or biological state of the individual bacteria (Pucihar et al., 2007; Toepfl et al., 2007).

\subsubsection{Propidium iodide staining to study solute uptake during PEF treatment}

To find out how many cells were affected by the PEF treatment, propidium iodide was used as a marker molecule. Experiments were performed at $7.5 \mathrm{kV} / \mathrm{cm}$, because this electric field strength resulted in an increase in intracellular trehalose content while the culture viability was still high. Other pulse parameters were the same as for the electric field screening experiments.

Only a small part, approximately $23 \%$, of the bacterial population exhibited a permeable membrane for PI using these PEF conditions (Fig. 2.2). Because when PI and SYTO 9 were added to the culture in PEF medium before the PEF treatment, only $23 \%$ of the cells was stained red after the PEF treatment. This indicates that $77 \%$ of the cells may not be affected by the PEF treatment (Fig. 2.2). From the fraction of cells that was affected, approximately half had a reversibly permeabilized membrane (approximately 13\%). This fraction was the difference in red $(\mathrm{PI})$ stained cells between addition of the stains before the PEF treatment and 30 minutes afterwards. This may imply that the trehalose concentration that was measured before in fact might originate from a high trehalose concentration achieved in a limited fraction of the bacterial population, while other cells may not have been affected. 


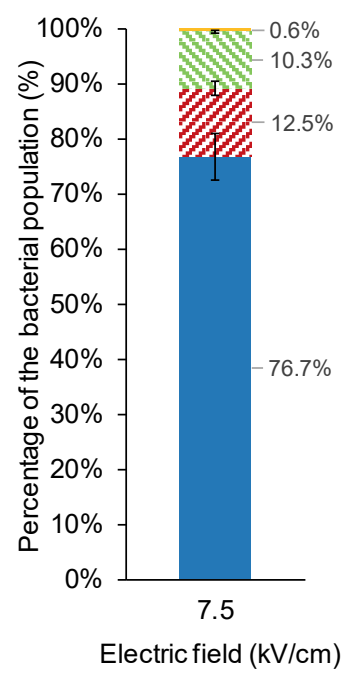

Figure 2.2 Membrane permeability due to PEF treatment measured using PI staining. PEF treatment was performed with 2 pulses of $100 \mu \mathrm{s}$ at $7.5 \mathrm{kV} / \mathrm{cm}$. Percentage of the population with a permeable membrane for PI before PEF treatment ( 1 ), an irreversibly permeable membrane 30 minutes after the PEF treatment (\$), a reversibly permeable membrane 30 minutes after PEF treatment (") and without membrane permeability for PI during PEF treatment (घ). Error bars indicate standard deviations of 3 biological replicates.

The other $10 \%$ had an irreversibly damaged membrane, as these cells were stained red when the stains were added 30 minutes after the PEF treatment. This fraction may well be considered to be dead. However, comparing membrane damage with bacterial survival can be complicated because of the different viability states of bacteria (culturable/metabolically active/intact membrane) (Davis, 2014; Sträuber and Müller, 2010). Garcia et al. (2007) found a good correlation between $L$. plantarum cells that were stained with PI after PEF treatment and cells that were not culturable anymore. Ulmer et al. (2002) found however no significant decrease in plate counts at electric field strengths below $19 \mathrm{kV} / \mathrm{cm}$ with energy inputs until $42 \mathrm{~kJ} / \mathrm{kg}$, but did find an increase in membrane permeability at these field strengths. The energy input and electric field strength in this study $(16.2 \mathrm{~kJ} / \mathrm{kg}$ at 7.5 $\mathrm{kV} / \mathrm{cm}$ ) were lower, but resulted in a slight decrease in plate counts and permeability (Fig. 2.1 \& 2.2). Variations between studies may be due to differences in PEF settings or other factors such are treatment medium composition, but also due to the presentation of the results (log-scale versus percentage) of the plating method. 
Assuming that the increase in intracellular trehalose concentration that we measured with HPLC analysis (Fig. 2.1) is in the bacteria that were reversible permeabilized for PI, the amount of intracellular trehalose in these cells can be calculated from the results of section 3.1 using equation 2.1 .

$$
C_{\text {tre }}^{i}=\frac{C_{\text {tre }}^{s}}{N_{\text {cells }} \cdot x_{\text {rev }} \cdot V_{\text {cell }} \cdot x_{\text {cytosol }}}
$$

In which $C_{t r e}^{i}$ is the intracellular trehalose concentration in the reversible permeabilized cells in $\mathrm{mM}, C_{\text {tre }}^{s}$ the trehalose concentration in the sample in $\mathrm{mM}$, which can be obtained from Fig. 2.1 by dividing by the molecular weight of trehalose, $N_{\text {cells }}$ the total number of cells per $\mathrm{mL}$ sample, $x_{\text {rev }}$ the reversible permeabilized fraction, $V_{\text {cell }}$ the bacterial cell volume and $x_{\text {cytosol }}$ the fraction cytosol of the total cell volume.

The cell volume of $L$. plantarum WCFS1 was estimated, based on cell size measurements from microscopic pictures, to be around $9.2 \cdot 10^{-13} \mathrm{~mL}$, which is in a similar range of what was found in literature (Dumont et al., 2004). The total number of cells was approximately $3.5-4 \cdot 10^{9}$ cells per $\mathrm{mL}$ based on plate counting results. By assuming $x_{\text {rev }}$ to be 0.1 (Fig. $2.2), x_{\text {cytosol }}$ to be 0.7 (Luby-Phelps, 1999), and taking a trehalose content in the sample of $8 \mu \mathrm{g} / \mathrm{mL}$ from figure 2.1 , the possible amount of trehalose in these cells is calculated to be approximately $100 \mathrm{mM}$, or around $35 \%$ of the concentration that was imposed from the outside during PEF treatment. This amount is similar to what was earlier estimated for trehalose uptake during PEF treatment in mammalian cells (Shirakashi et al., 2002). Termont et al. (2006) found that intracellular trehalose concentrations of approximately $30-50 \mathrm{mg} / \mathrm{g}$ wet cell weight (wcw) in Lactococcus lactis protected the bacteria during freeze drying and enhanced resistance against bile salts and gastric acid. By assuming a wet cell weight of approximately $1 \mathrm{pg}$ per cell (the biggest part of the cell consists of water), our method resulted in an intracellular trehalose content of approximately $25 \mathrm{mg} / \mathrm{g} \mathrm{wcw}$, which is slightly below the concentrations of Termont et al. (2006) that were obtained via genetic modification.

Of course, when making these estimations based on PI uptake, we need to take into account that trehalose is another molecule than PI; trehalose has a molecular weight of 342 Da while PI has a molecular weight of $668 \mathrm{Da}$. Saulis (2010) described that molecular uptake by PEF 
treatment can be different for molecules of different sizes, e.g. small ions compared to mannitol and sucrose. This strongly depends on the pore size, which can be affected by several pulse parameters such as electric field strength and pulse width. Also after the pulse, during the resealing phase, a pore may stay open longer for small molecules such as ions than for molecules like sugars and PI (Saulis, 2010).

To study the time that the pores stayed open for PI uptake after PEF treatment, PI was added at different time points after the PEF treatment. The results indicate that the pores created during the PEF treatment, close or reduce in size really fast after the treatment, i.e. in the range of maximum several seconds (Fig. 2.3). This is much shorter than what Shirakashi et al. (2004) described for mammalian cells: they found resealing times for PI uptake in the order of several minutes. All the samples that were stained before the PEF treatment ( $\mathrm{t}=0$ in Fig. 3 ) showed significantly higher PI uptake than the samples stained at different time points after PEF treatment. This indicates that for L. plantarum WCFS1, resealing was very fast after the PEF treatment.

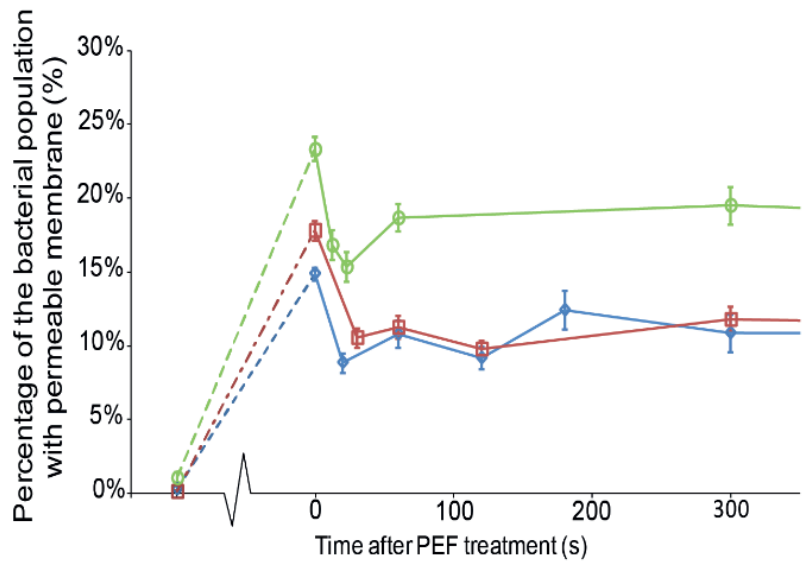

Figure 2.3 Cell membrane permeability for PI before, during and after PEF treatment over time. Time points on the $x$-axis represent the moment at which the stain was added to the culture. $t=0 \mathrm{~s}$ is the moment of the PEF treatment. The first data point (before the axis break) is the control (without/before PEF) and the data points at $t=0$ s are with stains added before PEF treatment. Data points after $t=0$ indicate the membrane permeability at the specific time at which the stains were added. One data point at $t=1800$ or $2400 \mathrm{~s}$ for each replicate is not presented, this data point was similar to the $t=300 \mathrm{~s}$ data point. Each symbol represents one biological replicate, error bars indicate the standard error of the mean of different pictures, $n=10-20$ 
Given the relatively small fraction of the bacterial population currently affected by the PEF treatment, it is important to increase the reversibly electroporated fraction of the bacterial population for future applications of this process in food industry. A bigger fraction of reversible electroporated cells, containing trehalose, is required for the potential beneficial effect during processing. For this beneficial effect trehalose should be present in the cells for internal protection of the membrane and other molecules such as proteins in the cytosol. To further develop the proposed PEF method, it should be evaluated to other bacteria as well. However, the optimal PEF conditions for trehalose transfer probably differ between bacterial species or even strains as was demonstrated before for inactivation of bacteria (Saldaña et al., 2009). Moreover, further studies are required to critically test the benefits of an additional PEF treatment to improve survival during subsequent processing.

\subsection{Conclusions}

Pulsed electric field treatment can increase the intracellular trehalose content of Lactobacillus plantarum WCFS1 while maintaining culture viability. Electric field screening experiments with two pulses of $100 \mu$ s indicated a critical electric field strength for trehalose diffusion into the cell between 5 and $7.5 \mathrm{kV} / \mathrm{cm}$. Two square wave pulses of $100 \mu$ s at 7.5 $\mathrm{kV} / \mathrm{cm}$ led to an increase in intracellular trehalose while maintaining cell viability after the PEF treatment. Study of the membrane permeability during and after this PEF treatment showed that only a small fraction of the bacterial population was reversible affected by the current PEF treatment, but these cells acquired a high internal concentration of trehalose. The pores in the cell membrane of L. plantarum WCFS1 closed very fast after the PEF treatment for the uptake of $\mathrm{PI}$, which indicates that resealing time for PI uptake is in the order of seconds. Optimization of trehalose uptake by PEF treatment should focus on a higher fraction of reversible electroporated cells, containing trehalose, in order to potentially enhance bacterial robustness during processing.

\section{Acknowledgements}

This work took place within the framework of the Institute of Sustainable Process Technology (ISPT), The Netherlands. The authors would like to thank Maurice Strubel for his help with the HPLC measurements. 



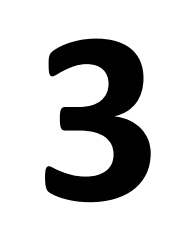

\section{Reversibility of membrane permeabilization upon pulsed electric field treatment in Lactobacillus plantarum WCFS1}

This chapter has been published as Vaessen, E. M.J., Timmermans, R. A. H., Tempelaars, M. H., Schutyser, M. A. I., \& den Besten, H. M. W. (2019). Reversbility of membrane permeabilization in Lactobacillus plantarum WCFS1 after pulsed electric field treatment. Scientific Reports, 19990. 


\begin{abstract}
Pulsed electric field (PEF) treatment, or electroporation, can be used to load molecules into cells. The permeabilizing effect of the PEF treatment on the cellular membrane can be either reversible or irreversible depending on the severity of the PEF treatment conditions. The influence of PEF on the reversibility of membrane permeabilization in Lactobacillus plantarum WCFS1 by two different fluorescent staining methods was investigated in this study. Whereas staining with propidium iodide (PI) before and after PEF treatment indicated small reversible permeabilized fractions of maximum $14 \%$, the use of a double staining method with PI and SYTOX Green suggested larger reversible permeabilized fractions up to $40 \%$ of the population. This difference shows that the choice for a fluorescent staining method affects the conclusions drawn regarding reversibility of membrane permeabilization. Additionally, the effect of PEF treatment conditions on membrane integrity was compared, indicating a relation between critical electric field strength, cell size and membrane permeabilization. Overall this study showed the possibilities and limitations of fluorescent membrane integrity staining methods for PEF studies.
\end{abstract}




\subsection{Introduction}

Electroporation, or pulsed electric field (PEF) treatment, is currently widely used for many applications in several cell types, including bacteria, microalgae, mammalian cells and plant cells or tissue (Shi et al., 2018; Wang et al., 2018). The reason can be to inactivate cells, to extract components from cells or to load cells with components such as DNA or small molecules. All these applications employ the permeabilizing effect of the electric field on the cell membrane. A PEF treatment is applied to the cells in a conductive medium between two electrodes using one or more high voltage pulses with a pulse duration in the nanosecond to millisecond range (Raso et al., 2016). The electric field strength applied between the electrodes influences the transmembrane potential difference over the cellular membrane. When this potential difference exceeds a critical value, pore formation occurs in the membrane of the cells (Kotnik et al., 2010; Teissié and Rols, 1993). Depending on the treatment conditions, pore formation in the cell membrane can be either reversible or irreversible. Loading of cells with DNA or small molecules requires reversible electroporation and survival of the electroporated cells, whereas inactivation requires irreversible electroporation leading to cell death (Toepfl et al., 2005).

Loading of small molecules, such as trehalose, into cells has been shown to increase the processing robustness of mammalian cells and plant tissue (Chen et al., 2001; Phoon et al., 2008). Our previous study demonstrated that PEF treatment can also be used to increase intracellular trehalose in the model probiotic bacterium, Lactobacillus plantarum WCFS1, while maintaining culture viability (Vaessen et al., 2018). However, for enhanced robustness during further processing of this probiotic bacterium, the PEF treatment should be further optimized to increase the fraction of reversible electroporated cells loaded with trehalose (Vaessen et al., 2019). In order to achieve this, more knowledge is required related to PEF processing parameters and their effect on reversibility of membrane permeabilization.

A common method to detect bacterial membrane permeability is staining of cells with propidium iodide (PI). $\mathrm{PI}$ is a small $(668 \mathrm{Da}$ ) hydrophilic fluorescent probe that can enter the cell only when the membrane integrity is compromised, for example because of pore formation during PEF treatment (García et al., 2007). When it binds to nucleic acids inside the cell, the red fluorescence of $\mathrm{PI}$ increases 20 to $30 \mathrm{fold}$, making it a valuable tool to detect membrane permeability and in some cases cell viability (García et al., 2007). Many studies used this method to detect irreversible pore formation in bacteria by addition of the 
fluorescent stain after the PEF treatment (Aronsson et al., 2005; Tryfona and Bustard, 2008; Unal et al., 2002; Wouters et al., 2001b). Furthermore, some studies have used it to detect reversibility of pore formation in both Gram-positive and Gram-negative bacteria to study inactivation mechanisms of the PEF treatment (García et al., 2007; Ulmer et al., 2002). In these studies, PI was added before and after PEF treatment to study membrane permeabilization in relation to loss of viability for inactivation purposes.

In addition to PI staining, also other fluorescent stains have been used to study the effect of electroporation on microbial cells. One of these stains is cFDA (carboxyfluorescein diacetate), which is a probe for esterase activity and is therefore used as an indication for cell viability after PEF (Jaeger et al., 2009). Furthermore, respiratory activity is sometimes employed as a measure of cell viability by using fluorescent redox probes and has been studied in relation to PEF as well (Yaqub et al., 2004). The results obtained with different stains cannot always be compared as they can focus on other aspects of cell viability (Breeuwer and Abee, 2000; Sträuber and Müller, 2010). Therefore, we use in this study a second stain with a similar function as PI, namely SYTOX Green. SYTOX Green is also a membrane integrity stain, which binds nucleic acids after entering a cell and has been used to study membrane permeability after PEF treatment as well (Bodénès et al., 2019; Garcia et al., 2016). A recent study also showed that not only the cellular membrane is affected by PEF, but also the cell wall material (Pillet et al., 2016). This cell wall itself however, consisting of peptidoglycan layers, is not expected to be a barrier for molecules with the size of PI or SYTOX Green (Demchick and Koch, 1996).

The aim of this study is to understand the reversible permeabilization of the cellular membrane upon PEF treatment for the uptake of molecules in Lactobacillus plantarum WCFS1. To study the reversibility of membrane permeabilization two different methods were used; firstly the addition of PI before and after PEF treatment and secondly, a combination staining of PI and SYTOX Green. The first method has the advantage of using only one membrane permeability probe, with the disadvantage that two parallel PEF treatments are required. For the second method this was the other way around, the advantage is that only one PEF treatment is required and more analysis options are possible on the different fractions using flow cytometry. The disadvantage of the second method is that two different markers for membrane permeability are used. These two staining 
methods are compared for different PEF treatments, and in addition the effect of the different PEF treatments on membrane permeability is discussed.

\subsection{Materials and methods}

\subsubsection{Culture preparation for electroporation experiments}

Lactobacillus plantarum WCFS1, originally isolated from human saliva (Kleerebezem et al., 2003), was obtained from the Food Microbiology strain collection. For every PEF experiment a fresh stationary phase culture of Lactobacillus plantarum WCFS1 was used. L. plantarum WCFS1 was cultured in the same way as described in previous research (Vaessen et al., 2018). Briefly, fresh cultures were prepared from a frozen stock culture $\left(-80^{\circ} \mathrm{C}\right)$ on De Man Rogosa and Sharpe (MRS) (Merck, Germany) agar (Oxoid, United Kingdom) plates. Plates were incubated for $65-70 \mathrm{~h}$ at $30^{\circ} \mathrm{C}$ after which the plates were stored at $4{ }^{\circ} \mathrm{C}$ until further use for a maximum of three days. A single colony was used to inoculate $10 \mathrm{~mL}$ MRS broth and incubated statically for $24 \pm 2$ hours at $30^{\circ} \mathrm{C}$. After incubation, the culture was diluted 1:100 into MRS broth and incubated statically for $16-18$ hours at $30^{\circ} \mathrm{C}$. This stationary phase culture (9.5 log CFU/mL) was centrifuged for 10 minutes at $13,500 \times g$ at $20{ }^{\circ} \mathrm{C}$, and the resulting pellet was washed once with washing solution and subsequently suspended in electroporation medium, containing 0.3 M trehalose (Merck, Germany). The exact compositions of the washing solution and electroporation medium can be found in previous research (Vaessen et al., 2018).The final conductivity and $\mathrm{pH}$ of the suspended culture $(9.5$ $\log \mathrm{CFU} / \mathrm{mL}$ ) in electroporation medium at $21^{\circ} \mathrm{C}$ were $0.15 \mathrm{~S} / \mathrm{m}$ and $\mathrm{pH} 6.8$, respectively.

\subsubsection{Electroporation equipment and settings}

Electroporation was performed in disposable cuvettes with aluminium electrodes and an electrode distance of $2 \mathrm{~mm}$ using Gene Pulser Xcell equipment (Bio-Rad, USA), including the PC mode (Bio-Rad, USA). For all experiments, $400 \mu \mathrm{L}$ of culture at room temperature $( \pm$ $21^{\circ} \mathrm{C}$ ) was subjected to a square wave protocol with different voltages, pulse durations and number of pulses. The following voltages were used: 500, 1000, 1500, 2000 and $2500 \mathrm{~V}$ resulting in electric fields of $2.5,5.0,7.5,10.0$ and $12.5 \mathrm{kV} / \mathrm{cm}$. Pulse duration was set to 50 $\mu \mathrm{s}, 100 \mu \mathrm{s}$ or $1 \mathrm{~ms}$ and the number of pulses to $1,2,4$ or 10 . The pulse interval of the equipment between two pulses was $5 \mathrm{~s}$ by default. Multiple pulses were triggered by manual pressing the pulse button, leading to a pulse interval of 5-10 s. Resulting droop values (average decay of pulse height) were monitored and were at maximum $6 \%$ for pulses 
up to $100 \mu$ s duration and approximately $18 \%$ for pulses of 1 ms duration. These droop values were used to correct the electric field strengths that were used to calculate the specific energy input of the electroporation treatment according to equation 3.1. Here, $w$ represents the specific energy input $(\mathrm{J} / \mathrm{kg}), \sigma$ the culture conductivity $(\mathrm{S} / \mathrm{m}), E$ the electric field strength $(\mathrm{V} / \mathrm{m}), \tau$ the pulse duration (s), $n_{p}$ the number of pulses and $\rho$ density of the medium $\left(\mathrm{kg} / \mathrm{m}^{3}\right)$. For the density a value of $1039 \mathrm{~kg} / \mathrm{m}^{3}$ was used, which is the density of a $10(w / w) \%$ sucrose solution (Asadi, 2005). It is assumed similar to the density of our electroporation solution with approximately $10 \%$ trehalose.

$$
w=\frac{\sigma \cdot E^{2} \cdot \tau \cdot n_{p}}{\rho}
$$

Additionally, the maximum theoretical temperature increase was calculated on the basis of the energy input according to equation 3.2, in which $\Delta T$ is the temperature increase $(\mathrm{K})$ and $c_{p}$ the heat capacity of the liquid $(\mathrm{J} / \mathrm{kg} \cdot \mathrm{K})$. A heat capacity of $3950 \mathrm{~J} / \mathrm{kg} \cdot \mathrm{K}$ was used for the $10(\mathrm{w} / \mathrm{w}) \%$ trehalose medium, again similar to that for a $10(\mathrm{w} / \mathrm{w}) \%$ sucrose solution (Asadi, 2005).

$$
\Delta T=\frac{w}{c_{p}}
$$

\subsubsection{Fluorescence staining}

Analysis of membrane permeability was based on cell membrane permeability for the membrane impermeable stains propidium iodide (PI) and SYTOX Green (Invitrogen, USA). Several staining methods have been used for the different experiments. In the experiments with PI addition before and after PEF treatment, a counterstain is used to visualize all cells under the fluorescent microscope. This counterstain, SYTO 9 (Invitrogen, USA), is a nucleic acid stain able to penetrate intact membranes colouring all cells green. PI has a stronger nucleic acid binding affinity and can displace SYTO9 when entering the cell, therefore once cells take up $\mathrm{PI}$, the red fluorescence of $\mathrm{PI}$ is observed under the microscope (Stocks, 2004). A graphical overview of the two staining methods is shown in Fig. 3.1. The following sections will describe these methods in more detail. 


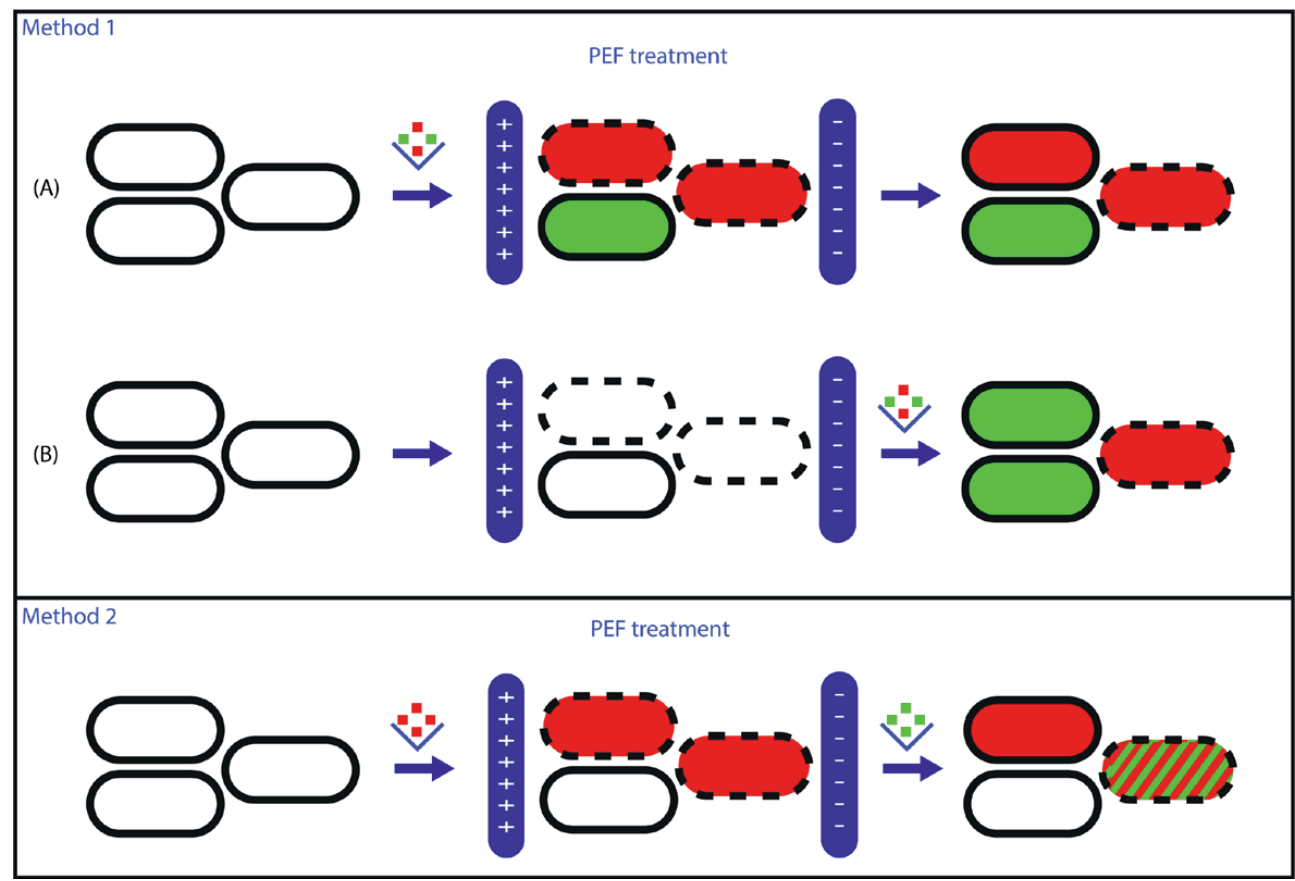

Figure 3.1 Schematic overview of the two staining methods used in this study. Dashed black lines represent damaged/permeabilized membranes and intact black lines represent intact cell membranes. In method 1 a mixture of PI (red) and counterstain SYTO 9 (green) was added either before PEF treatment ( $A$ ) or after PEF treatment (B). Reversibility of membrane integrity is based on the difference in PI uptake between $A$ and B. In method 2, the PI stain (red) was added before PEF and SYTOX Green (green) after the PEF treatment. Reversibility of membrane integrity is based on PI and/or SYTOX Green uptake. Coloured squares above the arrows indicate the moment in the procedure when the stain(s) were added to the culture.

\subsubsection{Addition of PI and counterstain SYTO 9 before or after electroporation}

The experiments with the addition of $\mathrm{PI}$ and counterstain SYTO 9 before or after electroporation were carried out with the use of a LIVE/DEAD staining kit (Invitrogen, USA). This kit contains the membrane permeable stain SYTO 9, which stains all cells green and the membrane impermeable stain propidium iodide (PI), which exhibits a bright red fluorescence signal upon binding to nucleic acids after entering the cell with permeabilized membrane. A culture suspended in electroporation medium was split into two samples, as indicated and visualised in figure 3.1-method 1. In the first sample (indicated as A) the stains were added before electroporation, and part of this sample was also kept as a control 
(without electroporation). In the second sample (indicated as B), the PI and SYTO 9 stains were added after electroporation. The final PI and SYTO 9 concentrations in both samples were $40 \mu \mathrm{M} \mathrm{PI}$ and 3.3 $\mu \mathrm{M}$ SYTO 9. Analysis of membrane permeability was performed with fluorescence microscopy after at least 10 minutes of incubation at room temperature in the same way as described previously (Vaessen et al., 2018). Briefly, 10-20 images with about 100-400 cells per image were captured per sample and subsequently the red and green cells on these images were counted using an image analysis routine as described by Perdana et al. (2012). The percentages of green cells on the images were averaged for each sample (control, electroporated with stain, stain added after electroporation) and subsequently these averages were used to determine the fractions of the culture that were already permeable before electroporation, irreversibly permeated, reversibly permeated or not permeated as described in Table 3.1.

Table 3.1 Determination of the unaffected, reversible, irreversible and initially permeabilized fractions of the cell population upon PEF treatment as analysed by the two different fluorescence staining methods.

\begin{tabular}{lll}
\hline & $\begin{array}{c}\text { Method 1 } \\
\text { PI before and after PEF with } \\
\text { counterstain SYTO 9 }\end{array}$ & $\begin{array}{c}\text { PI before and SYTOX } \\
\text { Green after PEF }\end{array}$ \\
\hline $\begin{array}{l}\text { Not permeabilized by PEF } \\
\text { (unaffected, intact } \\
\text { membrane) }\end{array}$ & $\begin{array}{l}\text { \% green cells in sample } \\
\text { stained before PEF }\end{array}$ & $\begin{array}{c}\text { \% cells low in PI and low in } \\
\text { SYTOX Green fluorescence }\end{array}$ \\
\hline $\begin{array}{l}\text { Reversible permeabilized } \\
\text { cells }\end{array}$ & $\begin{array}{l}\text { \% green cells in sample } \\
\text { stained after PEF minus } \\
\text { \% green cells in sample } \\
\text { stained before PEF }\end{array}$ & $\begin{array}{l}\text { \% cells high in PI, low in } \\
\text { SYTOX Green fluorescence }\end{array}$ \\
\hline $\begin{array}{l}\text { Irreversible } \\
\text { permeabilized cells }\end{array}$ & $\begin{array}{l}\text { \% green cells in control } \\
\text { sample minus \% green cells } \\
\text { in sample stained after PEF }\end{array}$ & $\begin{array}{l}\text { \% cells high in PI and high } \\
\text { in SYTOX Green } \\
\text { fluorescence }\end{array}$ \\
\hline $\begin{array}{l}\text { Cells already } \\
\text { permeabilized before PEF }\end{array}$ & $\begin{array}{l}\text { 100\% minus green cells in } \\
\text { control sample (no PEF) }\end{array}$ & n.a.* \\
$\begin{array}{ll}\text { *The fraction of cells already permeabilized before PEF was checked for every experiment in a control sample and } \\
\text { was < 2\% for all cultures }\end{array}$ &
\end{tabular}

\subsubsection{Addition of $\mathrm{PI}$ and SYTOX Green}

In the experiments with PI and SYTOX Green, both stains were added to the same electroporation cuvette at a different moment in the procedure (Fig. 3.1-method 2 for graphical overview). Before electroporation PI (Invitrogen, USA) was added to the culture 
in electroporation medium in a final concentration of $40 \mu \mathrm{M}$. Subsequently the sample was electroporated and kept at room temperature for approximately 10 minutes after electroporation, after which SYTOX Green (Invitrogen, USA) was added to the sample in a final concentration of $5 \mu \mathrm{M}$. Electroporated and control samples were subsequently analysed using a BD-FACS Aria III flow cytometer (instead of fluorescent microscopy) to be able to detect cells that took up both PI and SYTOX Green. First, the single cells were selected and determined using forward scatter (FSC) and side scatter (SSC) parameters. Second, 50,000 events per sample were analysed for their fluorescent properties using a 488-nm laser with a 502LP and 600 LP (selection range 502 to 600-nm) filter set for SYTOX Green and a 561-nm laser with a 600LP and 610/20 filter set for PI. The analysis of the different populations in the density plots was based on the PI and SYTOX Green fluorescence intensities as described in Table 3.1. To evaluate whether the use of flow cytometry instead of fluorescent microscopy imaging made a difference for the results obtained regarding membrane permeability, some experiments with PI addition before and after PEF treatment (method 1 ) were repeated and analysed with the flow cytometer and the flow cytometer gave similar results compared to the fluorescence microscopy analysis with respect to the percentages of unaffected, reversible and irreversible permeabilized cells.

\subsubsection{Survival analysis and selective plating}

In order to compare fluorescence staining results to culturability of the PEF treated cells, additional PEF experiments were performed and followed by plate counting. PEF treated and control samples were decimally diluted in phosphate buffered saline (PBS), plated on MRS agar plates and incubated under microaerobic conditions at $30^{\circ} \mathrm{C}$ for $2-4$ days (Vaessen et al., 2018). To determine the sublethally damaged fraction of cells that cannot grow out when an additional hurdle is present, MRS agar plates supplemented with $5(\mathrm{w} / \mathrm{v}) \% \mathrm{NaCl}$ were also prepared. Control and PEF treated cultures were plated on both MRS and MRS$\mathrm{NaCl}$ plates. These plates were incubated at $30{ }^{\circ} \mathrm{C}$ under microaerobic conditions for 3-5 days. After incubation, plates were counted and the counts of six replicate plates of each sample were averaged and used to determine the CFU/mL of the sample. Survival was calculated as a percentage of $\mathrm{CFU} / \mathrm{mL}$ of the PEF treated sample compared to the control sample. 


\subsubsection{Heat treatment}

Heat treatment experiments were performed to evaluate whether a temperature of $45^{\circ} \mathrm{C}$, which is the maximum theoretical temperature during PEF experiments, would result in $\mathrm{PI}$ uptake. Small volumes ( $50 \mu \mathrm{l}$ in a $1.5 \mathrm{ml}$ Eppendorf vial) of $L$. plantarum WCFS1 cultures in trehalose medium were incubated for 1 and 5 minutes in a water bath at $45{ }^{\circ} \mathrm{C}$ in the presence of $40 \mu \mathrm{M} \mathrm{PI}$ and $3.3 \mu \mathrm{M}$ SYTO 9.

\subsubsection{Experimental set-up}

All experiments were carried out at least in biologically independent duplicates, performed on different days with another pre-culture. For each independent experiment, the measured result was again obtained by analysis of multiple cells or samples (for example, 2,000-5,000 cells for fluorescence microscopy, 50,000 cells for flow cytometry and six replicate plates for plate counting). Average values of the independent replicates are presented with error bars indicating the standard deviation of these biologically independent replicates. Significance was tested with a Student's $t$-test, using a P value of 0.05 .

\subsection{Results and discussion}

\subsubsection{Assessment of reversibility of membrane permeabilization}

Membrane permeability of $L$. plantarum WCFS1 was evaluated for various electric field strengths by evaluating PI uptake before and after PEF treatment. Four different fractions could be distinguished; cells with an unaffected intact membrane after PEF, cells with a reversible permeabilized membrane for $\mathrm{PI}$, cells with an irreversible permeabilized membrane and cells that were already permeable for PI before PEF (staining method 1 in Fig. 3.1). L. plantarum WCFS1 became permeable for PI after PEF treatment using an electric field strength of $7.5 \mathrm{kV} / \mathrm{cm}$ or higher when 2 pulses of $100 \mu$ s were applied (Fig. 3.2A). At $7.5 \mathrm{kV} / \mathrm{cm}$ approximately $10 \%$ of the population was reversible permeabilized and a similar percentage was irreversible permeabilized for PI. At higher electric field strengths, the irreversible permeabilized fraction of the population increased, while the reversible permeabilized fraction of the population remained approximately $10 \%$. This increase in irreversibly permeabilized cells at higher electric field strengths is in line with the survival results based on plate counts (Fig. 3.2B). Also, it is in line with previous research on inactivation of microorganisms using PEF treatment, where a higher electric field strength 
led to an increased microbial inactivation (Abram et al., 2003; Cebrián et al., 2016; Jaeger et al., 2009).

A

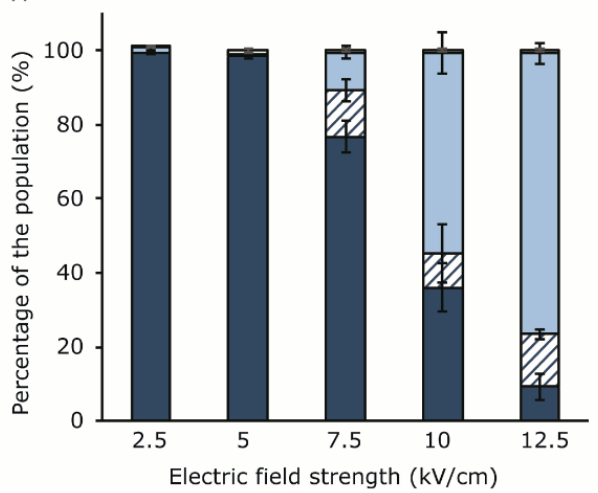

- Unaffected $/ /$ Reversible

Irreversible $\square$ Initially permeabilized

C

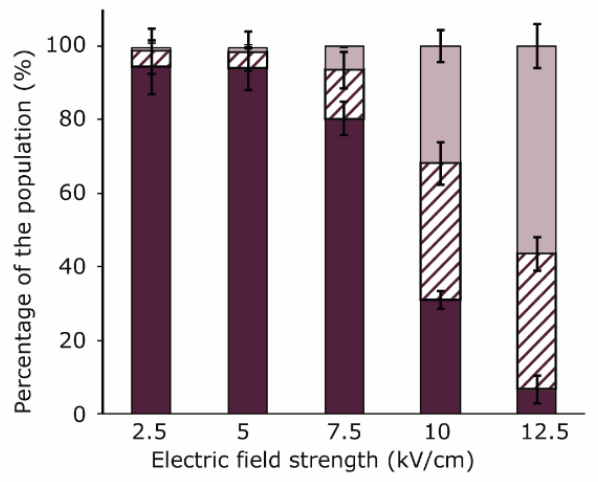

Unaffected $\%$ Reversible

Irreversible
B

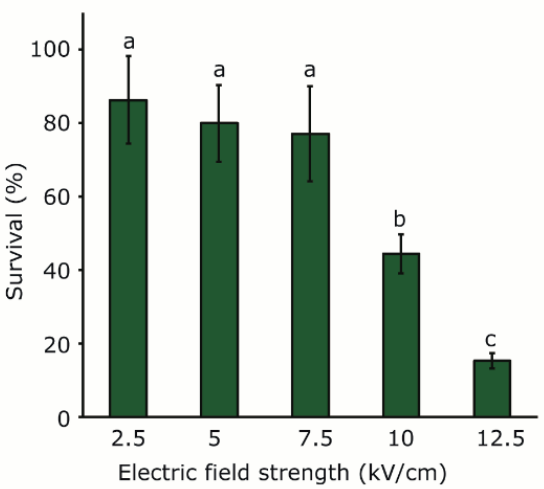

D

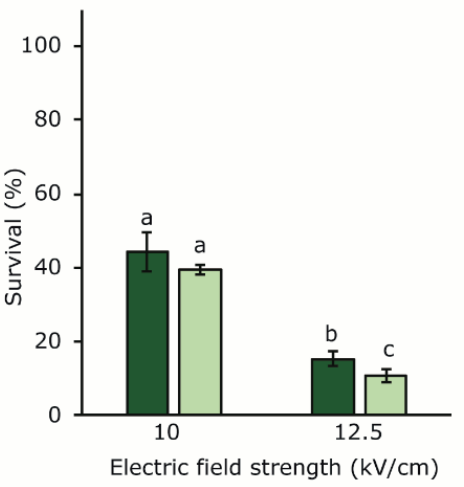

- MRS MRS- $\mathrm{NaCl}$

Figure 3.2 Membrane permeabilization and survival after PEF treatments at various electric field strengths. A: membrane permeabilization assessed by PI addition before or after PEF treatment, B: Survival after PEF treatment assessed by plate counting (adapted from Vaessen et al. (2018)) C: Membrane permeabilization assessed by combination staining with PI and SYTOX Green, D: Survival assessed by plating on MRS and MRS-NaCl plates. For all PEF treatment two square wave pulses of $100 \mu$ s duration were applied. Error bars represent standard deviations of biologically independent samples $(n \geq 2)$ and different letters indicate significant differences $(P<0.05)$. 
In order to apply reversible PEF treatment for loading of viable bacterial cells with small molecules, it is important that the reversible permeabilized cells survive the PEF treatment. However, we cannot draw a conclusion on the survival of the relatively small fraction of reversible permeabilized cells in Fig. 3.2A by comparing these results with survival results based on plate counts in Fig. 3.2B. The evaluation of plate counts on this percentage scale is limited due to the sensitivity of the plating method.

The membrane permeability results of the second staining method, with the addition of PI before PEF and SYTOX Green after PEF treatment (Fig. 3.2C), resulted in very limited membrane permeability for electric field strengths up to $7.5 \mathrm{kV} / \mathrm{cm}$, and was only slightly higher compared to the first method (Fig. 3.2A). Interestingly, at higher electric field strengths a larger fraction of reversible permeabilized cells was found with the double staining method. In this method, the different fractions were obtained using flow cytometry as can be seen in more detail in Fig. 3.3. Fig. 3.2C shows that instead of a reversible permeabilized fraction of approximately $10 \%$ quantified using method 1 , method 2 showed that this part of the population increased to approximately $40 \%$ at $10 \mathrm{kV} / \mathrm{cm}$. This raises the question which of the two methods gives a better representation of the reversibility of membrane permeabilization upon PEF treatment.

As an additional control, also staining with SYTOX Green before and after PEF has been performed for PEF treatments at 7.5 and $10 \mathrm{kV} / \mathrm{cm}$. These experiments at $10 \mathrm{kV} / \mathrm{cm}$ resulted in a reversible permeabilized fraction which was slightly larger than with the addition of PI before and after PEF, but smaller compared to the combination staining method. At 7.5 $\mathrm{kV} / \mathrm{cm}$ no clear differences between the methods were observed (Supplementary material S.3.1). It could be reasoned that using a combination staining method is less suitable, because the two stains could compete for the same binding sites on the DNA. However, Müller et al. (2010) described that no displacement between SYTOX Green and PI was observed in their experiments when these stains were used together, indicating that these stains probably have different binding sites in the DNA. Our own additional experiment with a mixture of live and dead cells confirmed this finding (Supplementary material S.3.2). Also, the decreased effectiveness of nucleic acid stains when DNA is damaged might play a role in the observed effect (Lebaron et al., 1998), as PEF could damage DNA (Delimaris et al., 2006). 
Another method which is often used to detect sublethal damage of bacteria during PEF treatment is selectivity plating (Ulmer et al., 2002). Although this method is based on outgrowth rather than membrane permeability, it might give an indication of what is more likely; a large or small reversible permeabilized part of the population? Therefore, control and PEF treated samples at 10 and $12.5 \mathrm{kV} / \mathrm{cm}$ were plated on both MRS and MRS- $\mathrm{NaCl}$ plates. For both PEF conditions only small differences, which were significant only at 12.5 $\mathrm{kV} / \mathrm{cm}$, were observed in survival between MRS and MRS-NaCl plates (Fig. 3.2D). These small differences are more in line with membrane permeability based on only PI staining (method 1) than with the combination staining with PI and SYTOX Green (method 2). Based on a comparison of the double staining method (Fig. 3.2C) with the survival results (Fig. $3.2 B, D)$, it can be concluded that not all the cells that were reversible permeabilized according to this double staining method were able to form colonies.

\subsubsection{Membrane permeabilization and population properties}

A big advantage of the membrane permeability assessment with the double staining method with PI and SYTOX Green is the possibility for more in depth analysis on the characteristics of the three different subpopulations. The three subpopulations; namely intact cells, reversible permeabilized cells and irreversible permeabilized cells, are categorised based on the PI and SYTOX Green fluorescence intensities, as shown in the density plots in Fig. 3.3. As an extra check, the subpopulations have also been determined based on the separate PI and SYTOX Green signals, which resulted in similar percentages of the different fractions for all conditions (data not shown). The membrane permeability results that were derived from these density plots are shown in Fig. 3.2C. 

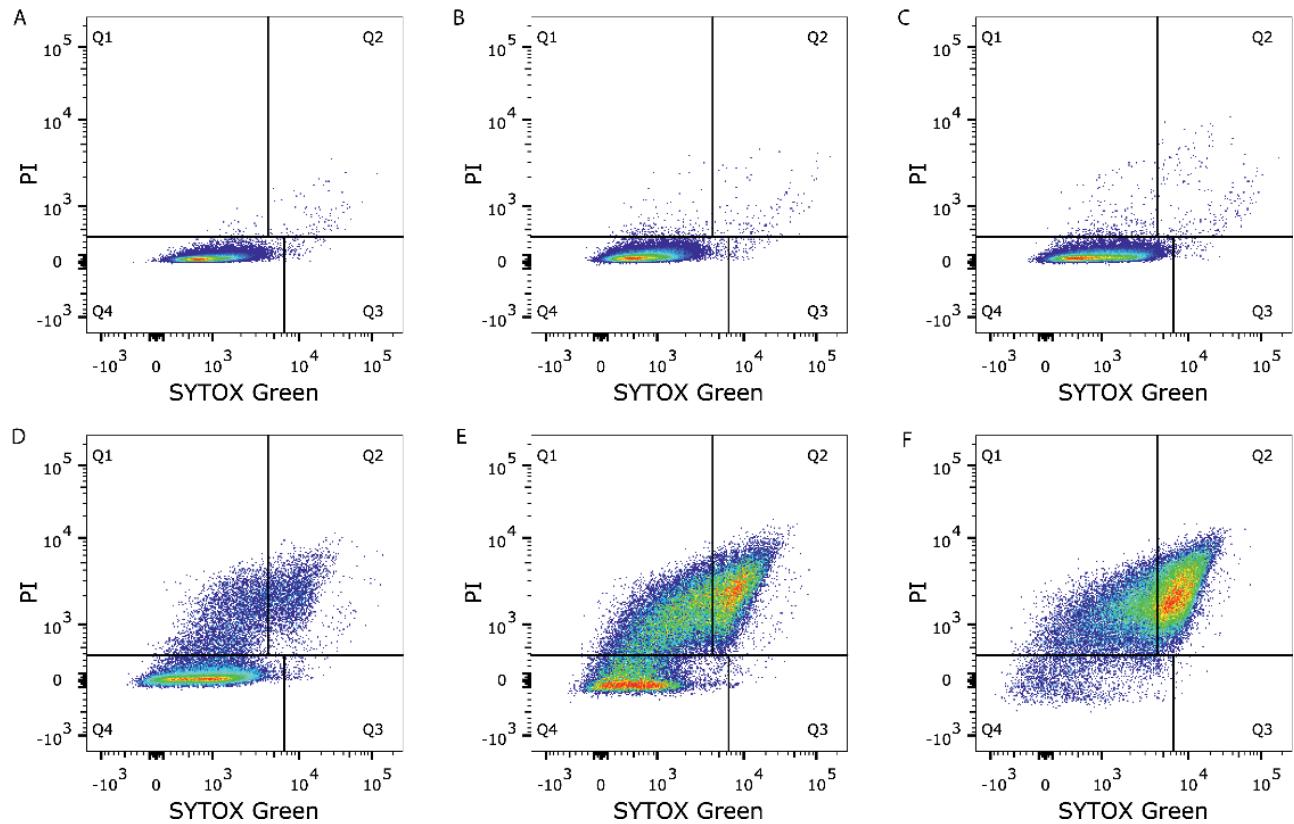

Figure 3.3 Fluorescence density plots obtained by flow cytometry of the bacterial population stained with PI and SYTOX Green without electroporation $(A)$ and electroporated with two pulses of $100 \mu$ s at 2.5 (B), 5 (C), 7.5 (D), 10 (E) and 12.5 (F) kV/cm. Each dot represents a single bacterial cell. The colours represent a density scale from purple/blue (low) to red (high cell density). The quadrants represent the different fractions of the population: Q1: reversible permeabilized, Q2: irreversible permeabilized and Q4: unaffected intact membrane. These density plots are presented as an example from one of the experiments of which the results are presented in Fig. 3.2C

As can be seen in figure 3.3, the increase in PI and/or SYTOX Green fluorescence intensity is not at the same level for every cell, but covers a range of signal intensities. With increasing the electric field strength, the population shifted towards higher PI and SYTOX Green intensities, indicating a shift from unaffected cells (low PI, low SYTOX Green) to reversible permeabilization (high PI, low SYTOX Green), and to irreversible permeabilization (high PI, high SYTOX Green).

Subsequently, the forward scatter (FSC) values, which are determined from the light scatter of a bacterial cell measured in the path of the laser, were assessed for the three different subpopulations. The FSC value can give an indication of the bacterial cell size, where lower FSC values correspond to smaller sized cells (Bouvier et al., 2001; Koch et al., 1996). However, this relation between FSC and cell size does not always hold, since cell 
morphology might also affect FSC values. Interestingly, the mean and median FSC values differed between the three subpopulations. The intact cells that did not take up any stain after PEF had lower mean FSC values than the reversible permeabilized cells, which again had lower FSC values than the irreversible permeabilized cells (Fig. 3.4). This trend was observed at 7.5, 10 and $12.5 \mathrm{kV} / \mathrm{cm}$, though these differences in FSC value were not in all cases significant. The data for the median FSC showed the same trends (data not shown). The relative mean FSC was calculated by dividing the specific FSC value of the subpopulation of interest by the FSC value of the entire population (unaffected + reversible + irreversible). As a control, the mean and median FSC values of the whole electroporated cell population were compared to those of a cell population without electroporation. The mean and median FSC values for both populations were similar, thus the electroporation treatment itself did not affect the FSC values. The FSC results showed larger mean and median FSC-values for the irreversible fraction, suggesting that the larger cells in the population are relatively more affected by the PEF treatment compared to the smaller cells. Wouters et al. (2001b) observed a similar relation between FSC and PI uptake after electroporation in Lactobacillus plantarum LA10-11, though they did not take the reversible fraction into account, as PI was only added after electroporation. Interestingly, the mean FSC value of the reversible permeabilized fraction was for all PEF conditions between the values for the irreversible and unaffected cell fractions. The underlying mechanism for this observation remains to be determined, though this observation triggered us to speculate about it. These results suggest that reversibility of permeabilization is due to a balance between the intensity of the PEF treatment and the bacterial cell size of the individual cells. It is probably not related to a specific fraction of the cells that more easily recovers their membrane integrity. In other words; the cells that were reversibly permeabilized after PEF treatment at $7.5 \mathrm{kV} / \mathrm{cm}$ were very likely to be irreversibly permeabilized at higher electric field strengths, and cells that were unaffected at $7.5 \mathrm{kV} / \mathrm{cm}$ might be reversible permeabilized at higher electric field strengths. 


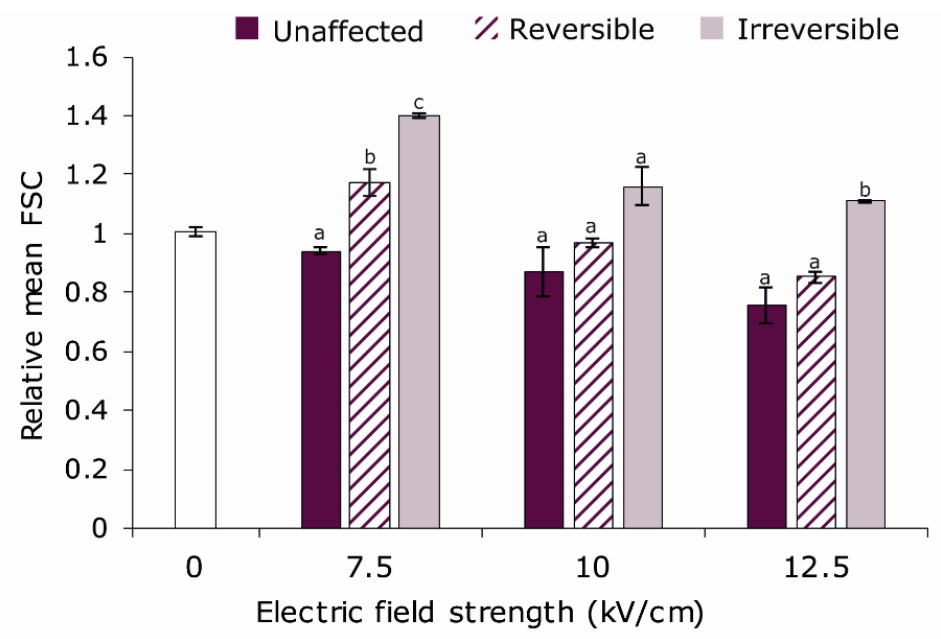

Figure 3.4 Relative mean forward scatter (FSC) signals for three different fractions of the population; unaffected intact cells, reversible permeabilized cells and irreversible permeabilized cells. The different groups are determined based on PI and SYTOX Green staining and relative mean FSC values are determined by dividing the mean FSC signals of specific populations by the mean FSC value of the entire cell population. The data point presented at $0 \mathrm{kV} / \mathrm{cm}$ is a control; namely the mean FSC of the control culture divided over the mean FSC of the PEF treated cultures. Error bars represent standard deviations of biologically independent duplicates and different letters indicate significant differences $(P<0.05)$ between the three fractions at one specific electric field strength.

The FSC results in this study are in line with previous research on the relation between the efficacy of a PEF treatment and cell size, where in general it was observed that the larger the cell the more easy it is permeabilized upon a specific PEF treatment (Agarwal et al., 2007; Heinz et al., 2001). However, it should be noted that this relation between cell size and efficacy of PEF does not always hold when comparing different bacterial strains to each other (García et al., 2005). Other factors such as pre-culture conditions, Gram-positive or Gram-negative or cell morphology can play a role as well.

\subsubsection{Effect of pulse parameters on reversibility of membrane permeabilization}

In addition to electric field strength, other pulse parameters could influence reversibility of membrane permeabilization as well. Therefore, we studied the effect of the number of pulses and pulse duration on reversibility of membrane permeabilization. One, two, four and ten pulses of $100 \mu \mathrm{s}$ at $7.5 \mathrm{kV} / \mathrm{cm}$ were applied (Fig. 3.5A), as well as one pulse of $1 \mathrm{~ms}$ at 5 and $7.5 \mathrm{kV} / \mathrm{cm}$ (Fig. 3.5B). Similar to the results presented in Fig. 3.2, the reversible 
permeabilized fraction remained small (maximum $10 \%$ of the population) for all these PEF conditions when the membrane permeability was assessed with the addition of PI before and after PEF treatment (Fig. 3.5A, B), and increase of the intensity of the treatment led only to an increase in the irreversible fraction. Other studies did find a larger reversible permeabilized fraction of the population reaching up to $80 \%$ in microalgae and mammalian cells (Bodénès et al., 2019; Silve et al., 2016). Also for another Gram-positive bacterium, Listeria monocytogenes, reversible permeabilized fractions for PI reached up to $40 \%$ of the population (García et al., 2007). However, it is noted that comparison with similar studies is not that obvious, since small differences in experimental set-up may lead to completely different outcomes, i.e. microbial and culture characteristics, PEF treatment medium, specific PEF conditions.

A

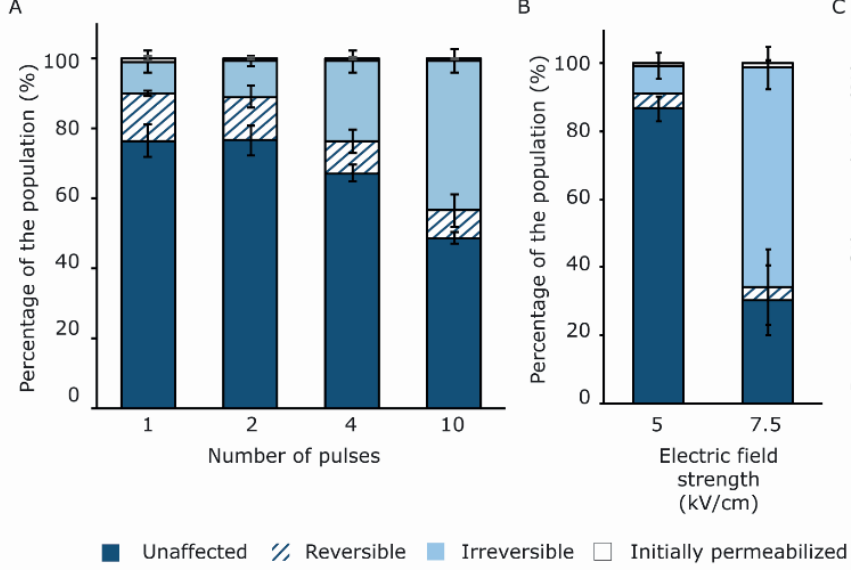

C

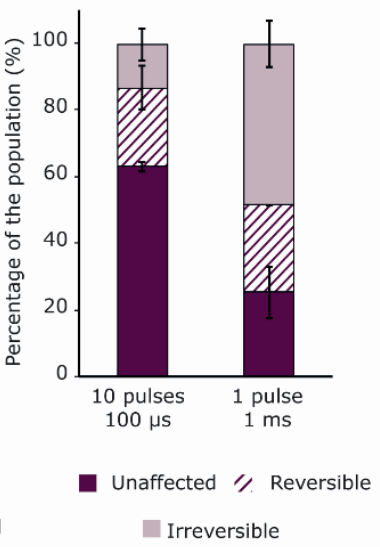

Figure 3.5 Membrane permeabilization upon PEF treatment assessed by PI staining before and after PEF (A, B) and by double staining with PI and SYTOX Green (C). A: PEF treatments at $7.5 \mathrm{kV} / \mathrm{cm}$ with $100 \mu$ s pulse duration and varying number of pulses. B: PEF treatment at two electric field strengths with one pulse of $1 \mathrm{~ms}$ duration. C: PEF treatments at $7.5 \mathrm{kV} / \mathrm{cm}$. Error bars represent standard deviations of biologically independent replicates $(n \geq 2)$.

PEF treatments at $7.5 \mathrm{kV} / \mathrm{cm}$ with 10 pulses of $100 \mu \mathrm{s}$ (Fig. 3.5A) and with one pulse of $1 \mathrm{~ms}$ (Fig. 3.5B) were compared having the same total pulse duration and similar energy input, but different number of pulses. Interestingly, one pulse of $1 \mathrm{~ms}$ resulted in more irreversible permeabilization compared to 10 pulses of $100 \mu \mathrm{s}$. One longer pulse is apparently more effective for irreversible membrane permeabilization than 10 shorter pulses at the same 
electric field strength, though the reversible permeabilized fractions for both conditions remained comparable at approximately 4 - 8\% (Fig. 3.5A, B). This effect of more irreversible permeabilization using pulses of $1 \mathrm{~ms}$ compared to shorter pulses of 15 or $100 \mu \mathrm{s}$ at the same energy input is in line with recent observations for inactivation of bacteria and yeast in fruit juices (Timmermans et al., 2019). When comparing the same PEF conditions with the double staining method (Fig. 3.5C), a larger reversible permeabilized fraction was observed for this second method, similar to our earlier observations presented in Fig. 3.2. However, the difference between 10 pulses of $100 \mu$ s and 1 pulse of $1 \mathrm{~ms}$ remained similar with this method, showing that one pulse of $1 \mathrm{~ms}$ was more effective in terms of irreversible membrane permeabilization.

The different settings of the PEF parameters, namely electric field strength, pulse duration and number of pulses applied in this study led to different energy inputs. The energy input for each PEF condition was calculated according to equation 3.1 and compared to the percentages of the population that remained unaffected (Fig. 3.6). Increasing energy input did not necessarily decrease the percentage of the population with an intact membrane. Also, the electric field strength played a role, as at the same specific energy input a higher electric field strength led to a lower number of cells with an intact cell membrane (Fig. 3.6A). These results confirm what has been found in studies on inactivation of bacteria and yeasts, where a higher electric field strength results in more microbial inactivation at a similar energy input (Heinz et al., 1999; Pataro et al., 2011). Whereas a clear relation was observed between electric field strength and membrane permeability, this was not the case for pulse duration and membrane permeability (Fig. 3.6B). Here, a large variation in the percentage of the population with intact membrane was found for example for pulses of $100 \mu \mathrm{s}$ with similar energy input. This variation shows that pulse duration is not the main PEF parameter affecting membrane permeability and confirms that changing the electric field strength has more influence. 
A

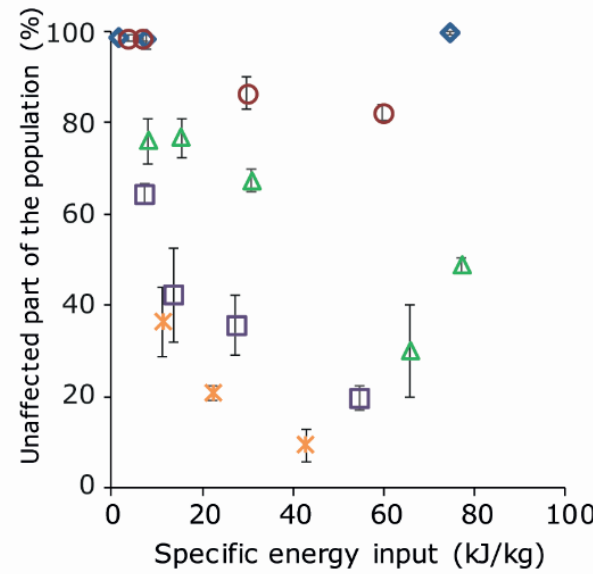

B

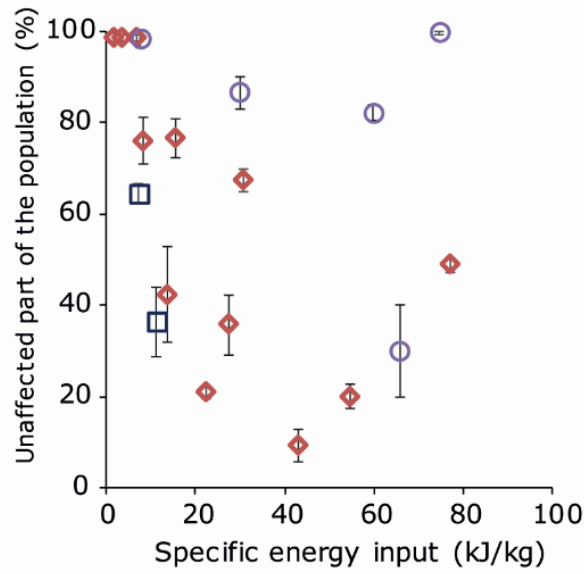

Figure 3.6 Percentage of the L. plantarum WCFS1 culture with an unaffected intact cell membrane after PEF treatment as assessed by PI staining for several PEF conditions. Both panels show the same experimental data presented in different ways. In figure $A$ the symbols represent the different electric field strengths $(\diamond 2.5 \mathrm{kV} / \mathrm{cm}, 05 \mathrm{kV} / \mathrm{cm}, \Delta 7.5 \mathrm{kV} / \mathrm{cm}, \square 10$ $\mathrm{kV} / \mathrm{cm}, \times 12.5 \mathrm{kV} / \mathrm{cm}$ ) and in figure $B$ the different symbols represent the pulse duration of a single pulse ( $\square 50 \mu \mathrm{s}, \diamond 100 \mu \mathrm{s}$, $01 \mathrm{~ms})$. Error bars represent standard deviations of biologically independent replicates $(n \geq 2)$.

Energy input can also be related to temperature increase (equation 3.2). Temperature increase during electroporation could potentially also contribute to cell inactivation and/or membrane permeabilization. The theoretical temperature increase at $100 \mathrm{~kJ} / \mathrm{kg}$ energy input would be $25^{\circ} \mathrm{C}$, resulting in a theoretical temperature of approximately $45^{\circ} \mathrm{C}$. To assess whether the temperature increase alone would result in PI uptake, cultures of $L$. plantarum WCFS1 stained with PI were incubated in a water bath at $45^{\circ} \mathrm{C}$ for 1 and 5 minutes. Also after 5 minutes still more than $98 \%$ of the cell population remained impermeable for PI (data not shown) and therefore the temperature increase alone is not considered to influence the membrane permeability results. However, an additive effect of the temperature increase to the PEF treatment, especially at higher energy inputs, cannot be entirely excluded based on the synergistic effect often described in PEF studies for inactivation purposes (Aronsson and Rönner, 2001; Timmermans et al., 2014).

Based on the membrane permeabilization results at different field strengths presented in Fig. 3.6A, there seems to be a critical electric field strength around $5 \mathrm{kV} / \mathrm{cm}$ that is required 
to permeabilize the membrane of Lactobacillus plantarum WCFS1. Based on previous research, a certain transmembrane potential difference is required for pore formation in the cellular membrane (Escoffre et al., 2009; Tsong, 1991). The transmembrane potential difference that corresponds to the observed critical field strength can be estimated from these field strengths in combination with the cell size and shape according to equation 3.3. In this equation, $\Delta \varphi$ is the transmembrane potential difference $(\mathrm{V}), f(A)$ a dimensionless shape factor for non-spherical cells, $A_{F}$ the distance from the center of the cell to the cell membrane in the direction of the electric field $(\mathrm{m})$ and $E$ the electric field strength $(\mathrm{V} / \mathrm{m})$ (Toepfl et al., 2007; Zimmermann et al., 1974).

$$
\Delta \varphi=-f(A) \cdot A_{F} \cdot E
$$

The shape factor can be approximated from the cell dimensions as described in equation 3.4 , in which $l$ is the length of the bacterial cell $(\mathrm{m})$ and $d$ the diameter $(\mathrm{m})$ (Hülsheger et al., 1983).

$$
f(A)=l /(l-0.33 \cdot d)
$$

Based on the cell size estimations for L. plantarum WCFS1 from microscopy pictures (being $1.9 \mu \mathrm{m} \times 0.8 \mu \mathrm{m}$ ) and when assuming the cell orientation to be in parallel with the direction of the electric field, a critical electric field strength of $5 \mathrm{kV} / \mathrm{cm}$ corresponds to a transmembrane potential difference of $552 \mathrm{mV}$. This transmembrane potential difference required for pore formation in the cell membrane has been determined before for several cell types and was found to be $240 \mathrm{mV}$ for Chinese hamster ovary cells (Teissié and Rols, 1993), 150-500 mV for bilayer lipid vesicles (Tsong, 1991) and between 260-1300 mV for different bacteria depending on the pre-culture conditions and bacterial strain (Hülsheger et al., 1983). Additionally, a more recent microfluidic study found a critical electric field strength for SYTOX Green uptake around $5 \mathrm{kV} / \mathrm{cm}$ for the Gram positive bacterium Corynebacterium glutamicum (Garcia et al., 2016). Overall, the determination of the critical electric field strength and the transmembrane potential calculated from this field strength remain a rough approximation, since it depends on several assumptions, i.e. the cell orientation in the electric field and the method of evaluating PEF efficacy (e.g. fluorescent stains or plate counts). These assumptions, the cell types and pre-culture conditions differ between the studies and therefore should be considered when conclusions about a critical 
electric field strength or transmembrane potential difference to induce pore formation are made.

\subsection{Conclusion}

Reversibility of membrane permeabilization upon PEF treatment was assessed with two different staining methods. One method was based on the use of a single stain, employing two parallel PEF treatments and the other one was based on a double staining approach using one PEF treatment. These two methods resulted in similar trends regarding the effect of the different PEF parameters on membrane permeabilization. Remarkably, clear differences between these two methods were found in the quantified fraction considered as reversible permeabilized. These differences indicate that the choice of fluorescent marker(s) can influence the conclusions drawn on the effect of a PEF treatment, e.g. the effect of PEF on enhancing molecule transport into the cells, advocating the use of different staining methods in parallel. Still, fluorescent staining techniques remain important for understanding the effect of specific PEF treatments on cells. The double staining method allowed us to also compare the forward scatter values of the different subpopulations, which provided an indication of differences in cell size and effect of permeabilization. Regarding the different PEF conditions evaluated in this study a critical electric field strength for membrane permeabilization is observed around $5 \mathrm{kV} / \mathrm{cm}$ for L. plantarum WCSF1. In addition, increase of the electric field strength above this critical value resulted in more membrane permeabilization when compared to other PEF conditions with a similar energy input.

\section{Acknowledgements}

This work was carried out within the framework of the Institute for Sustainable Process Technology (ISPT), The Netherlands. Partners in this project are Danone, DSM, Cosun, NIZO Food Research, Winclove and Wageningen University and Research. The authors would like to thank Letao Han and Niels van Mossevelde for their help with the experimental work. 


\section{Supplementary information}

\section{S.3.1 Third staining method: SYTOX Green before and after PEF}

As an additional control, some experiments have been performed with the addition of SYTOX Green before and after PEF treatment. The principle of this method is the same as the method with the addition of PI before and after PEF (described in the materials and methods section). The results presented in Fig. S.3.1 were obtained from two biologically independent samples, for both replicates all three the staining methods have been performed and analysed by flow cytometry.

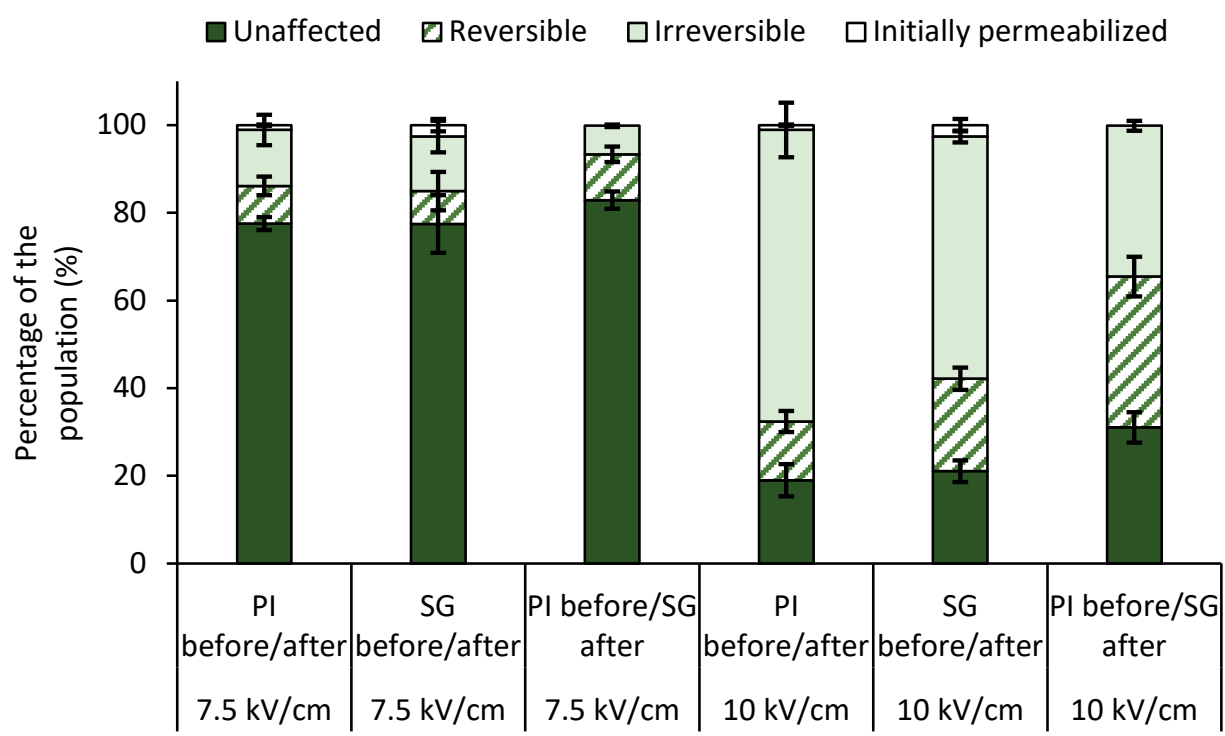

Figure S.3.1 Membrane permeabilization of L. plantarum WCFS1 after PEF treatment at 7.5 and $10 \mathrm{kV} / \mathrm{cm}$ assessed by three different staining methods, all analysed by flow cytometry.

As can be seen in Fig. S.3.1, the membrane permeability results at $7.5 \mathrm{kV} / \mathrm{cm}$ did not significantly differ between the three staining methods. However, at $10 \mathrm{kV} / \mathrm{cm}$ the addition of PI before and SYTOX green after PEF resulted in the largest reversible permeabilized fraction compared to the other two staining methods. Staining with SYTOX Green before and after PEF resulted in a slightly larger reversible permeabilized fraction compared to 
staining with PI before and after PEF, though not as large as with the double staining method.

\section{S.3.2 PI and SYTOX Green displacement?}

An initial staining experiment with a mixture of live and dead cells was performed to check the effect of the combination staining method (dead cells were heat treated at $70^{\circ} \mathrm{C}$ for 8 minutes). In these experiments we found that all dead cells clearly increased in both $\mathrm{PI}$ and SYTOX green signals as can be seen in Fig. S.3.2, indicating that no (complete) displacement between these two stains took place.

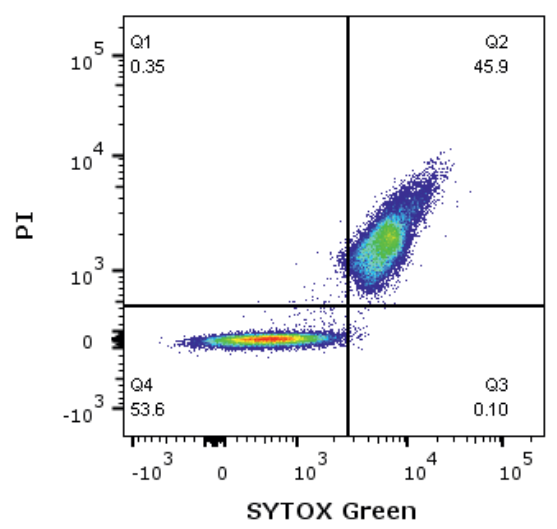

Figure S.3.2 Fluorescence density plot obtained by flow cytometry of a mixture of live and heat-killed L. plantarum WCFS1 cells stained with PI and SYTOX Green. Each dot represents a single bacterial cell. The colours represent a density scale from blue (low) to red (high cell density). 
. 


\section{4}

\section{Accumulation of intracellular trehalose and lactose in Lactobacillus plantarum WCFS1 during pulsed electric field treatment and subsequent freeze and spray drying}

This chapter has been published as Vaessen, E.M.J., den Besten, H. M. W., Esveld, E. D. C., \& Schutyser, M. A. I. (2019). Accumulation of intracellular trehalose and lactose in Lactobacillus plantarum WCFS1 during pulsed electric field treatment and subsequent freeze and spray drying. LWT, 115, 108478 


\begin{abstract}
Survival of bacteria during drying processes is required for products such as probiotics or starter cultures. In earlier studies high intracellular trehalose concentrations have been related to processing robustness during freezing and drying for several cell types. In this study we evaluated pulsed electric field (PEF) pre-treatment to increase intracellular trehalose in Lactobacillus plantarum WCFS1 and subsequent survival during freeze drying and storage in a trehalose matrix. Surprisingly, despite increased intracellular trehalose by PEF pre-treatment no enhanced survival was observed after drying and storage. Additional analysis of intracellular trehalose before and after freeze drying and spray drying revealed that during the drying process intracellular trehalose concentrations increased. Due to this increase, the intracellular trehalose concentrations in the PEF pre-treated and control samples did not differ anymore. To explain what happens during the drying processes, results for trehalose were compared to similar experiments with lactose. A remarkable difference was observed between transfer of trehalose and lactose into cells during the freezing step before freeze drying, while both disaccharides gave similar cellular protection when used as freezing and drying matrix. Generated knowledge on transport of disaccharides into bacterial cells during freezing and drying can benefit processing of living bacterial formulations.
\end{abstract}




\subsection{Introduction}

Freeze drying and spray drying are commonly used drying methods for long term preservation of probiotics and starter cultures. Survival of the bacteria during drying and subsequent storage is often required for their function as fermentation inoculum or in the gastrointestinal tract. Therefore a lot of studies focus on improving survival of probiotics upon drying and storage of the powders (Huang et al., 2017; Peighambardoust et al., 2011; Schutyser et al., 2019). A common approach to increase survival during drying is to embed bacteria in a protective carrier matrix. The carrier matrix consists often of carbohydrates such as maltodextrins, trehalose and lactose (Broeckx et al., 2017; Huang et al., 2017). Mechanisms that can explain the protective effect of these carbohydrates are the water replacement and glass transition theories. The water replacement theory states that carbohydrates take the place of the water molecules and by that stabilize the structure of the phospholipid cell membrane during drying. The glass transition theory states that the bacteria are embedded in a vitrified matrix, which protects the bacteria.

Several scientific studies showed that the intracellular presence of high amounts of carbohydrates can also contribute to increased survival of bacteria during drying (Leslie et al., 1995; Termont et al., 2006). Also for yeast, mammalian and plant cells high intracellular trehalose concentrations have been observed to enhance robustness during freezing or drying stress (Chen et al., 2001; Phoon et al., 2008; Tapia et al., 2015) . Moreover, several organisms, among which several bacteria, are able to accumulate trehalose intracellularly under desiccation stress, enabling them to survive in harsh environments (Zhang and Yan, 2012). Presence of carbohydrates on both sides of the membrane could not only protect the bacterial membrane, but also cytosolic proteins (Mensink et al., 2017). However, actual measurements of intracellular carbohydrate concentrations after the drying treatment are lacking. Though, there are some studies that investigated the effect of increased intracellular trehalose concentrations on drying or cryoprotection of bacteria. These studies measured intracellular trehalose concentrations only before the drying or freezing process (Duong et al., 2006; Termont et al., 2006). Duong et al. (2006) found that trehalose uptake and hydrolysis genes play a critical role in survival of Lactobacillus acidophilus NCFM after freezing and thawing. Survival after freezing and thawing decreased with one log in mutants with insertions to impair the function of these genes compared to the wild type. To study the effect of intracellular trehalose on freeze drying survival, Termont et al. (2006) 
introduced trehalose synthesis genes in L. lactis. The subsequent accumulation of intracellular trehalose resulted in increased survival after freeze drying in a skim milk matrix. Intracellular trehalose concentrations can also be increased by a mechanical method, i.e. pulsed electric field (PEF) treatment (Vaessen et al., 2018). During PEF treatment trehalose diffuses into the cells through pores in the membrane that are formed by exposure of the cells to an electric field. When the membrane is not damaged too much, the pores can reseal and the cell contains an increased intracellular trehalose concentration.

In this study we investigated the hypothesis that intracellular trehalose delivered to the cells by PEF treatment enhances survival after drying and storage. Lactobacillus plantarum WCFS1 was chosen as model probiotic in this study, since this strain is widely studied on its drying behaviour and exposure to PEF (Kleerebezem et al., 2003; Perdana et al., 2014b, 2013; Vaessen et al., 2018). Freeze drying of Lactobacillus plantarum WCFS1 with and without PEF pre-treatment was followed by four months of storage. Subsequently, the intracellular trehalose concentrations were measured before and after each process step in order to understand the effect of intracellular trehalose during drying. In the end these intracellular trehalose concentrations were compared to a second disaccharide, lactose, which was also used as protective agent and a second drying process, spray drying, to get a more mechanistic understanding of intracellular carbohydrate concentrations during drying processes.

\subsection{Materials and methods}

\subsubsection{Bacteria and pre-culture conditions}

Lactobacillus plantarum WCFS1 was pre-cultured as described in our previous study (Vaessen et al., 2018). Briefly, a liquid culture was prepared by inoculating a single colony into $10 \mathrm{~mL}$ MRS broth (Merck, Germany). After incubation at $30^{\circ} \mathrm{C}$ for $24 \pm 2$ hours, this culture was diluted 1:100 in MRS broth for the experiments followed by overnight (16-18 hours) static incubation at $30^{\circ} \mathrm{C}$ before starting the experiments.

The overnight culture was centrifuged (Thermo-Fischer Scientific, USA) at 13,500 $\times g$ for 10 minutes at $20^{\circ} \mathrm{C}$. The $\mathrm{pH}$ of the supernatant was measured (Toledo Inlab Expert, Switzerland) and was $3.9 \pm 0.1$ for all experiments. The resulting pellet was washed once with washing solution and after centrifugation suspended in the drying medium containing either trehalose or lactose. This resulted in a culture in drying medium containing $3-4 \cdot 10^{9}$ 
$\mathrm{CFU} / \mathrm{mL}$. The washing solution consisted of $5 \mathrm{mmol} / \mathrm{L} \mathrm{NaCl}, 5 \mathrm{mmol} / \mathrm{L} \mathrm{Na} 2 \mathrm{HPO}_{4} \cdot 2 \mathrm{H}_{2} \mathrm{O}, 5$ $\mathrm{mmol} / \mathrm{L} \mathrm{NaH} \mathrm{PO}_{4} \cdot \mathrm{H}_{2} \mathrm{O}$ and $0.5 \mathrm{mmol} / \mathrm{L} \mathrm{MgCl} 2 \cdot 6 \mathrm{H}_{2} \mathrm{O}$ in demineralized water. The drying media were prepared by dissolving either $0.3 \mathrm{~mol} / \mathrm{L}$ trehalose (Merck, Germany) or 0.3 $\mathrm{mol} / \mathrm{L}$ lactose monohydrate (VWR, USA) in washing solution. The resulting conductivity of the drying medium with the bacteria was $0.15 \mathrm{~S} / \mathrm{m}$.

\subsubsection{PEF treatment}

Bacteria in the drying medium were PEF treated in a batch PEF system (IXL, The Netherlands) with a custom made treatment chamber (Fig. 4.1). The treatment chamber consists of two vertical parallel titanium electrodes, sealed by a silicone $3.0 \mathrm{~mm}$ spacer (Fig. $4.1 \mathrm{~B}, \mathrm{C})$. The remaining space between the electrodes was filled with approximately $13 \mathrm{~mL}$ of culture. The cultures were PEF treated with 10 square wave pulses of $6.3 \mathrm{kV} / \mathrm{cm}$ and a pulse duration of $100 \mu$ s with a pause time of 5 seconds in between the pulses. The pulse shape was monitored via an oscilloscope attached to the system (Fig. 4.1A,D). All PEF treatments were performed at a constant temperature of $21^{\circ} \mathrm{C}$. This temperature was controlled with a thermostatted water bath attached to the aluminium heating block of the treatment chamber. After PEF treatment, part of the culture was used for survival assessment and intracellular trehalose measurements and the other part was used in further freeze drying experiments.

\subsubsection{Freezing and freeze drying}

Bacterial cultures in the drying medium with and without PEF pre-treatment were frozen at $-20^{\circ} \mathrm{C}$ prior to freeze dying. All freezing and freeze drying experiments were performed with $1 \mathrm{~mL}$ culture in a $30 \mathrm{~mL}$ container, leading to a thin layer of liquid on the bottom of the jar and a big surface area. Samples with and without PEF pre-treatment of the same biological replicate were freeze dried in parallel in a Christ Epsilon 2-6D freeze dryer (Martin Christ, Germany). After freeze drying the resulting powders had a moisture content of maximum $6 \mathrm{w} / \mathrm{w} \%$ as analysed by a moisture analyser (Sartorius, Germany). Survival and intracellular trehalose analysis were performed as described in section 2.6 and 2.7. 

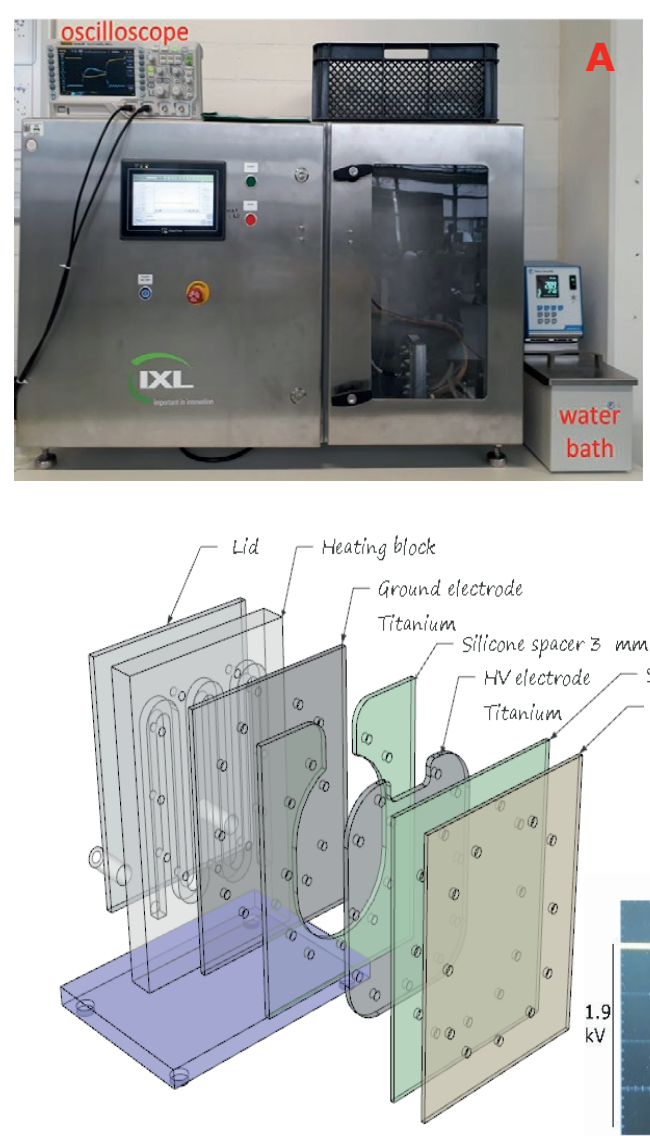

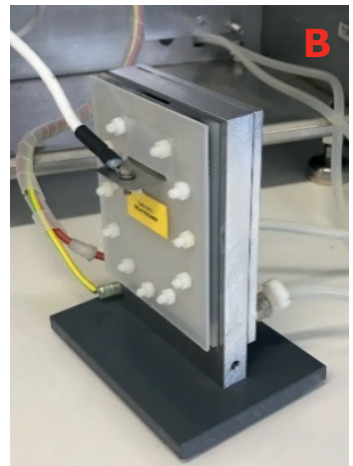

C

Figure 4.1 IXL batch PEF system, A: Overview of PEF set-up including pulse generator, treatment chamber, water bath and oscilloscope, B: CUstom-made PEF treatment chamber assembled, C: Exploded view of the parallel plate PEF treatment chamber, D: typical pulse applied in this study.

\subsubsection{Storage tests}

Bacterial cultures with and without PEF pre-treatment in the trehalose drying medium were frozen at $-20^{\circ} \mathrm{C}$ and freeze dried as described before. The freeze dried cultures were stored in desiccators at room temperature $\left(21^{\circ} \mathrm{C}\right)$. One desiccator contained an oversaturated lithium chloride solution $\left(a_{w}=0.11\right)$ and the other desiccator contained an oversaturated potassium acetate solution $\left(a_{w}=0.23\right)$. Both desiccators were closed with a vacuum of 100 mbar. Samples for survival analysis were taken approximately every 25 days for four months. 


\subsubsection{Spray drying}

Bacterial cultures in the drying medium were spray dried in a Büchi B-290 laboratory spray dryer (Büchi Labortechnik AG, Switzerland). The inlet temperature was set to $120^{\circ} \mathrm{C}$ and the flow rate to $15 \%$, which led to an outlet temperature of $67-70^{\circ} \mathrm{C}$. For each experiment, 50-60 mL of culture was spray dried. The resulting powder was collected and had a moisture content of maximum $6 \mathrm{w} / \mathrm{w} \%$. For further analysis the spray dried powder was distributed in samples of $100 \pm 3 \mathrm{mg}$, which were subsequently reconstituted and analysed as further explained in section 2.6 and 2.7 .

\subsubsection{Survival analysis}

Bacterial cultures were decimally diluted in peptone physical salt solution (PPS) (Tritium Microbiologie B.V., The Netherlands) and subsequently plated on MRS agar. Both diluting and plating were done in duplicate. Plates were incubated in micro-aerobic jars for 48-96 hours at $30^{\circ} \mathrm{C}$. Samples were taken before and after each process step (e.g. PEF treatment, freezing, drying). The freeze dried and spray dried samples were reconstituted in $10 \mathrm{~mL}$ PPS, resulting in the $10^{-1}$ dilution before further dilution and plating. Survival is presented as percentage of surviving $\mathrm{CFU} / \mathrm{mL}$ after the treatment (PEF, freezing, drying) compared to the initial CFU/mL. For the storage tests, the $\log _{10} \mathrm{CFU} / \mathrm{mL}$ of the different samples at every time point is presented.

\subsubsection{Intracellular trehalose and lactose measurements}

Intracellular trehalose and/or lactose extraction of culture samples was performed in the same way as described in Vaessen et al. (2018). Briefly, extracellular sugars were washed away with three washing steps in PBS. Washing efficiency was regularly tested by measuring the disaccharide concentrations in the supernatant of the third washing step. After the third washing step, the cells were suspended in milliQ water and intracellular components were extracted by disrupting the cells in a bead beater. Cell extract samples were frozen until HPLC analysis. Disaccharide extraction and survival analysis were performed in parallel on the same treated cultures.

Dried samples were reconstituted in $1 \mathrm{~mL}$ PBS before immediately continuing with the trehalose/lactose extraction as described. Next to the samples reconstituted in PBS, some samples were reconstituted in the trehalose drying medium or lactose drying medium to evaluate the reconstitution effect as indicated in the specific sections. 
HPLC analysis was performed with a ICS5000 High Performance Anion Exchange Chromatography system in combination with a Pulsed Amperometric Detector (HPAECPAD) (Dionex Corporation, USA). The flow rate was $0.3 \mathrm{~mL} / \mathrm{min}$ and the column temperature $20^{\circ} \mathrm{C}$. The mobile phases were $0.1 \mathrm{~mol} / \mathrm{L} \mathrm{NaOH} \mathrm{(A)} \mathrm{and} \mathrm{a} \mathrm{gradient} \mathrm{of} 1 \mathrm{~mol} / \mathrm{L} \mathrm{NaAc}$ in 0.1 $\mathrm{mol} / \mathrm{L} \mathrm{NaOH}$ (B). Chromatograms of samples were compared to calibration curves of trehalose and lactose to determine the disaccharide concentrations with the use of Chromeleon software.

\subsubsection{Propidium iodide uptake during freezing}

Propidium iodide (Invitrogen, USA) to a final concentration of $40 \mu \mathrm{M}$ was added either before freezing of a culture in trehalose medium, after freezing and in a control sample that was kept at room temperature during the experiment. One millilitre samples were frozen at $-20^{\circ} \mathrm{C}$ and after approximately 1 hour samples were thawed and analysed for PI uptake. The fraction of cells that took up PI and exhibited a higher red fluorescence signal was measured with a BD FACS Aria III flow cytometer.

\subsubsection{Experimental set-up}

Freeze drying and spray drying experiments were performed at least in duplicate with each duplicate being a biologically independent sample cultured and dried on another day. Survival during storage tests was assessed by duplicate measurements at each time point. Significance was tested with a Student's $t$-test, using a P-value of 0.05 .

\subsection{Results and discussion}

\subsubsection{Effect of pulsed electric field pre-treatment on viability of $L$. plantarum WCFS1 during drying and storage}

Lactobacillus plantarum WCFS1 cultures in a trehalose matrix with and without PEF pretreatment were freeze dried and the obtained bacterial powders were stored at two different water activities. The survival of both PEF pre-treated and control cultures was determined to relate the effect of intracellular trehalose concentrations to survival after drying and storage. (Fig. 4.2). PEF pre-treated cultures had an increased intracellular trehalose concentration that was approximately eight-fold higher compared to the control samples without PEF pre-treatment, similar to the results presented in the first two columns in figure 4.3A. Survival directly after PEF pre-treatment was around $80 \%$ (Fig.S.4.1). It is 
noted that the increased intracellular trehalose concentration after PEF pre-treatment is most probably present in only part of the viable bacterial population as discussed in previous research (Vaessen et al., 2018). This part of the population with much higher intracellular trehalose concentrations is hypothesized to survive better during drying and subsequent storage. Because trehalose is not present in the whole population and the bacteria survive the drying process relatively well, we expected that the effect of this increased intracellular trehalose is especially visible after a substantial decrease in survival, i.e. in the order of $\log (\mathrm{s})$. Approximately $70 \%$ of the $L$. plantarum WCFS1 cells survived the freeze drying treatment (Fig. 4.3B) with, as expected, no significant difference between the PEF pre-treated sample and the control.

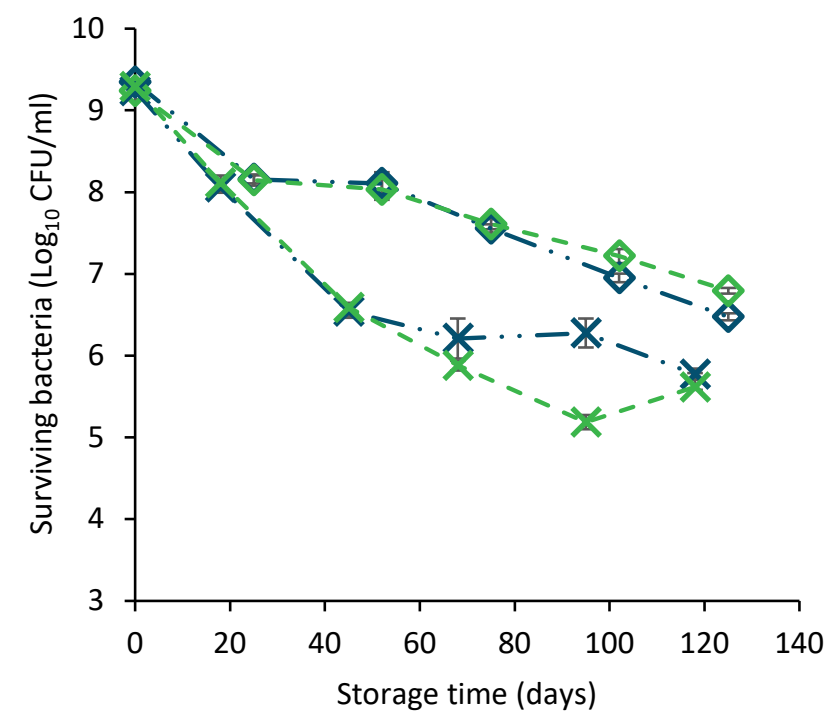

Figure 4.2 Number of surviving freeze-dried L. plantarum WCFS1 cells during storage at different water activities with and without PEF pre-treatment. Control $(\diamond)$ and PEF pretreated $(\diamond)$ culture stored at $a_{w}=0.11$ and control $(\mathbf{X})$ and PEF pre-treated $(\mathbf{X})$ culture stored at $a_{w}=0.23$. Cultures were freeze dried in a trehalose matrix before storage. Error bars indicate standard deviations of duplicate measurements.

During four months of storage at room temperature the number of bacteria in samples with initially $9.3 \log \mathrm{CFU} / \mathrm{mL}$ decreased to 5.6 or $6.7 \log \mathrm{CFU} / \mathrm{mL}$ for storage at water activities of 0.23 and 0.11 , respectively (Fig. 4.2). Better survival of bacteria stored at lower water 
activities is in line with previous research (Abe et al., 2009; Higl et al., 2007). Interestingly, survival during storage of the freeze-dried cultures was similar for the PEF pre-treated cultures and the control cultures without PEF pre-treatment (Fig. 4.2). These results are not in line with the initial hypothesis, and suggest that increased intracellular trehalose concentrations in PEF pre-treated cultures do not lead to a higher survival during freeze drying and subsequent storage in this case. Therefore, further research was performed on the intracellular trehalose concentrations during the different steps of the freeze drying process. In addition to trehalose, lactose was used for this study, because lactose was not detected intracellularly in Lactobacillus plantarum WCFS1 after growth and it is a relevant disaccharide that is often used as drying matrix for protection of bacteria during drying.

\subsubsection{Intracellular trehalose and lactose during freeze drying}

Intracellular trehalose concentrations were measured during the different steps of the freeze drying process (Fig. 4.3A). The analysis of the intracellular sugar required reconstitution of the dried powder to enable extraction of the intracellular sugar. The presented data for the dried powder are thus the intracellular concentrations after reconstitution in the same liquid volume. The effect of this reconstitution step on the results is further discussed in section 4.3.4. Please note that all intracellular concentrations in this study are presented as disaccharide concentrations in the cell extracts and not per cell. This is done because it is unknown whether these disaccharides are present in all cells or in a specific fraction of the cell population. Remarkably, the intracellular trehalose concentration in the control sample increased greatly after the initial freezing step before the freeze drying process. This increase resulted in a similar concentration of intracellular trehalose for the samples with and without PEF pre-treatment. The intracellular trehalose concentrations of the PEF pre-treated and control cultures remained similar also after subsequent freeze drying. This observation can explain the absence of any enhanced effect of the PEF pre-treatment on the survival during the storage tests. 

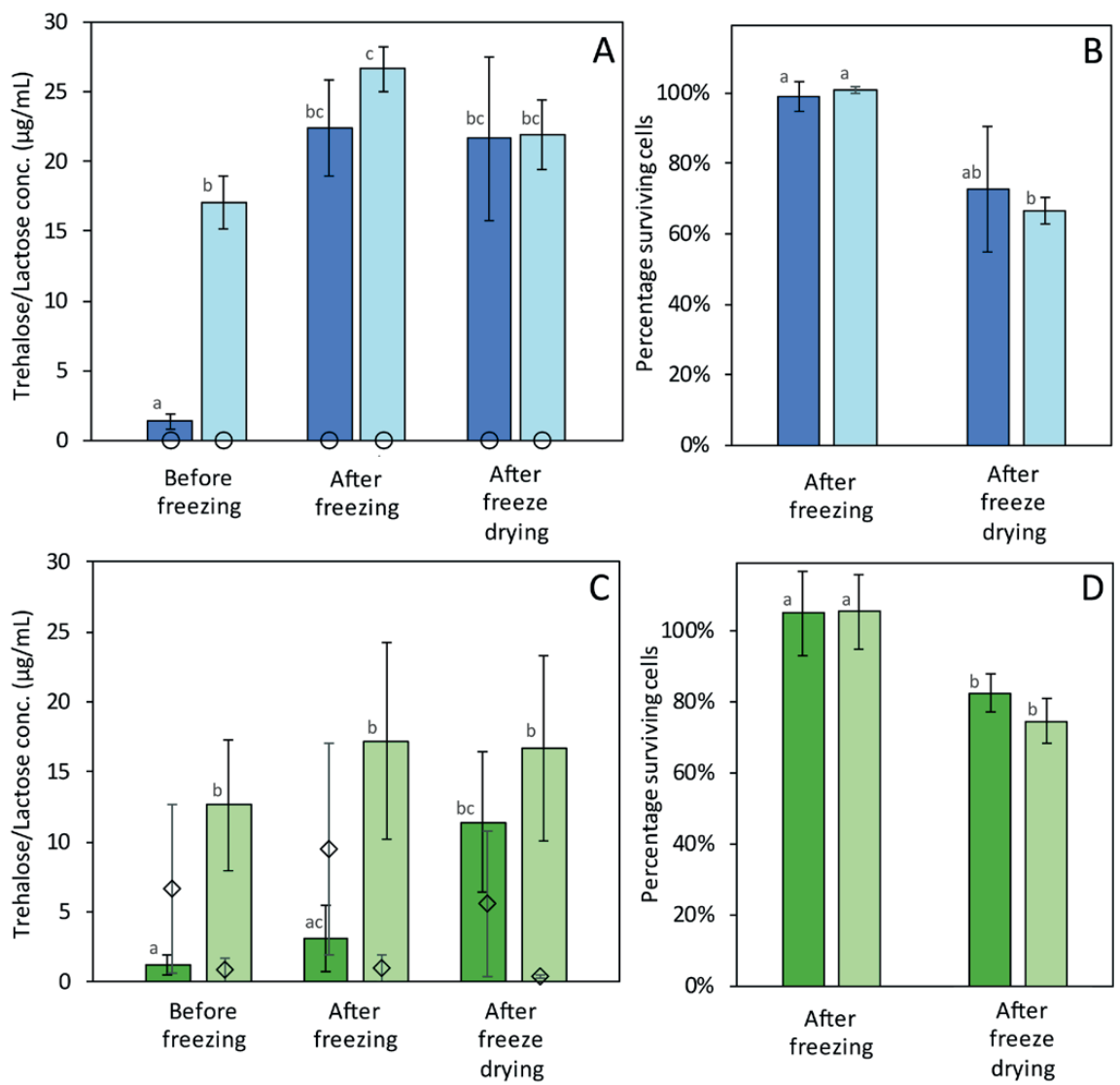

Figure 4.3 Intracellular disaccharide concentrations and survival of L. plantarum WCFS1 after freezing and freeze drying in trehalose medium ( $A$ and $B$ ) and lactose medium ( $C$ and D) with and without PEF pre-treatment. A: Trehalose concentration in control (blue bars) and PEF pre-treated (light-blue bars) samples. Symbols (o) show lactose concentrations in the same samples. C: Lactose concentrations measured in control (green bars) and PEF pretreated (light-green bars) samples. Symbols $(\diamond)$ show trehalose concentrations in the same samples. $B$ \& D: Corresponding survival after freezing and freeze drying in respectively trehalose and lactose medium. Intracellular concentrations are shown in $\mu \mathrm{g} / \mathrm{mL}$ sample, each sample contained $\sim 3 \cdot 10^{9} \mathrm{CFU} / \mathrm{mL}$. Error bars indicate standard deviations of independent samples, $n \geq 2$. Superscripts indicate significant differences between the bars.

The same experiments were repeated with another disaccharide, namely lactose. PEF pretreatment increased the intracellular lactose concentration in the cells, similar to the results in trehalose. However, when performing the freeze drying experiment in lactose (Fig. 4.3C), we did not observe a significant increase in intracellular lactose after the initial freezing 
step. Only after freeze drying the intracellular lactose increased compared to the initial concentration before freezing in the sample without PEF pre-treatment. This intracellular lactose concentration was slightly lower than the intracellular lactose concentration in the PEF pre-treated cells after freeze drying, though these concentrations did not differ significantly. This increase in intracellular lactose after drying suggested that the sugar may enter the cell during the drying process. During the drying process the concentration of the sugar on the outside of the cell increases greatly upon the removal of water. The drying process could have affected the cell membrane structure due to the removal of water resulting in a higher membrane permeability (Leslie et al., 1995). The effect of drying on the cell membrane is further discussed in section 4.3.3.

The difference in intracellular trehalose and lactose concentrations after the freezing step is remarkable. Normally one would not expect these disaccharides, having both a molecular weight of $342 \mathrm{Da}$, to migrate through a cell membrane. However, upon freezing the cell membrane goes through a gel-fluid transition or is exposed to ice crystals, which may make the cell membrane leaky upon which components can diffuse down their concentration gradient into the cell (Leslie et al., 1995). However, if this is the case, the concentration increase would be expected for both trehalose and lactose. Additional experiments with propidium iodide (PI, $668 \mathrm{Da}$ ) as a fluorescent marker molecule showed no PI uptake at all upon freezing of the cultures (Fig. S.4.2). There was no increase in red fluorescence signal of the frozen sample compared to the control (non-frozen) sample using the flow cytometer. Of course, it must be taken into account that PI is approximately twice as large in molecular weight compared to a disaccharide. Concluding, this result together with the difference between trehalose and lactose uptake after freezing at least suggests that complementary factors than a permeable cell membrane may be held responsible for trehalose uptake.

Complementary to passive transport via a permeable membrane, also a biological transport process could induce trehalose uptake of the cells during freezing. The genome of $L$. plantarum WCFS1 has been annotated and contains a wide variety of sugar uptake genes (Kleerebezem et al., 2003), among which a PTS trehalose transport system and a symporter of the GPH family which can also transport trehalose into the cell. The trehalose transport into the cells might also be affected by freezing. Duong et al. (2006) showed that transport of trehalose into the cell and subsequent hydrolysis is important for freeze-thaw survival of 
Lactobacillus acidophilus NCFM. Mutants with knockouts in these trehalose uptake and hydrolysis genes showed approximately one log decrease in survival, whereas $80-90 \%$ of the wild type survived several freezing and thawing cycles in a trehalose matrix.

The involvement of cellular transport is also suggested from measurements of trehalose concentrations in the samples that were PEF pre-treated and dried in the presence of lactose. In these samples, especially in the non-PEF treated, non-frozen samples, substantial amounts of trehalose were found intracellularly (Fig. 4.3C). These cultures were grown for 16 hours in MRS broth after which a relatively high intracellular trehalose concentration was found in these cells (data not shown). However, every time when suspending these cells in a trehalose medium, which was also done for the non-PEF treated cells as control sample, the intracellular trehalose concentrations were observed very low (Fig. 4.3A). A biological mechanism which might explain this difference in intracellular trehalose is called catabolite repression. Catabolite repression refers to the repression of the metabolism of other carbohydrates when the preferred carbohydrate (e.g. glucose) is present in the medium. In this case, it would imply that trehalose hydrolysis is repressed when glucose, which is the main sugar in MRS, or another preferred sugar is present in the medium. Previous research found that the proteome profile of the carbohydrate metabolism in $L$. plantarum indeed differs when using different media, indicating that catabolite repression is found in L. plantarum strains (Siragusa et al., 2014). Because of these remarkable findings on intracellular trehalose, the comparison to a second disaccharide, namely lactose, was investigated. Lactose was not present intracellularly after growth in MRS broth and, as expected, therefore also not present in cells which were dried in the presence of trehalose. It requires further study to understand which exact biological mechanisms could play a role in the intracellular accumulation of sugars during freezing.

\subsubsection{Intracellular trehalose and lactose during spray drying}

Compared to freeze drying, spray drying is a very fast drying process. Given the fast drying rate during spray drying, biological mechanisms (active transport) facilitating uptake of sugars during spray drying would not be expected to play a major role (Jahreis et al., 2008; Perdana et al., 2013). Therefore, we studied the intracellular disaccharide concentrations after spray drying and compared these results to the results after freeze drying. After spray drying in trehalose we observed a large increase in intracellular trehalose in L. plantarum WCFS1 (Fig. 4.4A). Spray drying in lactose resulted also in a similar large increase in 
intracellular lactose. The survival after spray drying in lactose and trehalose was similar for both disaccharides, $70-80 \%$ of the cells survived this drying treatment.
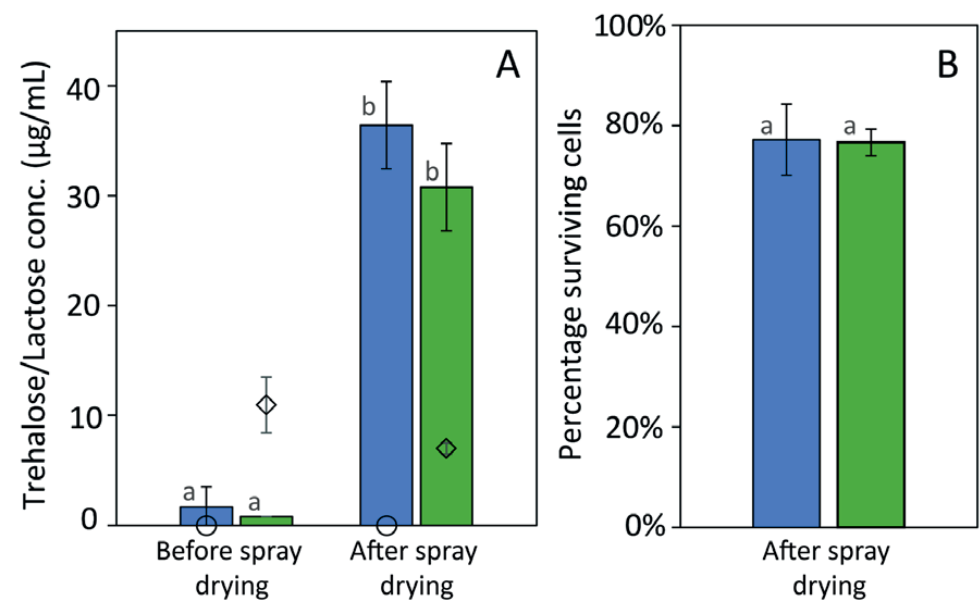

Figure 4.4 Intracellular disaccharide concentration before and after spray drying (A) and survival after spray drying (B) of cultures dried in trehalose (blue) or lactose (green). Symbols in figure $A$ show the lactose concentration in the trehalose sample (o) and the trehalose concentration in the lactose sample $(\diamond)$. Intracellular concentrations are shown in $\mu \mathrm{g} / \mathrm{mL}$ sample, each sample contained $\sim 3 \cdot 10^{9} \mathrm{CFU} / \mathrm{mL}$. Error bars represent standard deviations of biologically independent duplicates and superscripts indicate significant differences between the bars.

The total intracellular trehalose and lactose concentrations after spray drying were higher than after freeze drying, while the survival of the bacteria after both drying processes, irrespective of the disaccharide used in the drying matrix, did not differ significantly. The difference in intracellular trehalose and lactose between both drying processes might indicate that the type of drying process affects the intracellular accumulation of the disaccharides. Comparatively, in the laboratory-scale spray dryer that was used for these experiments the drying time is in the order of 1 - 1.5 seconds (Perdana et al., 2013), whereas the drying time of the freeze drying process was 45 hours. During dehydration of the cells a gel-fluid phase transition in the lipid bilayer can contribute to increased permeability of the cell membrane and may eventually lead to inactivation of the cells (Bryant et al., 2001; Crowe et al., 1989; Leslie et al., 1995). For spray drying, we found considerable accumulation of intracellular disaccharides, which indeed indicates that the phospholipid 
membrane was permeable to disaccharides during the drying treatment and/or subsequent reconstitution. For freeze drying in lactose the same phenomenon, although to a lesser extent, was observed, whereas in trehalose the increase was already observed during freezing, as discussed in section 3.2.

\subsubsection{Effect of drying or measurement method?}

The measured intracellular disaccharide concentrations in cells after drying could potentially be a side effect of the measurement procedure to determine intracellular trehalose and lactose. To measure intracellular disaccharide concentrations, extracellular disaccharides were washed away. The washing efficiency was evaluated by measuring the disaccharide content in the supernatant of the third washing step. Hypothetically, we could have measured sugars adsorbed to the cell that could not be washed away, instead of intracellular sugars. However, based on the sugar permeability of the peptidoglycan layer surrounding the cell membrane (Demchick and Koch, 1996), together with the low concentrations before treatment and the commonly known permeabilization effect of PEF treatment, the measured disaccharide concentrations are likely to be present in the cytoplasm of the bacteria.

Another essential step for the intracellular disaccharide extraction before HPLC analysis is reconstitution of the dried bacteria. The reconstitution step itself could also lead to increased intracellular concentrations because the drying matrix (e.g. lactose or trehalose) was still present in the powder. Furthermore, just like during the drying treatment, during reconstitution the cell membrane can undergo a phase transition (Crowe et al., 1989). In general, all samples were reconstituted in PBS before measuring the intracellular trehalose and lactose contents. However, to evaluate the effect of the reconstitution step we also reconstituted several samples in trehalose and/or lactose solutions instead of PBS (Table 4.1). These results indicate that the reconstitution could also lead to an increase in intracellular disaccharide concentration. For example, after spray drying in trehalose and reconstituting the sample in a lactose solution, both intracellular trehalose and lactose concentrations increased compared to the samples before spray drying (Table 4.1). Though, the increase of the disaccharide that was present in the drying matrix was clearly higher than the increase of the reconstitution disaccharide. This difference is already an indication that both during drying and during reconstitution disaccharides can enter the cell. To further evaluate the effect of the reconstitution step we dried cells without a disaccharide 
present in the drying matrix. When freeze drying the cells without a sugar present in the drying matrix and reconstituting them in either PBS, trehalose or lactose solutions, the trehalose or lactose concentrations in the samples reconstituted in that specific disaccharide slightly increased compared to reconstitution in PBS (Table 4.1). However, the intracellular disaccharide concentrations in the dried samples that were reconstituted in a disaccharide solution were rather low compared to the concentrations found when a sample was dried in the presence of a disaccharide (Table 4.1 and Fig. 4.3). These results indicate that the reconstitution step contributes to the measured increase in intracellular disaccharides after drying presented in figure 4.3 and 4.4 , although to a lesser extent compared to the drying itself.

\subsection{Conclusion}

PEF pre-treatment applied to increase intracellular trehalose concentrations did not enhance robustness of L. plantarum WCFS1 during drying and subsequent storage. Remarkably, intracellular trehalose and lactose also increased after freezing and/or drying without PEF pre-treatment. This increase could explain why PEF pre-treatment did not show an enhanced robustness. Knowledge on intracellular accumulation of sugars could be used to develop drying processes aiming at increased intracellular sugars and enhanced survival of bacteria after drying. 
Table 4.1 Summary of the reconstitution experiments performed to evaluate the effect of the reconstitution step on intracellular trehalose ( $\left.C_{\text {trehalose}}\right)$ and lactose (Clactose) concentrations. Concentrations are disaccharide concentrations measured in the sample with each sample containing $\sim 3 \cdot 10^{9} \mathrm{CFU} / \mathrm{mL}$ before drying. Detection limit of the method is $0.3 \mu \mathrm{g} / \mathrm{mL},<0.3$ indicates that the sample did not contain detectable amounts of the disaccharide.

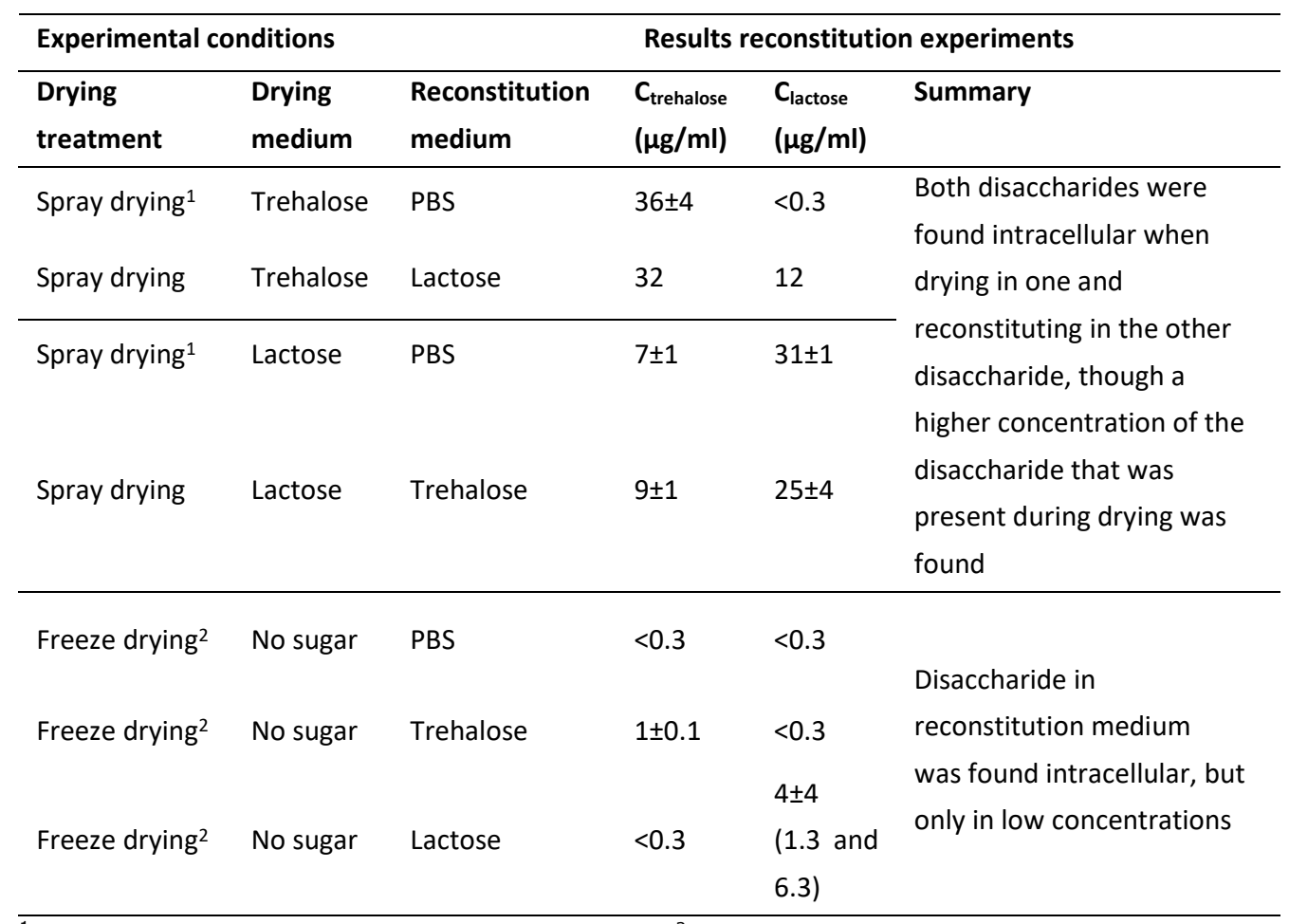

${ }^{1}$ The data in these rows are also presented in Fig. 4.4A. ${ }^{2}$ Survival after freeze drying without any sugar in the drying medium was $42 \pm 11 \%$ 


\section{Supplementary information}

\section{S4.1. Survival after PEF treatment}

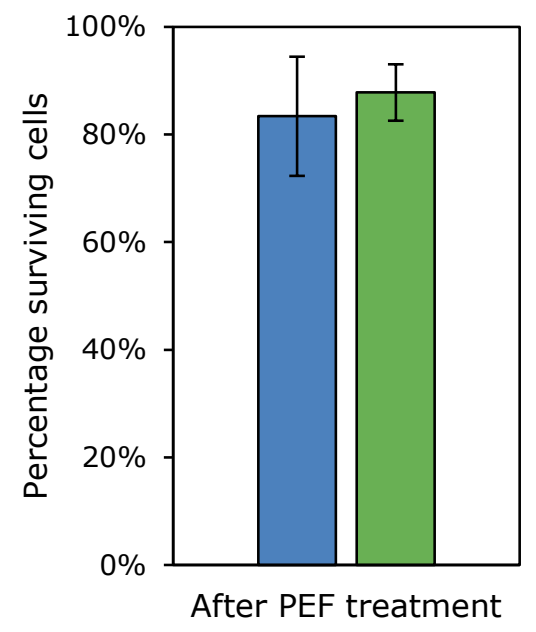

Figure S.4.1 Survival of L. plantarum WCFS1 after PEF treatment in trehalose (blue) and lactose (green) medium. The PEF treatment consisted of 10 pulses of $100 \mu$ s duration at 6.3 $\mathrm{kV} / \mathrm{cm}$. Error bars represent standard deviations of biologically independent replicates, $n \geq 3$.

\section{S.4.2 PI uptake during freezing}
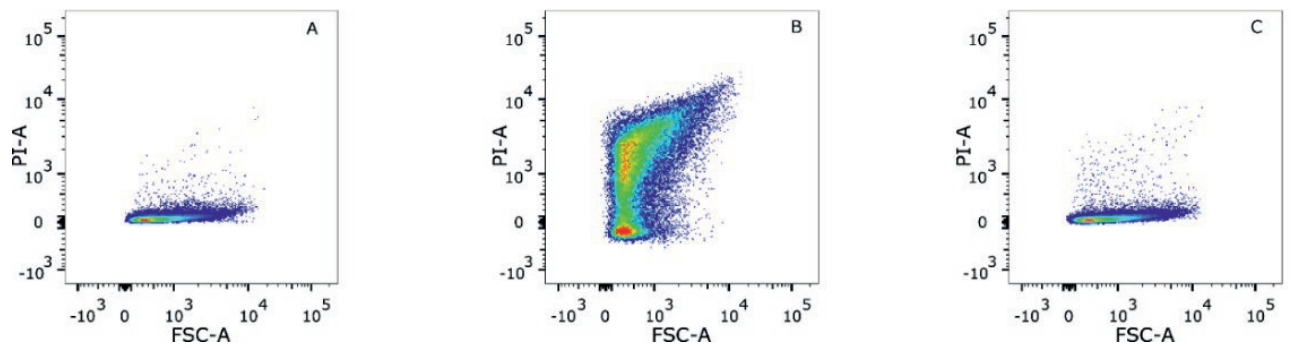

Figure S.4.2 Flow cytometry density plots of L. plantarum WCFS1 cultures stained with propidium iodide (PI). The $y$-axis shows the PI fluorescence intensity and the $x$-axis the forward scatter values. A: Control culture (without PI uptake), B: Example of culture with PI uptake in part of the culture, $C$ : Culture frozen in $-20^{\circ} \mathrm{C}$. Each dot represents a single bacterial cell. The colours represent a density scale from purple/blue (low) to red (high cell density). 


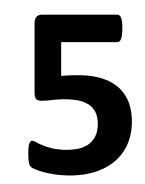

Pulsed electric field pre-treatment for enhanced bacterial survival after drying: Effect of carrier matrix and strain variability 


\begin{abstract}
Previous studies suggest that increased intracellular trehalose concentrations can result in increased robustness of probiotics and starter cultures after processing. We evaluated whether pulsed electric field (PEF) pre-treatment, resulting in increased intracellular trehalose concentrations, can lead to enhanced survival during spray drying and subsequent storage and what the effect of the carrier matrix during drying herein is on survival. The applied PEF pre-treatment resulted in increased survival of Lactobacillus plantarum WCFS1 after spray drying and subsequent storage in reconstituted skim milk (RSM). PEF pretreatment of this strain did not lead to enhanced robustness when spray drying in trehalose or whey protein as a carrier matrix. The same PEF pre-treatment in lactose instead of trehalose electroporation medium resulted also in an increased survival of $L$. plantarum WCFS1 after spray drying. Further analysis of intracellular trehalose and lactose concentrations in this strain revealed that survival after spray drying cannot only be explained by intracellular trehalose and/or lactose concentrations. Notably, optimized PEF pre-treatment of a Lactococcus strain to load the cells with intracellular trehalose, did not provide enhanced survival upon spray drying in RSM. Therefore, strain variability cannot be neglected when designing a process aimed at enhanced survival after processing. Overall, PEF pre-treatment is promising for enhancing survival of bacteria after drying and storage, though further understanding of the applicability is required for industrial application.
\end{abstract}




\subsection{Introduction}

Spray drying and freeze drying can be used to stabilize microorganisms such as probiotics and starter cultures for a prolonged shelf life. These heat sensitive microorganisms are often encapsulated in a carrier matrix consisting of carbohydrates and/or proteins to provide protection during the drying process and subsequent storage of the powders (Broeckx et al., 2017; Huang et al., 2017). Components that are often used as carrier matrix are for example trehalose, lactose, maltodextrin, whey protein or reconstituted skim milk (RSM). Several (micro)organisms that survive well under desiccation stress in nature are known to accumulate protective solutes intracellularly, such as the disaccharide trehalose (Crowe et al., 1992; Zhang and Yan, 2012). Also in processing applications, intracellular trehalose accumulation has been linked to enhanced robustness of several cell types. For example, trehalose has been found intracellularly in Lactobacillus casei and Propionibacterium freudenreichii after pre-conditioning of these bacteria towards osmotic stresses to induce increased survival during subsequent spray drying (Gaucher et al., 2019; Huang et al., 2018). Furthermore, the introduction of trehalose synthesis genes in L. lactis resulted in higher survival during freeze drying (Termont et al., 2006). A different approach to increase intracellular trehalose is pulsed electric field (PEF) treatment in a trehalose medium. Pulsed electric field employs the use of nano- to millisecond high voltage pulses to permeabilize the cellular membrane (Raso et al., 2016). PEF pre-treatment to increase intracellular trehalose was found to lead to increased survival of mammalian and plant cells after subsequent processing (Dovgan et al., 2017; Phoon et al., 2008).

In previous work we showed that pulsed electric field treatment can also be used to increase intracellular trehalose in bacteria, namely in Lactobacillus plantarum WCFS1 (Vaessen et al., 2018). However, this did not lead to enhanced robustness of the PEF pre-treated cultures after drying and subsequent storage of the powders (Vaessen et al., 2019). Remarkably, another recent study with a similar approach did show the opposite, i.e. enhanced robustness of Lactobacillus bulgaricus upon spray drying with PEF pre-treatment to increase intracellular trehalose (Gong et al., 2019). The main differences between these two studies were the microorganism investigated and the carrier matrix during drying. Gong et al. (2019) dried the bacteria in RSM after PEF pre-treatment in trehalose, while our previous study used the same carbohydrate (i.e. trehalose) for both PEF pre-treatment and subsequent drying (Vaessen et al., 2019). We showed that intracellular trehalose concentrations also increased during drying and reconstitution of the powder when 
trehalose was used as the carrier matrix, and this might explain why loading of the cells with trehalose by PEF pre-treatment did not gave a complementary protective effect (Vaessen et al., 2019).

Based on these two recent studies, the use of a PEF pre-treatment to enhance bacterial robustness requires further understanding of the applicability as well as further understanding of the role of intracellular trehalose during drying. Therefore the effect of the carrier matrix as well as the bacterial strain should be further investigated. To study these aspects, we used both spray drying and freeze drying, as these processes are both relevant for industrial drying of bacteria. The aim of this study is to understand the role of intracellular trehalose during drying of lactic acid bacteria; (1) by evaluating the effect of the carrier matrix on survival of Lactobacillus plantarum WCFS1 after spray drying and storage with and without PEF pre-treatment, (2) by comparing the results of Lactobacillus plantarum WCFS1 to another bacterial strain; Lactococcus lactis FM03 and, (3) by comparing accumulation of intracellular trehalose during freeze drying of several lactic acid bacteria in a trehalose drying matrix.

\subsection{Materials and methods}

\subsubsection{Microorganisms and pre-culture}

The microorganisms used in this study were Lactobacillus plantarum WCFS1, Lactobacillus plantarum LMG23545, Lactococcus lactis subsp. lactis bv. diacetylactis FM03 and Lactobacillus acidophilus NCFM (Table 5.1). The first three strains were obtained from the Wageningen Food microbiology strain collection and Lactobacillus acidophilus NCFM was kindly provided by the North Carolina State University, USA.

All Lactobacillus strains were grown on de Man Rogosa and Sharpe (MRS, Merck, USA) medium and L. lactis FM03 on M17 medium with glucose, which is a typical rich medium for Lactococcus strains (M17: BD Difco, USA, glucose monohydrate: Merck, USA). All bacteria were pre-cultured as follows; bacterial strains from the $-80^{\circ} \mathrm{C}$ stock were plated on MRS or $\mathrm{M} 17$ agar plates and incubated under microaerobic conditions at $30^{\circ} \mathrm{C}$ (L. plantarum strains and L. lactis $\mathrm{FMO3}$ ) or $37^{\circ} \mathrm{C}$ (L. acidophilus NCFM) for approximately 70 hours. After incubation these plates were stored at $4^{\circ} \mathrm{C}$ until further use, though for a maximum of 4 days. Subsequently, a single colony was inoculated into $10 \mathrm{ml}$ broth (MRS or M17) and this culture was incubated for $24 \pm 2$ hours without shaking at 30 or $37^{\circ} \mathrm{C}$ respectively. After 
incubation, the culture was transferred into fresh broth in a 1:100 dilution in the required volume for the following experiments and incubated under the same conditions as the previous step. This final pre-culture step was $16-18 \mathrm{hr}$ for the $L$. plantarum strains and $L$. lactis FM03, and 20-22 hr for L. acidophilus NCFM to obtain cultures in the stationary growth phase.

Table 5.1 Bacterial strains used in this study and their origin

\begin{tabular}{lll}
\hline Bacterial strain & Isolation & Reference \\
\hline $\begin{array}{l}\text { Lactobacillus plantarum } \\
\text { WCFS1 }\end{array}$ & $\begin{array}{l}\text { Originally isolated from } \\
\text { human saliva }\end{array}$ & (Kleerebezem et al., 2003) \\
\hline $\begin{array}{l}\text { Lactobacillus plantarum } \\
\text { LMG23545 }\end{array}$ & $\begin{array}{l}\text { Originally isolated from } \\
\text { healthy adult faeces }\end{array}$ & (Aryani et al., 2016) \\
\hline $\begin{array}{l}\text { Lactococcus lactis subsp. } \\
\text { lactis biovar diacetylactis }\end{array}$ & $\begin{array}{l}\text { Isolated from 10-week-old } \\
\text { FM03 }\end{array}$ & (vans $\varnothing$ cheese \\
\hline Lactobacillus acidophilus & Human intestinal isolate & (Duong et al., 2006) \\
NCFM & & \\
\hline
\end{tabular}

For every experiment the pre-cultured bacteria were centrifuged for 10 minutes at 13,500 $x g$ and subsequently the pellet was suspended in washing buffer and centrifuged again, in the same way as described in Vaessen et al. (2018). During this step L. lactis FM03 cultures were five times concentrated and $L$. acidophilus cultures two times to have cultures with a similar $\mathrm{OD}_{600}$ as L. plantarum cultures. Subsequently, the bacterial pellet was resuspended in the trehalose electroporation medium containing $0.3 \mathrm{M}$ trehalose dihydrate (SigmaAldrich, USA) or lactose electroporation medium containing 0.3 $\mathrm{M}$ lactose monohydrate (VWR, USA) (for rest of composition and composition of the washing buffer, see: (Vaessen et al., 2018)). The culture in electroporation medium had a conductivity of $0.15 \mathrm{~S} / \mathrm{m}$ at room temperature.

\subsubsection{Pulsed electric field treatment}

Lactobacillus plantarum WCFS1 and Lactococcus lactis FM03 were PEF treated in an IXL batch PEF system with custom made treatment chamber as extensively described in our 
previous publication (Vaessen et al., 2019). This treatment chamber consists of two parallel titanium electrodes with a distance of $0.3 \mathrm{~mm}$ in which a volume of approximately $13 \mathrm{~mL}$ could be PEF treated. The PEF treatment for L. plantarum WCFS1 consisted of 10 square wave pulses of $6.3 \mathrm{kV} / \mathrm{cm}$ with a pulse duration of $100 \mu \mathrm{s}$ and pulse interval of $5 \mathrm{~s}$. The PEF treatment for L. lactis FM03 consisted of 50 pulses, with all other settings the same as for L. plantarum WCFS1. The water bath attached to the treatment chamber was set at a temperature of $21^{\circ} \mathrm{C}$. For every experiment, part of the bacterial culture was kept as a control (without PEF treatment). PEF treated and control samples were used for survival analysis and intracellular trehalose measurements.

\subsubsection{Spray drying and storage tests}

Carrier matrices of $20 \%(\mathrm{w} / \mathrm{w})$ solids concentration were prepared by combining demineralized water with maltodextrin DE19 (MD19, Roquette, France), reconstituted skim milk (RSM, Arla Food Ingredients, Denmark), whey protein (BiPro ${ }^{\circ}$, Davisco, Switzerland) or trehalose (Merck, USA). Five $\mathrm{mL}$ of bacterial culture in trehalose or lactose electroporation medium (control and PEF-treated) were added to $50 \mathrm{~mL}$ of the prepared carrier matrices. Samples were taken for survival analysis before spray drying. The culture in carrier matrix was subsequently spray dried in a Büchi B-290 laboratory spray dryer (Büchi Labortechnik $A G$, Switzerland) with an inlet temperature of $140^{\circ} \mathrm{C}$. The flow rate was adjusted to obtain an outlet temperature of $80 \pm 1^{\circ} \mathrm{C}$. After spray drying, the powder was collected and part of the powder was distributed in samples of $200 \pm 3 \mathrm{mg}$, which were subsequently used for survival analyses and storage tests. Additionally, the moisture content was measured using a moisture analyser (Sartorius, Germany), and was 3-5\% (w/w) for all experiments. For survival analysis during storage, the samples were stored at room temperature $\left(21 \pm 2^{\circ} \mathrm{C}\right)$ in desiccators containing an oversaturated potassium acetate solution $\left(a_{w}=0.23\right)$. Samples for survival analysis were taken approximately every 21 days.

\subsubsection{Freeze drying experiments}

Bacterial cultures in the trehalose electroporation medium were distributed into several 1 $\mathrm{ml}$ samples and subsequently frozen at $-20^{\circ} \mathrm{C}$ and freeze dried in a Christ Epsilon 2-6D freeze dryer (Martin Christ, Germany), in the same way as described in previous research (Vaessen et al., 2019). Samples for survival and intracellular trehalose analyses were taken before freezing, after freezing and after freeze drying. 


\subsubsection{Survival assessment}

Samples for survival analysis were decimally diluted in peptone physiological salt solution (PPS, Tritium Microbiologie, the Netherlands). Dilution series were made at least in duplicate and the appropriate dilutions were subsequently plated on MRS or M17 agar in duplicate. Plates were incubated microaerobically at 30 or $37^{\circ} \mathrm{C}$ respectively for $2-4$ days. After incubation, colonies were counted and survival was calculated by dividing the $\mathrm{cfu} / \mathrm{ml}$ of the sample after processing (after PEF, freezing or drying) by the $\mathrm{cfu} / \mathrm{ml}$ of the sample before processing. Dried samples were first rehydrated in $10 \mathrm{~mL}$ PPS, resulting in the -1 dilution and subsequently further diluted and plated as described above.

\subsubsection{Intracellular trehalose measurements}

Intracellular trehalose extraction and subsequent measurement with HPAEC analysis was performed according to the method described in Vaessen et al. (2019). Briefly, extracellular trehalose was washed away with three washing steps in phosphate buffered saline (PBS). Subsequently, the cell pellet was suspended in milliQ water and homogenized in a bead beater to extract intracellular trehalose. Trehalose concentrations in these samples were measured using high pressure anion exchange chromatography with PAD detection.

\subsubsection{Experimental set-up}

All experiments were carried out at least in duplicate, with each replicate being biologically independent, pre-cultured on another day. Additionally, measurement replicates were taken into account for all analyses. Significance was tested with a Student's $t$-test, using a P-value of 0.05 .

\subsection{Results and discussion}

\subsubsection{Chosen carrier matrix during drying affects added value of PEF pre- treatment}

The results of two recent studies on survival of Lactobacilli after drying with a pulsed electric field pre-treatment leading to increased intracellular trehalose concentrations seem to contradict each other (Gong et al., 2019; Vaessen et al., 2019). One of the major differences between these two studies is the carrier matrix that was used after the PEF treatment. This difference suggests that the type of carrier matrix might play a critical role. Therefore, we dried L. plantarum WCFS1 with and without PEF pre-treatment in trehalose electroporation 
medium in several carrier matrices, namely, maltodextrin DE19, reconstituted skim milk (RSM), whey protein and trehalose. The applied PEF pre-treatment resulted in an increase in intracellular trehalose and a survival of approximately $80 \%$ (Fig. 5.1).

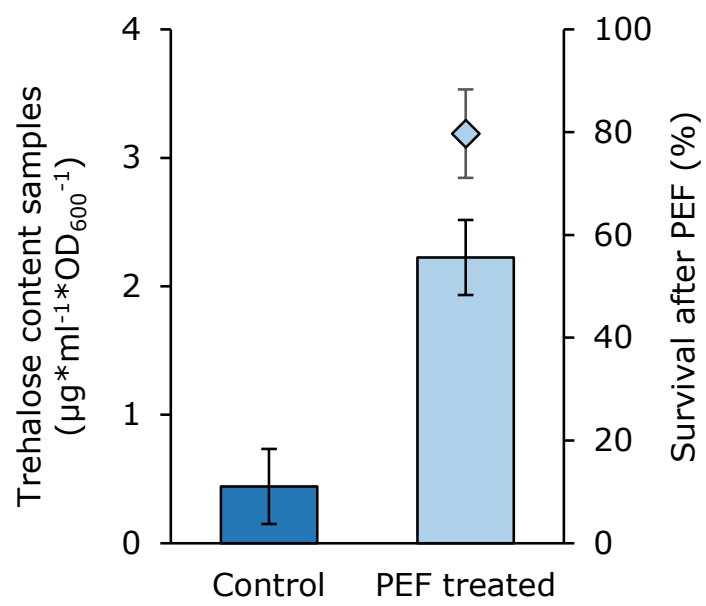

Figure 5.1 Intracellular trehalose content (bars) and survival (blue diamond) after PEF treatment of Lactobacillus plantarum WCFS1 in trehalose electroporation medium. Intracellular trehalose is presented as trehalose concentration of the cell extract per $O D$ value of the culture. Error bars represent standard deviations of independent replicates $(n=9)$.

Our results indeed confirmed that when drying in trehalose, no difference in survival of cells with and without PEF pre-treatment was found (Fig. 5.2), similar to what was observed after freeze drying in our previous study (Vaessen et al., 2019). Interestingly, PEF pre-treated cultures showed significantly increased survival after spray drying when performing the PEF treatment in trehalose electroporation medium and subsequently spray drying the culture in reconstituted skim milk ( $P=0.02)$ (Fig. 5.2). The average survival of the $P E F$ treated culture was doubled compared to the culture without PEF pre-treatment. This difference indicates that PEF pre-treatment is a promising strategy for increasing survival after spray drying, although the carrier matrix plays an important role in this effect. Furthermore, these results are in agreement with the studies of Gong et al. (2019) and Termont et al. (2006). In the latter study the intracellular trehalose concentrations in L. lactis were increased by a genetic modification approach, and increased intracellular trehalose concentrations led to enhanced robustness during freeze drying in RSM and subsequent incubation in gastric juice. 


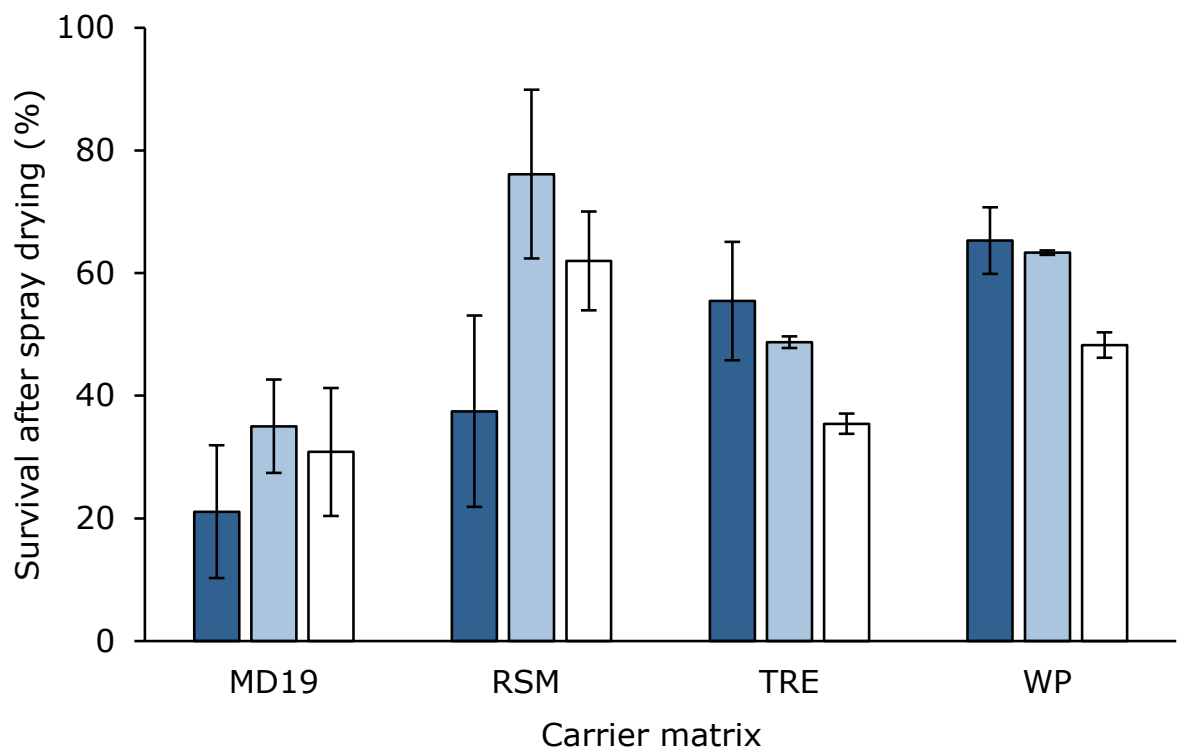

Figure 5.2 Survival of L. plantarum WCFS1 after spray drying in different carrier matrices with and without PEF pre-treatment leading to increased intracellular trehalose. Different colours represent survival after spray drying of control cultures (blue), PEF pre-treated cultures (light blue) and PEF pre-treated cultures corrected for PEF survival (white). Error bars indicate standard deviations of biologically independent replicates $(n \geq 2)$.

The spray drying experiments with maltodextrin DE19 as carrier matrix also showed an increasing trend in average survival of the PEF pre-treated culture compared to the control, though not as clear as for RSM (Fig. 5.2). For spray drying in whey protein no difference was observed between the survival of the PEF pre-treated culture and the control culture. These findings raise the question why PEF pre-treatment, leading to increased intracellular trehalose (Fig. 5.1), only has a beneficial effect when a specific carrier matrix is used during drying. For trehalose as carrier matrix we could explain this by the increase in intracellular trehalose during drying processes itself when this sugar is used as a carrier matrix (Vaessen et al., 2019). However, for whey proteins a similar effect is not expected based on the much larger size of these molecules compared to trehalose. Reconstituted skim milk is a mixture of proteins (mainly casein and whey protein), lactose and minerals. Why PEF pre-treatment leads to enhanced survival specifically in this matrix remains to be elucidated. 
In the results presented in the light blue bars in Fig. 5.2 the decrease in survival during the PEF treatment itself was not taken into account. Survival of $L$. plantarum WCFS1 after a PEF treatment with ten pulses at $6.3 \mathrm{kV} / \mathrm{cm}$ was approximately $80 \%$ (Fig. 5.1). When taking into account this survival, the differences in survival after spray drying between the PEF pretreated and control cultures become smaller (white bars). For trehalose and whey protein as carrier matrices even a decrease in overall survival was observed when taking the survival of the PEF treatment into account. The average survival in the overall process increased approximately from 37 to $62 \%$ in RSM as carrier matrix when PEF pre-treatment was applied. This increase is similar to what Gong et al. (2019) observed, they found a maximum survival increase over the whole process from 38 to $61 \%$ upon applying PEF pre-treatment. Summarizing, these results confirmed our hypothesis that the efficacy of a PEF pretreatment, leading to increased intracellular trehalose (Fig. 5.1), depends on the carrier matrix that is used.

Next to PEF pre-treatment, also the carrier matrix affected the survival after spray drying. Drying the culture (without PEF pre-treatment) in trehalose and whey protein resulted in the highest survival under these drying conditions, followed by RSM and maltodextrin DE19. Higher survival of trehalose after spray drying compared to maltodextrin is in line with previous research with trehalose and maltodextrin DE16 (Perdana et al., 2014b). Not only the survival differed between these drying matrices, also the final morphology of the dried powder particles (Fig. 5.3). Reconstituted skim milk and maltodextrin particles were wrinkled, and trehalose and whey protein particles had a smooth surface. Compared to the RSM particles, the maltodextrin particles had more smaller wrinkles (Fig. 5.3). The relationship between carrier matrix and survival is very complex and strongly depends on the physicochemical properties of the carrier matrix. These properties influence survival not only in a direct way, e.g. via glass transition or water replacement, but also indirectly via morphology development and related drying kinetics of the droplets. (Khem et al., 2015; Siemons et al., 2020b; Wang et al., 2016). The lower survival of L. plantarum WCFS1 in wrinkled particles (maltodextrin and RSM) compared to particles with a smooth surface (whey protein and trehalose) is in line with observations after single droplet drying by Siemons et al. (2021). 

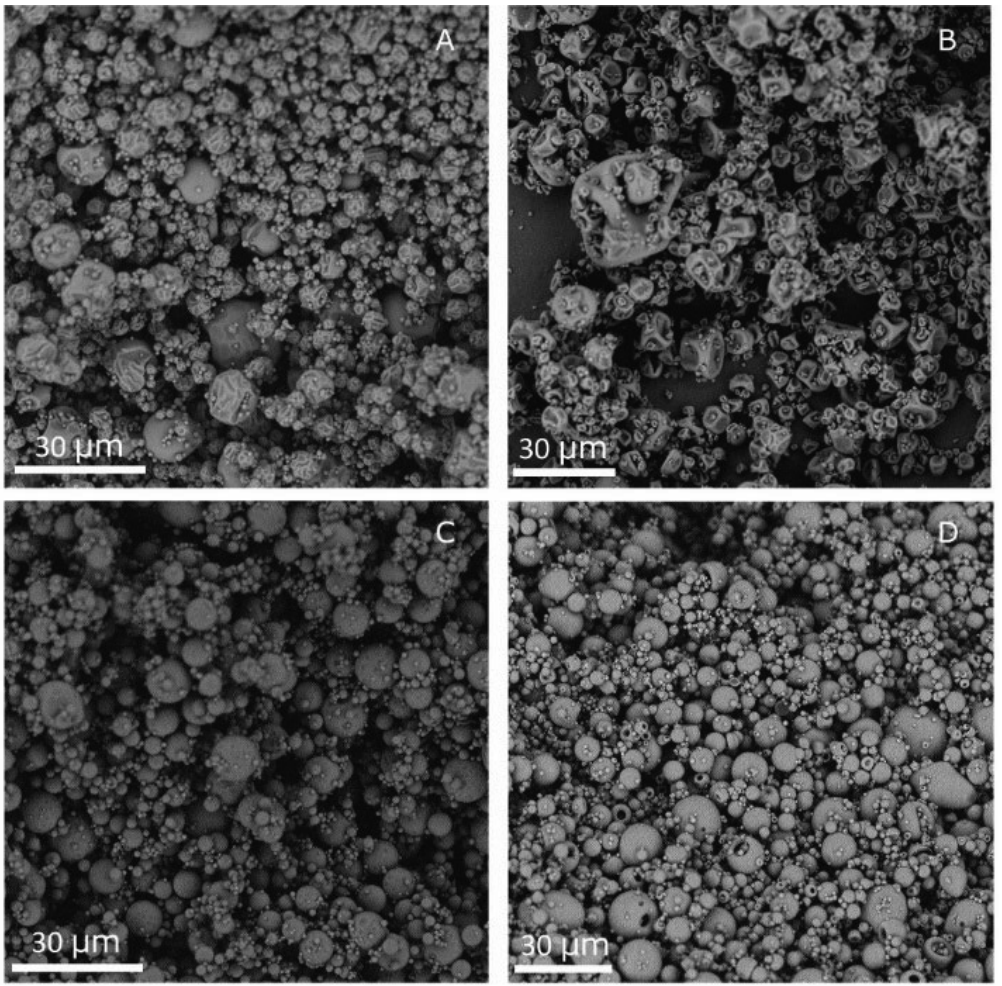

Figure 5.3 SEM images showing the morphology of dried powder particles after spray drying with an inlet temperature of $140^{\circ} \mathrm{C}$ and outlet temperature of $80^{\circ} \mathrm{C}$. The panels show maltodextrin $D E 19(A)$, reconstituted skim milk $(B)$, trehalose $(C)$ and whey protein $(D)$.

\subsubsection{Survival during storage after drying}

In addition to survival during the spray drying process itself, bacterial survival during storage is important for prolonged shelf life of dried probiotics and starter cultures. Therefore we also monitored survival of spray dried L. plantarum WCFS1 cultures in RSM and trehalose with and without PEF pre-treatment for a period of 15 weeks (Fig. 5.4). Interestingly, the PEF pre-treated cultures that were spray dried in RSM survived better during storage compared to cultures without PEF pre-treatment (Fig. 5.4A). The CFU counts of the PEF pretreated cultures remained approximately 0.5 log higher (i.e. factor 3 ) than the control cultures at every time point. This difference shows that the PEF pre-treatment not only contributes to increased survival after drying in RSM, but also during subsequent storage. 

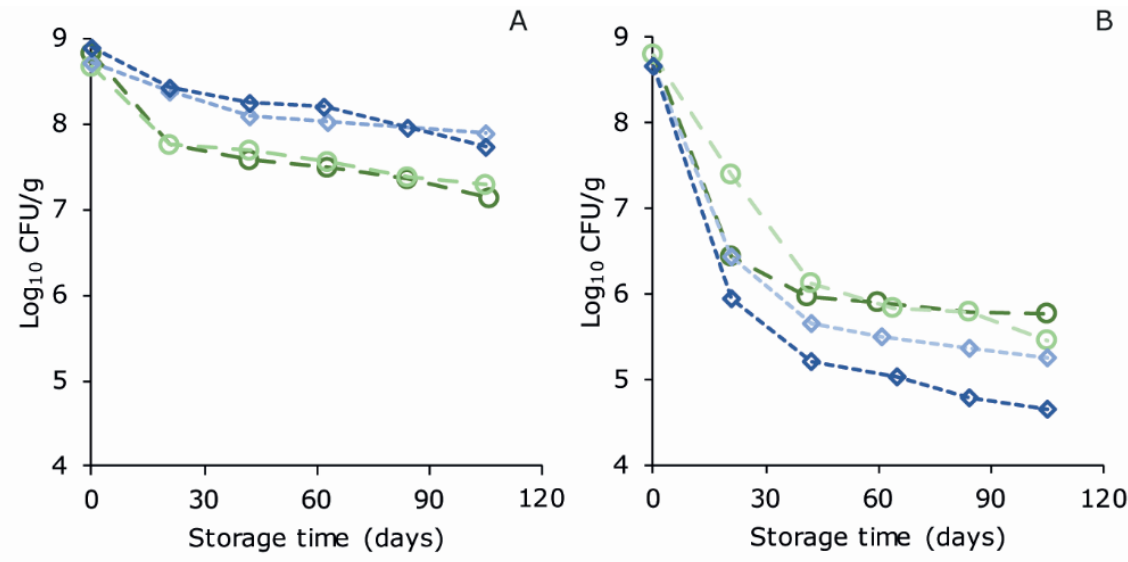

Figure 5.4 Survival of spray dried L. plantarum WCFS1 during storage (room temperature, $\left.a_{w}=0.23\right)$. Control (green circles) and PEF pre-treated (blue diamonds) cultures were spray dried in reconstituted skim milk (A) and trehalose (B). Each experiment has been performed with independent duplicates, as presented by the slightly different colour intensities.

Such a difference was not observed for cultures dried in trehalose (Fig. 5.4B). Both the PEF pre-treated and control cultures decreased in survival over time, and the PEF pre-treatment even seemed to negatively affect survival. During 15 weeks of storage the cultures dried in trehalose reduced much more in viability compared to the cultures dried in RSM. This difference indicates that RSM is the best matrix of these two for protection of $L$. plantarum WCFS1 during storage of the spray dried powders. This protective effect of RSM during storage is in line with results of Ananta et al. (2005), who observed a very limited decrease in survival during storage of spray dried L. rhamnosus GG with RSM as a carrier. Furthermore, Broeckx et al. (2017) found that in addition to the presence of carbohydrates, the presence of phosphates in the carrier matrix contributes to better survival of $L$. rhamnosus GG during storage in these powders. Soukoulis et al. (2014) found that a combination of skim milk powder and maltodextrin provided better storage protection for L. acidophilus than a combination of whey protein and maltodextrin or sodium caseinate and maltodextrin. Additionally, several studies describe protective effects of the presence of calcium in the carrier matrix (Zheng et al., 2016, 2015). As stated before, RSM is a mixture of proteins, lactose and minerals, among which calcium phosphates, and the combination of these components apparently results in a better storage stability than the presence of only a carbohydrate, i.e. trehalose. 


\subsubsection{PEF pre-treatment in lactose}

We showed that PEF pre-treatment in trehalose can increase survival of L. plantarum WCFS1 during spray drying in RSM. This raises the question whether this would also be the case when the PEF pre-treatment is done in another carbohydrate. PEF pre-treatment in lactose electroporation medium resulted in increased intracellular lactose concentrations in L. plantarum WCFS1 (Fig. 5.5B), similar to our previous study (Vaessen et al., 2019). We spray dried these control and PEF pre-treated cultures in RSM medium. Interestingly, the survival of cultures that were PEF pre-treated in lactose significantly increased compared to the control cultures without PEF pre-treatment (Fig. 5.5A). In this case, all PEF treated cells survived spray drying in RSM, even more compared to when PEF pre-treatment in trehalose was applied (see Fig.5.2).
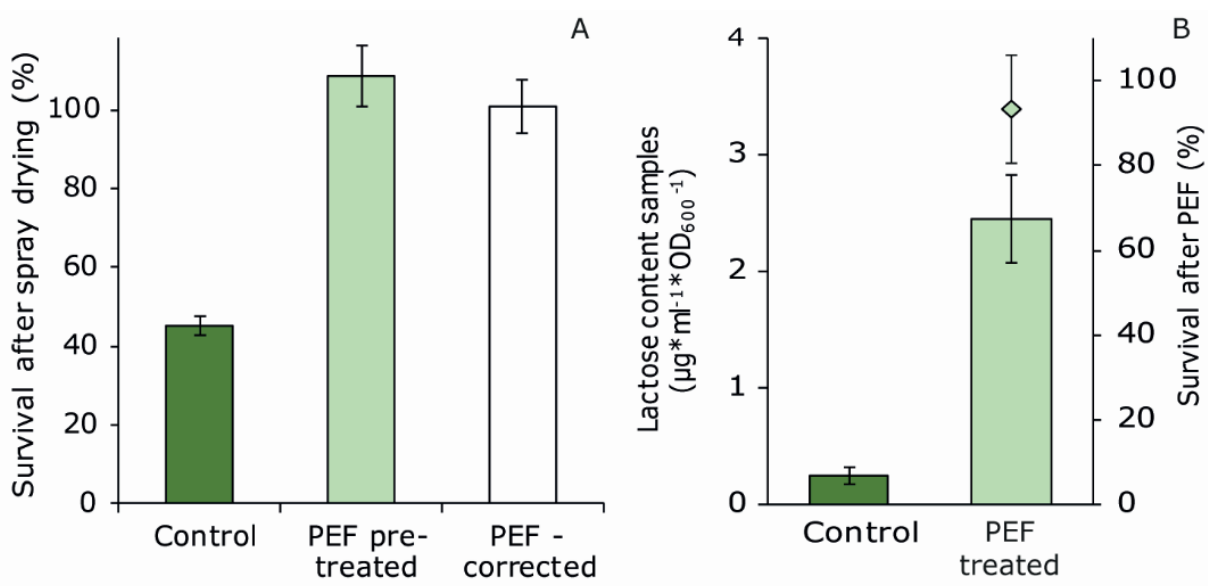

Figure 5.5 Survival of L. plantarum WCSF1 in RSM with and without PEF pre-treatment in lactose electroporation medium (A). And intracellular lactose content (bars) and survival (green diamond) of L. plantarum WCFS1 after PEF treatment in lactose (B). Intracellular lactose is presented as lactose concentration of the cell extract per OD-value of the culture. Error bars indicate standard deviations of independent duplicates.

Not only intracellular lactose was detected in the PEF pre-treated cells with lactose, also trehalose was found intracellularly, while no trehalose was added to the electroporation or drying solution (Table 5.2). Also in our previous study, we found intracellular trehalose in cultures that were suspended in lactose electroporation medium (Vaessen et al., 2019). L. plantarum WCFS1 contains relatively high intracellular trehalose concentrations directly after growth in MRS medium (data not shown). These concentrations decreased when 
suspending the culture in trehalose electroporation medium, but remained high when suspending the culture in lactose electroporation medium. As discussed in Vaessen et al. (2019), this may be explained by a phenomenon called catabolite repression, which is a mechanism by which the catabolism of a specific carbohydrate is repressed when another preferred carbohydrate is present (Görke and Stülke, 2008; Siragusa et al., 2014) . Though further research is required to unravel the exact biological mechanisms involved in this observation.

Table 5.2 Intracellular trehalose and lactose concentrations in L. plantarum WCFS1 suspended in trehalose or lactose electroporation medium with and without PEF. Results are presented as average \pm standard deviation. $<0.05$ means below the detection limit in this sample.

\begin{tabular}{llll}
\hline $\begin{array}{l}\text { Carbohydrate in } \\
\text { electroporation } \\
\text { medium }\end{array}$ & Sample & $\begin{array}{l}\text { Trehalose content } \\
\text { sample } \\
\left(\mu \mathrm{g}^{*} \mathrm{ml}^{-1} * \mathrm{OD}_{600}{ }^{-1}\right)\end{array}$ & $\begin{array}{l}\text { Lactose content } \\
\text { sample } \\
\left(\mu \mathrm{g}^{*} \mathrm{ml}^{-1} *^{*} \mathrm{OD}_{600}{ }^{-1}\right)\end{array}$ \\
\hline Trehalose & $\begin{array}{l}\text { Control } \\
(\mathrm{n}=9)\end{array}$ & $0.4 \pm 0.3^{*}$ & $<0.05$ \\
\hline Trehalose & $\mathrm{PEF}$ & $2.2 \pm 0.3^{*}$ & $<0.05$ \\
\hline Lactose & $(\mathrm{n}=9)$ & & $0.2 \pm 0.1^{*}$ \\
\hline Lactose & $\begin{array}{l}\text { Control } \\
(n=2)\end{array}$ & $6.3 \pm 0.3$ & $2.5 \pm 0.4^{*}$ \\
\hline & PEF & $3.4 \pm 0.2$ & \\
\hline
\end{tabular}

*These results are also presented in Figure 5.1 and 5.5

The presence of intracellular trehalose alone is apparently not enough to provide protection during drying. When comparing the survival after spray drying in RSM of the control culture in trehalose electroporation medium (with low intracellular trehalose content) to the control culture in lactose electroporation medium (with high intracellular trehalose content), the survival was in both cases approximately 40\%. PEF pre-treatment, leading to increased intracellular trehalose or lactose concentrations respectively, resulted in both cases in an increased survival after spray drying (Fig. 5.2 and 5.5A). This might suggest that PEF treatment also affects cells in another way that enhances their robustness towards stresses. However, in that case we would also expect a beneficial effect of the PEF pre- 
treatment upon subsequent drying in other carrier matrices, including trehalose, which was not the case (Fig. 5.2). Some studies indeed suggested that electric fields affect other cellular responses in Lactobacilli (Loghavi et al., 2008; Najim and Aryana, 2013). For example, Najim et al. (2013) observed an increase in acid tolerance, protease activity and growth of $L$. rhamnosus and L. bulgaricus upon mild PEF treatment. Overall, our results show that survival of bacteria after drying is multifactorial and cannot be explained by only the accumulation of intracellular trehalose and/or lactose.

\subsubsection{PEF pre-treatment for Lactococcus lactis}

PEF pre-treatment in trehalose and subsequent drying in RSM was also carried out for Lactococcus lactis FM03 to extend our insights obtained from L. plantarum WCFS1. This strain was chosen because Lactococcus lactis is not only a different species than Lactobacillus plantarum, but also a very relevant lactic acid bacteria because it is a commonly used starter culture (Leroy and De Vuyst, 2004). Additionally, this Lactococcus strain is less heat robust than Lactobacillus plantarum WCFS1 (supplementary material S.5.1). The optimized PEF treatment conditions to increase intracellular trehalose concentrations for this strain differed from the conditions applied for L. plantarum WCFS1; for L. lactis FM03 the PEF treatment was 50 pulses of $100 \mu \mathrm{s}$ at $6.3 \mathrm{kV} / \mathrm{cm}$ (Fig. 5.6B). Based on the intracellular concentrations that were corrected for the $\mathrm{OD}_{600}$ of the culture, the intracellular trehalose concentration after PEF in L. lactis FM03 was in the same range as for L. plantarum WCFS1 (Fig. 5.1 \& 5.6), although the variation between the replicates was higher for L. lactis FM03. Also the survival of L. lactis FM03 after the applied PEF treatment remained high, approximately 90\% (Fig 5.6B). L. lactis FM03 was dried in RSM, in the same way as was done for L. plantarum WCFS1. However, despite an increase in intracellular trehalose was observed after PEF treatment, no increase in survival was observed for the PEF pre-treated culture compared to the culture without PEF pre-treatment (Fig 5.6A). This result was not expected based on the results obtained for $L$. plantarum WCFS1, the results of Gong et al. (2019) for L. bulgaricus and the results of Termont et al. (2006) for another Lactococcus lactis strain. Survival of L. lactis FM03 without PEF pre-treatment was approximately $16 \%$, which is lower than survival of L. plantarum WCFS1 after spray drying, using the same conditions ( 37\%). The lower survival for L. lactis FM03 compared to $L$. plantarum WCFS1 after spray drying is in agreement with the heat robustness of these strains (supplementary material S1). Overall, these experiments with L. lactis FM03 showed 
that PEF pre-treatment leading to increased intracellular trehalose concentrations might not lead to the same protective effects towards drying for different bacterial strains.
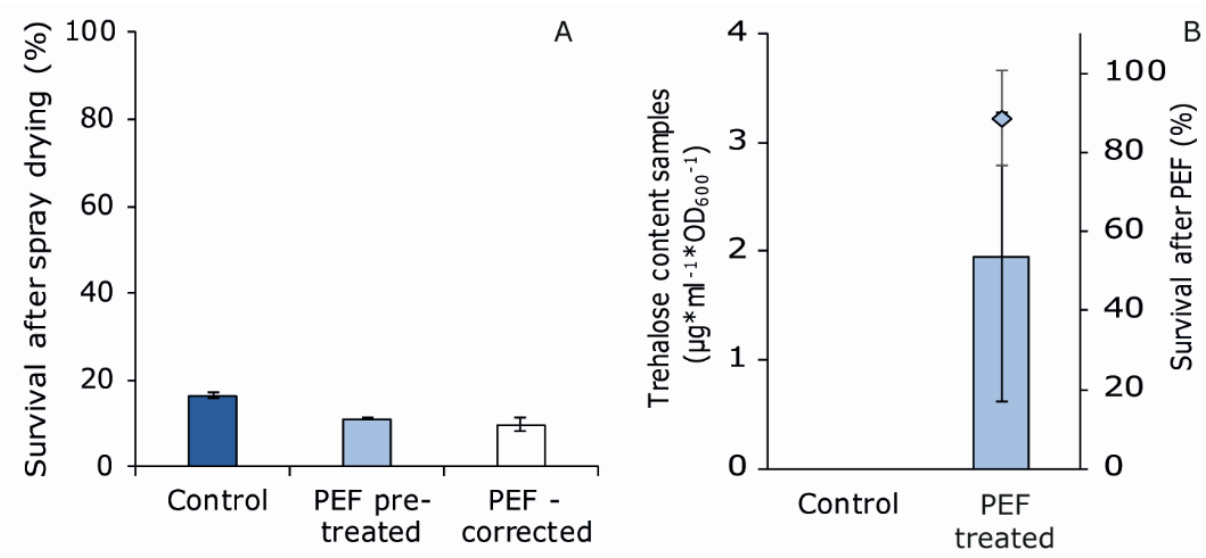

Figure 5.6 Survival of L. lactis FMO3 after spray drying in RSM with and without PEF pretreatment in trehalose (A) and intracellular trehalose and survival after PEF treatment (B). Error bars indicate standard deviations of independent duplicates.

This observation triggered the question how variable are the intracellular trehalose concentrations during drying of different bacterial strains. For these experiments we have chosen freeze drying as the drying method, because of experimental convenience and the possibility of not only evaluating the intracellular concentrations after drying, but also after the initial freezing step. This could provide extra information on intracellular trehalose concentrations during drying in a trehalose medium after both freezing and freeze drying in several lactic acid bacteria.

\subsubsection{Intracellular trehalose in several strains during freeze drying}

Intracellular trehalose concentrations were monitored during the different steps of the freeze drying process in trehalose medium for Lactobacillus plantarum WCFS1, Lactococcus lactis FM03, Lactobacillus plantarum LMG23545 and Lactobacillus acidophilus NCFM. During our earlier work we observed that intracellular trehalose increased in Lactobacillus plantarum WCFS1 after the freezing step before freeze drying (Vaessen et al., 2019). Interestingly, this is not found for the other bacteria tested in this study (Fig. 5.7), while the experimental set-up was the same. The intracellular trehalose results for the different strains are presented as concentrations in the sample divided by the $\mathrm{OD}_{600}$ of the culture in 
order to correct for cell sizes and amounts of cells to be able to compare the different strains.

In L. lactis FM03 intracellular trehalose was very low before and after freezing, but after freeze drying and reconstitution of the powder it was much higher. For L. plantarum LMG23545 and L. acidophilus NCFM there was already a substantial concentration of intracellular trehalose present in the cells before the freeze drying process and intracellular trehalose increased for both strains after freeze drying, though for L. acidophilus NCFM this increase was not significant. This smaller increase could also be related to the lower survival of this strain after freeze drying (Fig 5.7B). Although we are not completely sure whether the intracellular trehalose that we measured is only present in the surviving cells, it is likely that the intracellular trehalose of dead/damaged cells is washed away during the washing steps before the trehalose extraction. Overall, these data indicate that the presence of intracellular trehalose before the freezing treatment differs between bacterial strains. Also the moment in the process where an increase in intracellular trehalose is observed, differs between the evaluated strains; e.g. after freezing in L. plantarum WCFS1 and after freeze drying in the other strains.
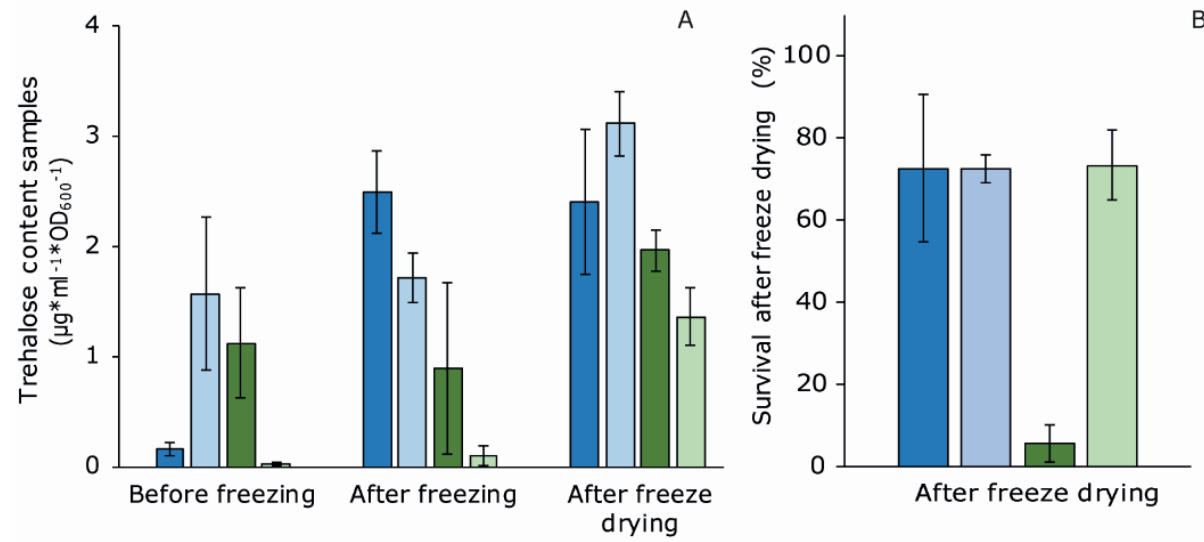

Figure 5.7 Intracellular trehalose content and survival of L. plantarum WCFS1 (blue) , L. plantarum LMG23545 (light blue), L. acidophilus NCFM (green) L. lactis FM03 (light green) after freeze drying in trehalose. Intracellular trehalose content is presented in in $\mathrm{ug} / \mathrm{ml}$ sample divided by the OD600 of the culture to correct for amounts of cells present in the sample. Error bars represent standard deviations of biologically independent duplicates. Data for L. plantarum WCFS1 were adapted from Vaessen et al. (2019). 
The high intracellular trehalose contents already present before any treatment in some bacterial strains make it questionable whether a PEF pre-treatment to increase intracellular trehalose would be beneficial for these bacteria. There seems no direct relation between the intracellular trehalose present before the freeze drying process and subsequent robustness as illustrated by $L$. acidophilus NCFM. This strain had already trehalose intracellular before freeze drying treatment, however this strain was the least robust of the tested strains during freeze drying (Fig. 5.7B).

Strain differences in robustness towards drying processes are very common and a challenge for designing general guidelines for drying of bacteria (Dijkstra et al., 2014; Lian et al., 2002; Mille et al., 2004). Variability in bacterial robustness during processing has also been studied much more for pathogens and spoilage microorganisms, and these studies showed that variability among strains of the same species can be as significant as among strains of different species (Alvarenga et al., 2018; den Besten et al., 2018).

\subsection{Conclusion}

The effectivity of a pulsed electric field pre-treatment to increase survival after spray drying of L. plantarum WCFS1 and subsequent powder storage depended on the carrier matrix that was used during drying. We found only an added value of the PEF pre-treatment in trehalose electroporation medium when RSM was used as the carrier matrix. The exact mechanism by which a PEF pre-treatment resulted in increased survival in this matrix remains to be elucidated since it cannot be explained by only the intracellular carbohydrate concentrations upon PEF treatment. Our findings for other lactic acid bacteria show that strain variability cannot be neglected when developing a process aiming at enhanced bacterial survival after processing and that further understanding of the complex interplay between intracellular protective solutes and the extracellular carrier matrix is necessary. 


\section{Supplementary information}

\section{S.5.1. Heat robustness of $L$. plantarum WCFS1 and L. lactis FM03}

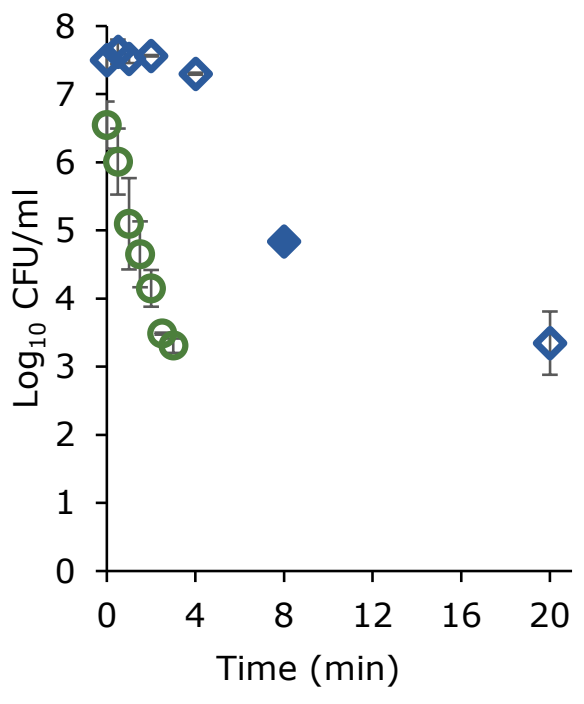

Figure S.5.1 Inactivation of L. plantarum WCFS1 (blue diamonds) and L. lactis FM03 (green circles) in a water bath at $58^{\circ} \mathrm{C}$, according to the method described in Aryani et al. (2016). Both cultures were pre-cultured as described in the materials and methods section, except for the last step, which was a 1:10 dilution in this case instead of 1:100 in all other experiments. Error bars represent standard deviations of independent duplicates (closed symbol: $n=1)$. 



\section{6}

\section{Protective effect of carrier matrices on survival of Lactobacillus plantarum WCFS1 during single droplet drying explained by particle morphology development}

This chapter has been published as Siemons, I. ${ }^{1}$, Vaessen, E. M. J. ${ }^{1}$, Oosterbaan van Peski, S. E., Boom, R. M., \& Schutyser, M. A. I. (2021) Protective effect of carrier matrices on survival of Lactobacillus plantarum WCFS1 during single droplet drying explained by particle morphology development. Journal of Food Engineering, 292, 110263 


\section{Abstract}

Spray drying can be used to manufacture stable powder ingredients with living bacteria. Usually a carrier matrix consisting of carbohydrates and/or proteins is used to increase survival of the bacteria during drying. However, it is not exactly known why some matrices provide better protection than others. Depending on the carrier matrix and the drying conditions applied we also know that different powder particle morphologies are obtained. In this study we employed sessile single droplet drying to investigate the relation between the particle morphology and the survival of Lactobacillus plantarum WCFS1 after drying. Different drying matrices with varying physicochemical properties were selected that resulted in smooth, hollow or dented particle morphologies. A clear correlation was observed between the observed particle morphologies and the viabilities after drying. Highest survival was obtained for dense and smooth particles, whereas lowest survival was obtained for dented particles. The relation between the morphology and survival is likely to be rooted in a complex interplay between matrix properties and drying dynamics, where a skin that hinders evaporation leads to the highest inactivation. The identified correlation between morphology development and bacterial survival will be important to identify the mechanisms of the inactivation of bacteria during drying. 


\subsection{Introduction}

Lactic acid bacteria are widely used in the production of food as starter cultures or probiotics. These bacterial food ingredients are often dried and stored in the form of a powder to increase their shelf life (Broeckx et al., 2016; Huang et al., 2017; Peighambardoust et al., 2011). Freeze and spray drying are the main drying techniques that are used to industrially dry probiotics and starter cultures. Although during freeze drying the heat sensitive microorganisms are less exposed to heat as is the case during spray drying, spray drying is an interesting alternative since the drying time is shorter and the energy consumption is much smaller compared to freeze drying (Huang et al., 2017; Santivarangkna et al., 2007).

During spray drying, considerable inactivation of bacteria occurs due to thermal and dehydration stresses (Liu et al., 2018; Perdana et al., 2013). Therefore, bacteria are commonly dried in a protective matrix, consisting of for example carbohydrates, proteins or reconstituted skim milk to enhance their viability after drying (Broeckx et al., 2017; Perdana et al., 2014b). The protective effect of these drying matrices, especially for sugars, is often explained by their ability to form a glassy matrix in which the cells are embedded and/or they may depress a phase change of the cellular membrane from liquid crystalline to gel phase (Broeckx et al., 2016; Leslie et al., 1995; Perdana et al., 2014b). Additionally, the viability is also influenced by drying kinetics of the matrices, leading to a specific temperature-moisture content history of the droplets during drying (Ghandi et al., 2012a; Khem et al., 2015). In general, survival decreases considerably when the product is exposed to higher temperatures at higher moisture contents for a longer time (Liu et al., 2018; Perdana et al., 2013). The fact that some strategies lead to higher viability after spray drying shows that it is possible to increase the survival, though there is still a lack of insight in the reasons why some drying matrices result in better protection during spray drying compared to others.

Spray drying can yield entirely distinct particle morphologies depending on the carrier matrix and the applied drying conditions (e.g. smooth, hollow, dented) (Both et al., 2018; Siemons et al., 2020a). Hitherto, particle morphology development during spray drying has been mainly studied with respect to physical powder properties such as bulk density and particle size (Both et al., 2018; Sadek et al., 2014; Schutyser et al., 2019). Some studies suggested that the development of the skin and the overall particle morphology during spray drying may also affect bacterial survival (Ghandi et al., 2012b; Khem et al., 2016, 2015; Wang et al., 2016). However, it is not known whether the particle morphology and bacterial survival can be linked. We here therefore hypothesize that the particle morphology as 
influenced by the properties of the carrier materials and drying conditions also has a specific effect on the survival of bacteria during spray drying.

In this study we focused on drying of sessile single droplets to evaluate this hypothesis. The sessile single droplet drying method employs drying of small droplets, mimicking the spray drying process, and it allows us to visually monitor the particle morphology development and analyse bacterial survival for each droplet after drying. Different drying matrices were selected (trehalose, xylose, whey proteins, maltodextrins) that lead to distinct particle morphologies and have different physicochemical properties (e.g. different glass transition temperatures). Lactobacillus plantarum WCFS1 was used as a model probiotic bacterium. A systematic set of experiments was conducted to distinguish between the direct effect of the matrix on the survival of bacteria, and the effect of morphology, indirectly influenced via the rheological properties of the matrix (Siemons et al., 2020a). The results may contribute to the development of spray drying processes leading to higher bacterial survival.

\subsection{Materials and methods}

\subsubsection{Carrier materials}

Five types of drying matrices were investigated for their protective effects during drying of L. plantarum WCFS1 cells, i.e. whey protein, trehalose, maltodextrin dextrose equivalence 19 , maltodextrin dextrose equivalence 5 , and xylose. Whey proteins, purity $97.0-98.4 \%$ (BiPro ${ }^{\circ}$, Davisco, Switzerland), trehalose (Sigma Aldrich, USA), and xylose (Sigma Aldrich, USA) drying matrices were prepared at a dry matter concentration of $20 \%(\mathrm{w} / \mathrm{w})$. The maltodextrin matrices (Roquette, France) had a dry matter concentration of $20 \%(w / w)$, $30 \%(\mathrm{w} / \mathrm{w})$ or $40 \%(\mathrm{w} / \mathrm{w})$ before drying. The protein matrices were stirred overnight at $4^{\circ} \mathrm{C}$ to ensure complete hydration, while the carbohydrate matrices were stirred for a minimum of 30 minutes. All components were dissolved in demineralized water.

\subsubsection{Microorganisms and pre-culture}

Lactobacillus plantarum WCFS1 was obtained from the in-house strain collection. Preculture of this strain was performed according to the method described by Vaessen et al. (2018). L. plantarum WCFS1 was plated from a frozen stock on De Man, Rogosa and Sharpe (MRS) agar. The plates were incubated microaerobically at $30^{\circ} \mathrm{C}$ for $60-70$ hours. After incubation, the plates were stored at $4^{\circ} \mathrm{C}$ until further use. From these plates a single colony was transferred into $10 \mathrm{ml} \mathrm{MRS}$ broth and grown for $24 \pm 2$ hours at $30^{\circ} \mathrm{C}$. This culture was subsequently diluted 1:100 into fresh MRS broth and incubated overnight for $17 \pm 1$ hour 
at $30^{\circ} \mathrm{C}$. This overnight culture was centrifuged at $13,500 \times g$ for 10 minutes. The $\mathrm{pH}$ of the supernatant was measured to check the growth of the culture and was always $3.9 \pm 0.1$. The remaining pellet was washed once with a washing buffer (for composition see Vaessen et al. (2018)) and centrifuged again using the same settings. After centrifugation the supernatant was discarded and the pellet was suspended in the carrier matrix. This culture in drying medium consisted of 3-4 $10^{9} \mathrm{CFU} / \mathrm{ml}$.

\subsubsection{Single droplet drying}

The drying experiments were done using the single droplet drying equipment described earlier (Siemons et al., 2020). The droplets were dispensed by a PipeJet ${ }^{\circledR}$ NanoDispenser (BioFluidix, Germany) using 200-S PipeJet ${ }^{\circledR}$ Pipes (Biofluidix, Germany) on a flat hydrophobic membrane (Tetratex ${ }^{\oplus}$ ePTFE 3104 Polytetrafluoroethylene membrane, thickness $0.27 \mathrm{~mm}$ ) (Donaldson Nederland B.V., The Netherlands). Droplets were dried for 20 seconds in a heated air flow $(\mathrm{RH}=0 \%)$ at $90^{\circ} \mathrm{C}$, at a flow velocity of $0.3 \mathrm{~m} / \mathrm{s}$. Deposited droplets had an initial radius $\left(R_{0}\right)$ of $100 \pm 20 \mu \mathrm{m}$ and their size and shape were recorded with a camera. The obtained sequences were analysed for the initial droplet size and locking point via image analysis using Image J software (National Institute of Health, USA). The locking point, which represents the onset of solidity and the morphology development, was defined as the first visual observation of shape deviation of the drying droplet during video analysis. The experiments were carried out in duplicate at least.

After drying, the particles were studied using scanning electron microscopy (SEM). For SEM analysis, samples were fixed on a sample holder using carbon adhesive tabs. SEM images were taken at 5 kV, 3.74 pA, using a Phenom G2 Pure SEM (Thermo Fischer Scientific, The Netherlands). For all droplets, a full image and a topographic image were made using a high sensitivity backscatter electron detector (Thermo Fischer Scientific, The Netherlands). The SEM analyses were performed at least in duplicate.

For each experiment with bacteria, first a droplet was dispensed and subsequently the membrane with the droplet was immediately transferred into a vial containing $1 \mathrm{ml}$ PBS. This was done at least in duplicate and these droplets served as the control, without drying. Additionally for each experiment two to six droplets were dried for 20 seconds at $90^{\circ} \mathrm{C}$. After drying, these droplets were kept on the membrane for maximum 2 hours until subsequent survival analysis. For each dispensed droplet the exact droplet volume was determined by 
stroboscopic images analysed by the Biofluidix Control Software V2.9 (BioFluidix, Germany) and used for the calculation of the survival.

\subsubsection{Survival analysis}

The dried droplets were rehydrated by transferring the membrane with the dried droplet into vials containing $1 \mathrm{ml}$ PBS. After at least 10 minutes of rehydration, this culture was decimally diluted by pipetting $50 \mu \mathrm{l}$ of this rehydrated culture into $450 \mu \mathrm{l}$ PBS. The control droplets, which were dispensed but not dried, were also decimally diluted in the same way. Subsequently, the diluted cultures were plated in duplicate on MRS agar plates and incubated for $48-96$ hours at $30^{\circ} \mathrm{C}$ under microaerobic conditions. After incubation, the plates were counted and subsequently these counts were used to calculate the survival. Firstly, for each droplet the average CFU of the duplicate plates was used to calculate the $\mathrm{CFU} / \mathrm{nl}$ of the initial droplet by taking into account the dilutions and the exact volume of the dispensed droplet. Secondly, the survival was calculated by dividing the average CFU/nl of the dried droplet by the average $\mathrm{CFU} / \mathrm{nl}$ of the control droplets.

\subsubsection{Experimental setup}

All drying experiments with bacteria were carried out at least in duplicate, with each replicate being a fully independent experiment performed with another pre-culture and on another day. Additionally, for each experiment, multiple non-dried control and dried droplets were taken into account, to minimize the experimental errors. The morphology development during single droplet drying was also evaluated for at least three droplets.

\subsection{Results and discussion}

\subsubsection{Particle morphologies after single droplet drying}

Trehalose, whey protein and maltodextrin DE19 solutions were dried at $90^{\circ} \mathrm{C}$ using the sessile single droplet drying platform. The morphology development during drying was followed using a camera. In the initial phase of the drying process, the droplets of the suspensions decreased in size with similar rates, and remained spherical. After this initial drying phase, skin formation occurs due to an increased viscosity near the droplet surface. From the onset of the skin formation, often referred to as the locking point, the particle morphology starts to develop (Both et al., 2018; Siemons et al., 2020a). In droplets with 20\% $(w / w)$ whey proteins we observed that a vacuole started to develop shortly after the locking point (Fig. 6.1A). The final whey protein particle contained a large vacuole and had a smooth 
surface. The same morphology for whey protein particles was also observed in previous single droplet drying studies with droplets of a larger initial radius of $\sim 500 \mu \mathrm{m}$ at varying drying temperatures and whey protein concentrations (Both et al., 2018; Bouman et al., 2016; Sadek et al., 2013). Also Khem et al. (2016) observed this morphology after spray drying of Lactobacillus plantarum in the presence of whey proteins. The droplets with $20 \%$ $(w / w)$ trehalose shrank continuously until a small, dense and spherical particle was obtained (Fig. 6.1B). For trehalose no clear onset of skin formation could be observed. Droplets with 20\% (w/w) maltodextrin DE19 formed a dented morphology (Fig. 6.1C), which resembled the morphology of dried maltodextrin DE21 as was described before by Siemons et al. (2020a). In a spray drying study, Paramita et al. (2010) also found dented particles with high maltodextrin concentrations and smoother particles with high trehalose concentrations for mixtures of gum arabic and these components. Overall, the different final particle morphology types that we observed were a smooth dense particle, a smooth particle with a large vacuole and a dented particle.

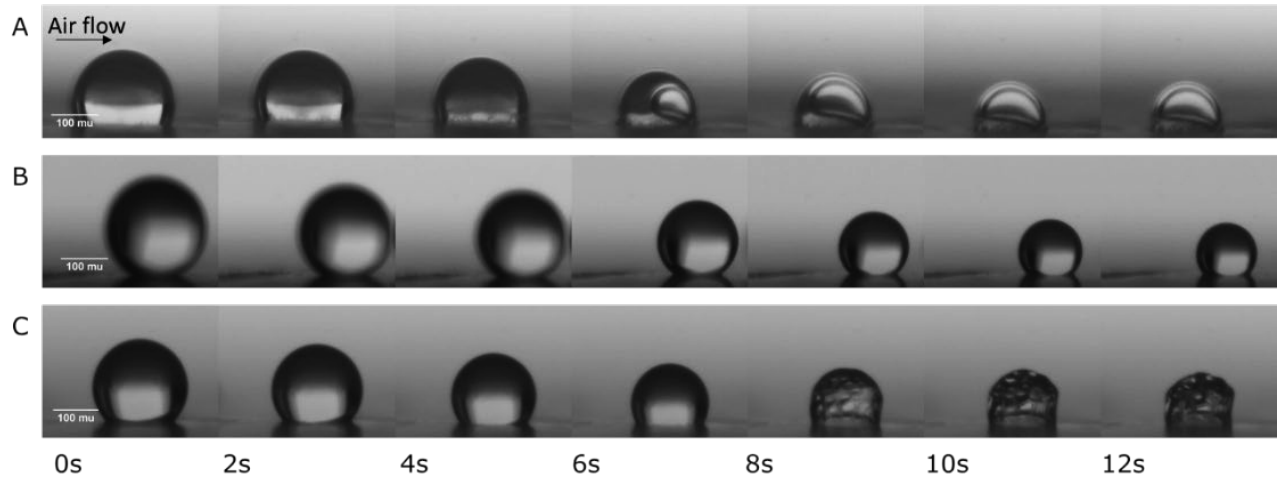

Figure 6.1 Particle morphology development during single droplet dying at $90^{\circ} \mathrm{C}$ of whey proteins (A), trehalose (B) and maltodextrin DE19 (C) at $20 \%(w / w)$ initial solids concentration. Air flow came from the left as indicated in the figure by the arrow.

To link the microbial survival after single droplet drying to the particle morphology, we selected components with different properties but leading to similar morphologies. For this purpose xylose, $20 \%(\mathrm{w} / \mathrm{w})$ maltodextrin DE5 and $30 \%(\mathrm{w} / \mathrm{w})$ maltodextrin DE5 were selected. Xylose is a monosaccharide that results in dense and smooth particles that are similar as those obtained with trehalose (Fig. 6.2). Drying of $20 \%(w / w)$ maltodextrin DE5 results in mostly dented particles, similar to maltodextrin DE19. However, maltodextrin 
DE19 had coarser edges and more smaller indentations, whereas maltodextrin DE5 had larger indentations with smoother edges (Fig. 6.2). Interestingly, when $30 \%(\mathrm{w} / \mathrm{w}$ ) maltodextrin DE5 was dried, a particle with a large vacuole was formed, similar to the morphology of whey protein. For almost all of the drying matrices, we observed consistent development of similar morphology for each dried particle; only in few cases for $20 \%(w / w)$ maltodextrin DE5 a vacuole was observed similar to our observations for drying of $30 \%$ (w/w) maltodextrin DE5 (Fig. 6.2). Therefore, both types of morphologies are displayed in figure 6.2. Additional time series for all components can be found in supplementary figure S.6.2.

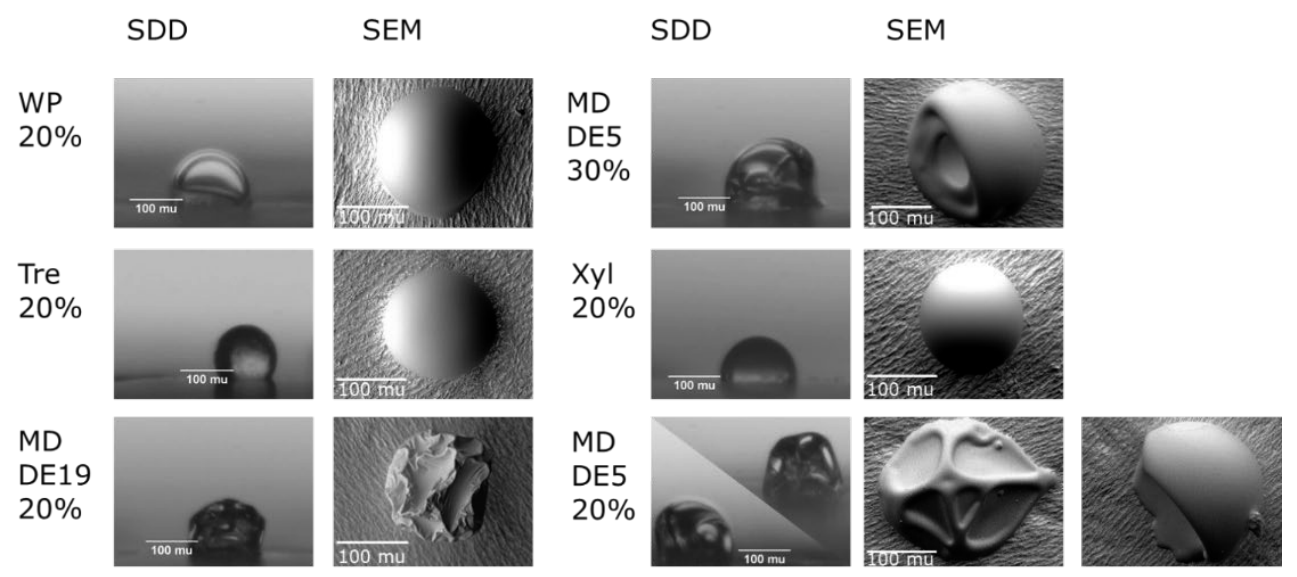

Figure 6.2 Final particle morphologies after single droplet drying at $90^{\circ} \mathrm{C}$ of various drying matrices. $W P=$ whey proteins, $T r e=$ trehalose, $M D D E 19=$ maltodextrin $D E 19, X y l=x y l o s e, M D$ $D E 5=$ maltodextrin DE5. The initial solids concentrations are indicated in percentages and scale bars are indicated.

\subsubsection{Survival of $L$. plantarum WCFS1}

Survival of Lactobacillus plantarum WCFS1 after single droplet drying was evaluated for all drying matrices described in the previous section (Fig. 6.2). The survival was comparable for drying matrices that yielded similar particle morphologies (Fig. 6.3). The highest survival was observed with the matrices that yield dense smooth particles, namely xylose $(90 \%)$ and trehalose (78\%). The drying matrices that led to a smooth and hollow morphology resulted in moderate survival of $51 \%$ for whey proteins and $35 \%$ for $30 \%(w / w)$ maltodextrin DE5. Survival was lowest in drying matrices that resulted in dented particles, namely $2 \%$ for maltodextrin DE19 and $8 \%$ for 20\% (w/w) maltodextrin DE5. 


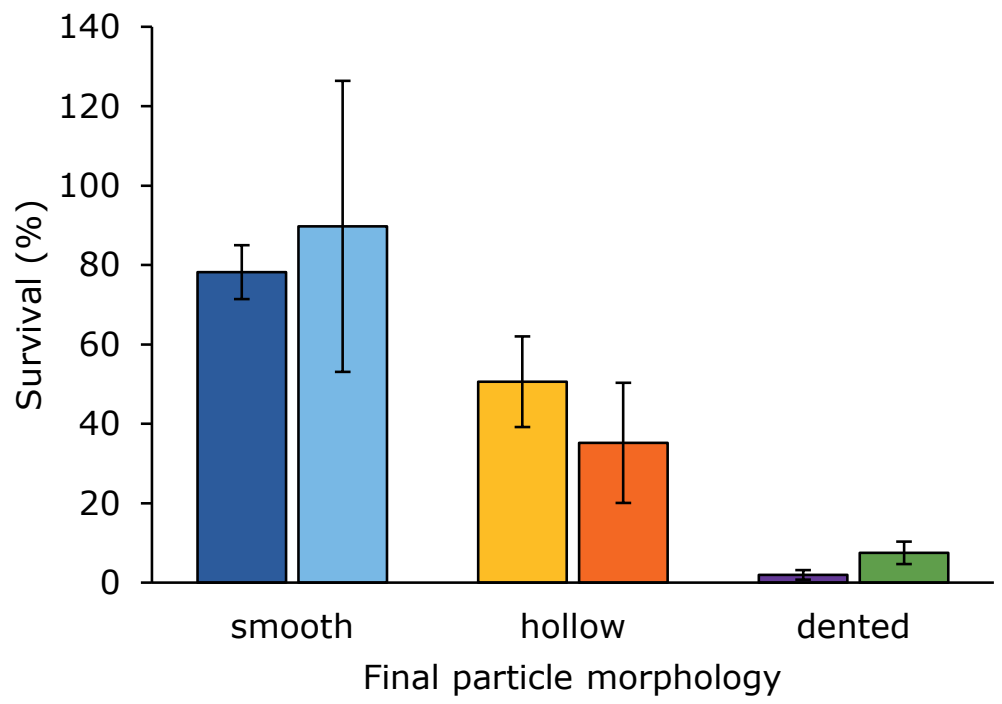

Figure 6.3 Survival of L. plantarum WCFS1 after single droplet drying at $90^{\circ} \mathrm{C}$ in various drying matrices. The drying matrices were (from left to right): $20 \%(w / w)$ trehalose (dark blue), 20\% (w/w) xylose (light blue), 20\% (w/w) whey proteins (yellow), 30\% (w/w) maltodextrin DE5 (orange), 20\% (w/w) maltodextrin DE19 (purple) and 20\% (w/w) maltodextrin DE5 (green). Error bars represent standard deviations of independent replicates $(n \geq 2)$.

This lower survival for maltodextrin is in agreement with the study of Broeckx et al. (2017), who evaluated the survival of $L$. rhamnosus GG after spray drying, and found a higher survival with trehalose and lactose compared to maltodextrin DE6. Other studies reported a similar effect when comparing survival of L. plantarum WCFS1 in trehalose and maltodextrin (DE 19, DE 16 and 6) after spray drying (Perdana et al., 2014b; Vaessen et al., 2020). On the other hand, Vaessen et al. (2020) found a similar survival of L. plantarum WCFS1 in whey protein and trehalose after spray drying and Khem et al. (2015)found a higher survival of Lactobacillus plantarum A17 after single droplet drying in whey proteins compared to trehalose as carrier matrix. This latter study ascribes this to the high temperature increase rates observed for trehalose droplets, while the temperature increase for whey protein droplets was more gradual. This is not in line with our observations. We speculate that the difference in drying conditions and drying time, e.g. $240 \mathrm{~s}$ in their study vs $20 \mathrm{~s}$ in our study, is relevant here. 
The observed trends regarding survival of L. plantarum WCFS1 in the various drying matrices suggest that there is a relation between particle morphology development and survival of bacteria in this carrier matrix. Though, before drawing further conclusions based on these observations, several aspects require further investigation. First of all, maltodextrin DE5 was used with two different concentrations; with a solids content of $20 \%(w / w)$ mainly a dented particle was formed, and with a solids content of $30 \%(w / w)$ a smooth particle with a large vacuole was formed. In line with the correlation that we found, the survival with $20 \%$ DE5 was low, while the survival with $30 \%$ was moderate. This means that the same type of carrier matrix with different morphology gives different survival.

\subsubsection{Effect of solids content and duration of constant rate period}

We evaluated the survival of L. plantarum WCFS1 in maltodextrin DE19 and maltodextrin DE5 as drying matrices at various solids contents. Survival in maltodextrin DE5 increased from $8 \%$ to $35 \%$ with increasing solids content from $20 \%(w / w)$ to $30 \%(w / w)$ and changing morphology from predominantly dented to hollow (Fig. 6.2). For maltodextrin DE19 the survival only increased from $2 \%$ to $5 \%$ with the same increase in solids content (Fig. 6.4A). Further increasing the solids content to $40 \%(\mathrm{w} / \mathrm{w})$ resulted in a slightly higher survival of $13 \%$. For all of these matrices we found dented particle morphologies (Fig. 6.4B), where only the edges and indentations of the particles became less coarse upon increasing the initial solids content.

In literature, different effects of solids content have been reported for survival of bacteria after drying. For example, Perdana et al. (2014b) found that $2 \%(w / w)$ trehalose was sufficient to protect $L$. plantarum WCFS1 during drying, and found no further increase in survival by increasing the trehalose content further. Another study reported that increasing the content of gelatin, gum arabic or soluble starch from 10 to 20 and $30 \%(w / w)$ resulted in a lower survival of Bifidobacteria compared to $10 \%$ (w/w) (Lian et al., 2002). On the other hand, Ghandi et al. (2012b) reported an increase in survival of Lacotococcus lactis when this strain was dried at higher solids contents. For example, the survival increased from 9 to $22 \%$ when increasing the solids content of a lactose-sodium caseinate carrier from 10 to $35 \%$ $(w / w)$. Wurth et al. (2018) described a decrease in survival of $L$. paracasei when increasing the solids content of a skim milk concentrate from $12.5 \%(w / w)$ to $35 \%(w / w)$. Additionally, they found a strong correlation between the particle size of the spray dried particle and 
bacterial survival; a smaller particle resulted in better survival. They relate this to the solids content, with lower solids contents leading to a smaller final particle and a prolonged constant rate period (Würth et al., 2018). To evaluate whether the duration of this constant rate drying period can be linked to survival, we used the locking point as measure for the duration of the constant rate drying period (Fig. 6.5).
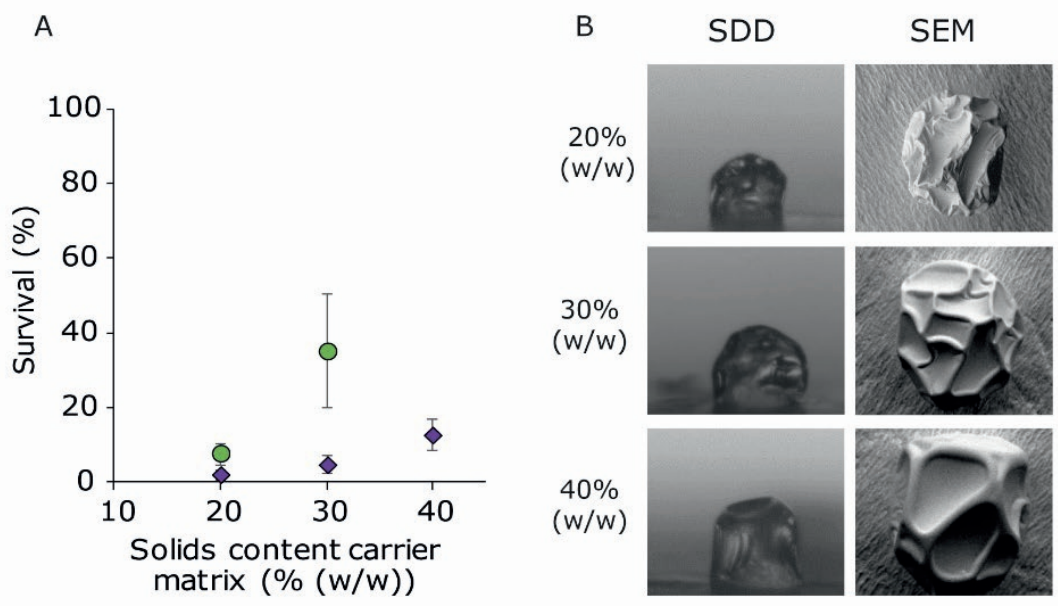

Figure 6.4 Effect of solids content on survival of L. plantarum WCFS1 (A) and particle morphology (B). (A) Survival of L. plantarum WCFS1 in maltodextrin DE19 (purple diamonds) and maltodextrin DE5 (green circles). Error bars indicate standard deviations of independent replicates (B) Final particle morphologies after single droplet drying at $90^{\circ} \mathrm{C}$ of maltodextrin DE19 at various solids content, i.e. 20,30 and $40 \%(w / w)$.

The determination of the locking point was based on the visual analysis of droplet drying movies. At the locking point, the spherical shape of the droplet becomes distorted and shows first signs of cavity formation or denting (Siemons et al., 2020a). The droplets with trehalose and xylose did not exhibit a clear locking point. These droplets have a longer constant rate period (Supplementary Fig. S.6.3), and did not show any distortions from spherical shape, eventually resulting in small spherical particles. This was expected as for these small sugars the solute diffusivity is faster or comparable to the drying rate (Vehring, 2008; Vehring et al., 2007). Therefore, the saturation at the droplet surface is reached late in the drying process with relatively homogeneous distribution of these sugars in the droplet. Overall, we did not observe a consistent relation between the locking point time, 
i.e. the duration of the constant rate period and the survival of L. plantarum WCFS1 after drying (Fig. 6.5).

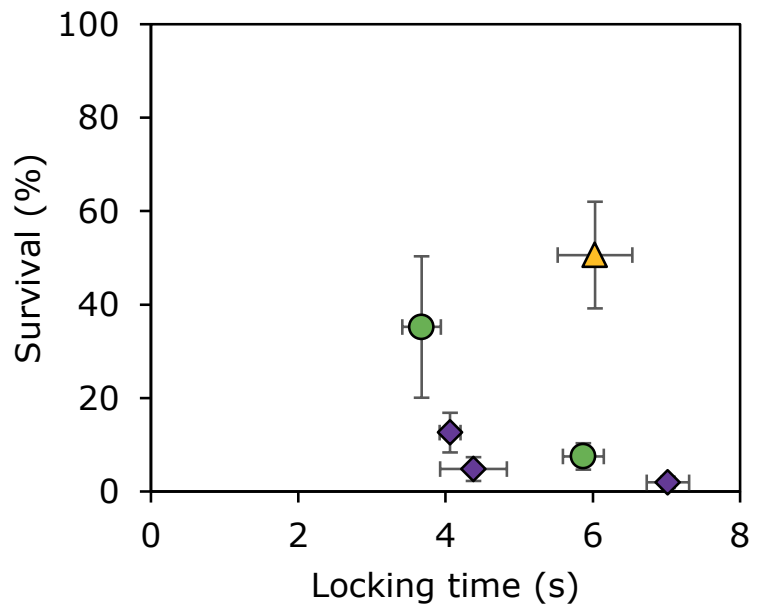

Figure 6.5 Survival of L. plantarum WCFS1 after single droplet drying in several drying matrices as a function of the locking time. The drying matrices are whey proteins (yellow triangles), maltodextrin DE5 (green circles) and maltodextrin DE19 (purple diamonds). Horizontal error bars represent the standard error of the locking point analysis and vertical error bars represent the standard deviation of bacterial survival. The matrices with trehalose and xylose did not exhibit a clear locking point.

Several single droplet drying studies described that the inactivation during the constant rate period is limited, probably due to the low (wet bulb) temperature during this period, instead several orders of magnitude of inactivation took place in the second, falling rate period of drying (Khem et al., 2015; Zheng et al., 2015). In this period, diffusion limitation is dominant and the droplet temperature increases gradually towards the air temperature. If this happens when the droplet is still high in water internally, this will result in strong thermal inactivation. Therefore, it is expected that the microbial survival rate is more strongly coupled to the processes in the falling rate period, than this is in the constant rate period. 


\subsubsection{Effect of physicochemical matrix properties}

The physical state of the matrix during drying, i.e. glassy or non-glassy, is considered important to the survival of probiotics during and after drying (Santivarangkna et al., 2011). As the evaporation of water progresses during drying, the viscosity of the droplet surface will increase until the surface reaches a critical temperature and moisture content, at which the matrix will behave as a glass. The extremely high viscosity of amorphous glassy matrices ( $\geq 10^{12}$ Pa.s) effectively halts diffusion-controlled deterioration reactions, improving the chemical and physical stability of the embedded bacterial cells (Aschenbrenner et al., 2012; Schutyser et al., 2012).

Carrier materials with a high glass transition temperature $\left(T_{g}\right)$ are expected to provide a glassy matrix earlier during droplet drying or soon after drying upon cooling (Perdana et al., $2014 \mathrm{~b}, 2012)$. We estimated the $\mathrm{T}_{\mathrm{g}}$ as function of moisture content for every component studied in this work using the Couchman-Karasz theory (Fig. 6.6A). This theory is further explained in supplementary information S.6.1.
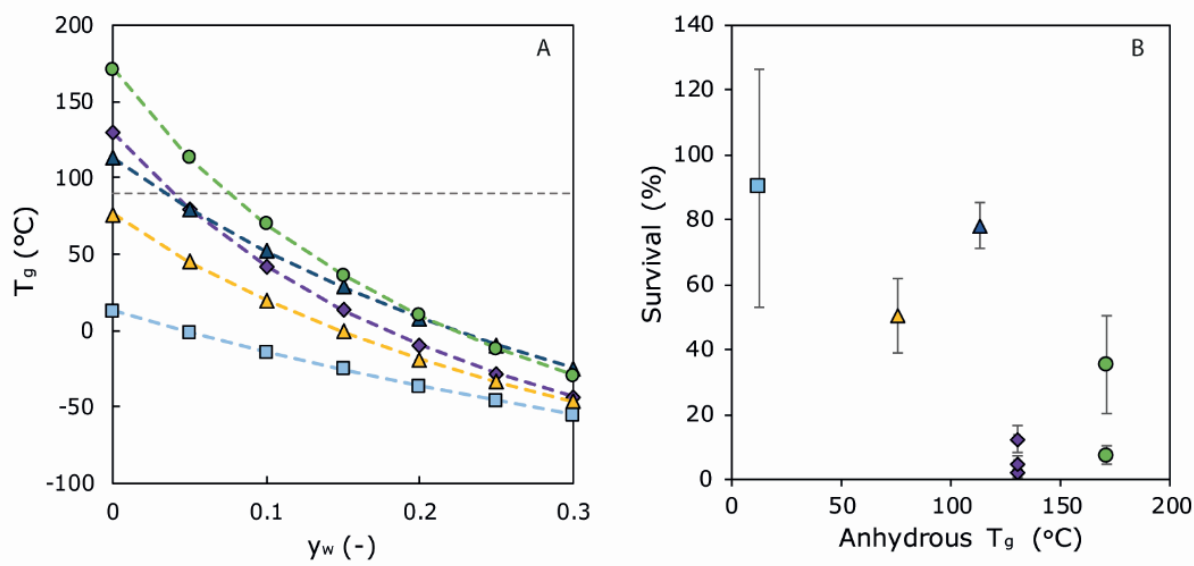

Figure 6.6 (A) Glass transition temperatures as function of moisture content $\left(y_{w}\right)$ as calculated with Couchman-Karasz. The drying air temperature of $90^{\circ} \mathrm{C}$ is indicated with a dotted line. (B) Survival of L. plantarum WCFS1 after single droplet drying as function of anhydrous glass transition temperature. The components are trehalose (blue triangles), xylose (light blue squares), whey proteins (yellow triangles), maltodextrin DE5 20 and 30\% $(w / w)$ (green circles) and maltodextrin DE19 20, 30 and 40\% (w/w) (purple diamonds). 
Maltodextrin DE5 has the highest $\mathrm{T}_{\mathrm{g}}$ for moisture contents below $0.3 \mathrm{~kg}$ water $/ \mathrm{kg}$, followed by trehalose, maltodextrin DE19, whey protein and xylose (Fig. 6.6A). In Fig. 6.6B the survival is given as function of the $T_{g}$ of the anhydrous matrix. Fig. $6.6 \mathrm{~B}$ indicates that a higher $\mathrm{T}_{\mathrm{g}}$ of a matrix is not directly correlated to higher retention of viability after drying. In particular, the survival percentages found for L. plantarum WCFS1 after drying in maltodextrin and xylose matrices contradict the hypothesis that matrices with high $\mathrm{T}_{\mathrm{g}}$ offer higher degree of survival after drying.

While entering the glassy state at an early stage during drying in principle should offer better survival, it also halts the drying process itself by reducing the water diffusivity (Ribeiro et al., 2002; Zobrist et al., 2011). For maltodextrin matrices, it is expected that the skin that is formed upon concentration will quickly move towards the glass transition, where the viscosity increases dramatically, and water diffusivity is strongly reduced. The decreased water diffusivity causes the droplet temperature to increase, while the viscosity of the skin remains high due to ongoing evaporation. The relatively fast skin formation followed by a quick approach of the glassy state may result in a short constant rate period, and a quickly rising droplet temperature, while the droplet interior may still have relatively high water content. Especially the combination of elevated temperature and high moisture content may lead to extensive microbial inactivation.

Whey proteins undergo a colloidal glass transition already above a solids concentration of $50 \%$ (w/w) (Both, Siemons, Boom, \& Schutyser, 2019a; Both, Tersteeg, Boom, \& Schutyser, 2019b). It is expected that this transition is based on jamming, leading to elasticity, and not just an increase in viscosity. Therefore, as soon as the skin is formed, it will withstand the surface stresses and retains its shape, while at the same time lower internal pressures develop due to the ongoing evaporation (Both et al., 2018; Bouman et al., 2016). At some point, a weak point in the skin will yield, and a cavity is formed. In this case, the glassy colloidal skin does not directly result in quickly rising droplet temperatures, since the cavity serves as an evaporation vent (Bouman et al., 2016). Hence, bacteria may be better protected against heat and high moisture content due to prolonged constant drying via the cavity. It may even be that the vacuole formation causes a longer constant rate period than what was predicted based on locking point analysis. The latter might also explain higher survival for maltodextrin DE5 at $30 \%(w / w)$. 
Trehalose is a disaccharide, while xylose is a monosaccharide. These molecules have relative high diffusivities compared to oligo- or polysaccharides (van der Sman and Meinders, 2013). Therefore, these components will accumulate more slowly below the surface, and the droplet will lose more of its moisture in the constant rate regime, where the temperature is low and microbial inactivation is therefore limited. Indeed we observed via image analysis that the constant rate regime continued for a longer time with these matrices (Fig. S.6.3). These droplets only come into the falling rate period at a later stage, when the concentration of water has reduced strongly. The latter implies that the period of microbial inactivation has shortened, and the lower water content at the start of the falling rate period may additionally slow down the inactivation.

On the cellular level, the protective effect of low molecular weight carbohydrates such as trehalose and xylose is often explained by their ability to depress the phase transition of the bacterial cell membrane due to interactions with the phospholipids (Bryant et al., 2001; Huang et al., 2017). Indeed, the low molecular weight components in our experiments provided the best protection during survival (Fig. 6.7). However, at higher molecular weights no relationship is observed between molecular weight and bacterial survival in this matrix. This differs from Perdana et al. (2014b), who observed a decreasing residual viability with increasing molecular weight if the molecular weight was higher than $2 \cdot 10^{3} \mathrm{~g} / \mathrm{mol}$. A complication in this comparison of survival and molecular weight is that maltodextrins are mixtures of smaller and larger carbohydrates, especially maltodextrin DE19 contains a considerable amount of low molecular weight components (Avaltroni et al., 2004). In addition to the importance of low molecular weight, the combination of low molecular weight and glass transition is also often described as an important aspect for the stabilization of bacterial cell membranes. Glass transition close to the membrane phospholipids might reduce the compressive stresses that force the membrane lipids from a fluid phase into a gel phase causing loss of membrane integrity (Bryant et al., 2001). For this effect components should be small enough to enter the spacing between membranes and have a high $T_{\mathrm{g}}$. Actually, this is not in agreement with the high survival of xylose, which has a very low glass transition temperature (Fig. 6.6). Despite the possible capability of xylose to enter the intermembrane spacing due to its low molecular weight, it is still likely that xylose does not vitrify during drying as the $T_{\mathrm{g}}$ values are far below the drying air temperature. This might also affect survival during storage after drying, which is of great 
importance for dried bacterial ingredients and requires further investigation since it has not been considered in our study.

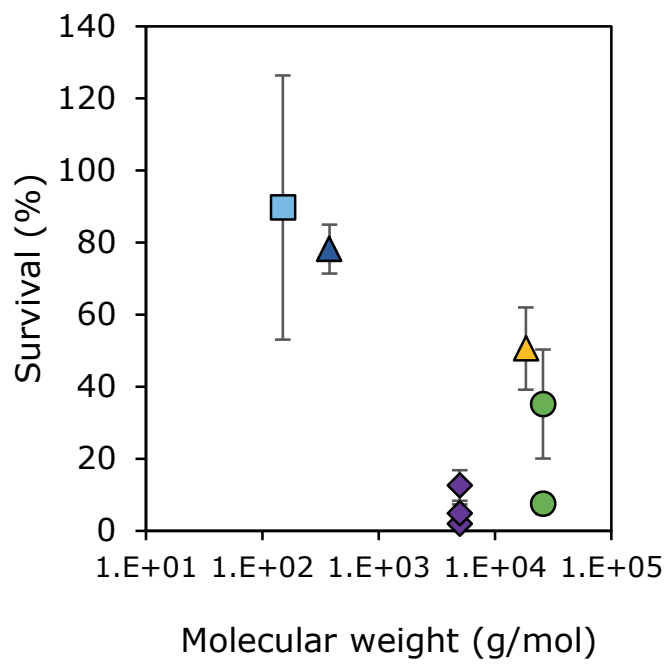

Figure 6.7 Survival of L. plantarum WCFS1 after single droplet drying as function of molecular weight of the component. The components are trehalose (blue triangles), xylose (light blue squares), whey proteins (yellow triangles), maltodextrin DE5 20 and 30\% (w/w) (green circles) and maltodextrin DE19 20, 30 and 40\% (w/w) (purple diamonds). Vertical error bars represent the standard deviation of bacterial survival.

We demonstrate that the clear relation that we found between the morphology and the microbial survival, is likely rooted in a complex interplay between matrix properties (glass transition) and drying dynamics (diffusion rates). However they are both the consequence of the same process; hence the strong correlation between these two resultants. This implies that the investigation of the morphologies may well offer a route towards better microbial survival during spray drying.

\subsection{Conclusions and outlook}

Drying of droplets having different matrices resulted in smooth, hollow or dented particles. Maltodextrin DE19 and 20\% (w/w) maltodextrin DE5 resulted in dented or folded particles; whey protein and $30 \%(\mathrm{w} / \mathrm{w})$ maltodextrin DE5 resulted in hollow particles, while trehalose and xylose resulted in smoothly spherical, dense particles. The survival of Lactobacillus plantarum WCFS1 that was embedded in these matrices showed clear correlation with 
these morphologies, with the smooth particles giving high survival, dented particles low survival, and hollow particles in between.

This strong correlation can be explained by a combination of drying dynamics and the glass transition, with high-molecular weight components like maltodextrins giving fast formation of a viscous shell, which folds into a dented geometry, allowing significant inactivation due to the long falling rate period at elevated temperatures. Small components such as xylose and trehalose exhibit a much longer constant rate period, and significant protection against inactivation during the shorter falling rate period. The hollow particles are the result of an elastic shell that resists folding, but offer a prolonged constant evaporation thanks to cavity formation allowing fast evaporation and therefore a longer low temperature period than the high-molecular weight maltodextrins.

Our results suggest that the morphology development is strongly correlated to the microbial survival, because it is fundamentally related to the same underlying mechanisms. Therefore it is an excellent phenomenon to use for optimizing microbial survival during spray drying.

Other bacterial strains and different drying matrices, including mixes of carbohydrates and proteins, should be tested to further support our findings. Single sessile droplet drying experiments offer the opportunity to probe many bacterial drying formulations and drying conditions to find promising particle morphologies leading to higher bacterial survival. 


\section{Supplementary information}

\section{S.6.1.Glass transition temperatures and molecular weights}

In our study, the glass transition temperature $T_{\mathrm{g}}$ of the different components was estimated as a function of moisture content. The relation was described using the theory of Couchman and Karasz (1978):

$$
T_{g}=\frac{y_{w} \Delta c_{P, w} T_{g, w}+y_{s} \Delta c_{p, s} T_{g, s}}{y_{w} \Delta c_{p, w}+y_{s} \Delta c_{p, s}}
$$

Here $y_{i}$ is the mass fraction ( $i=w, s$ for water and solute), $T_{g, i}$ is the glass transition temperature for the pure component $\left(T_{g, w}=139 \mathrm{~K}\right), \Delta c_{p, i}$ is the change in the specific heat at the glass transition. For $\Delta c_{p, w}$ a value of $1.91 \mathrm{~kJ} / \mathrm{kg} \cdot \mathrm{K}$ was used. Glass transition temperatures of maltodextrins were determined as described by Siemons et al. (2020a). The anhydrous glass transition temperature and the heat capacities of the solutes and the molecular weights of the components are provided in Table S.6.1.

Table S.6.1 Overview of materials and their anhydrous glass transition temperature, specific heat capacity and molecular weight.

\begin{tabular}{lllll} 
Pure components & $\mathbf{T}_{\mathbf{g}}\left({ }^{\circ} \mathbf{C}\right)$ & $\mathbf{\Delta} \mathbf{c}_{\mathbf{p}}\left(\mathbf{J} \cdot \mathbf{g}^{-\mathbf{1}} \mathbf{K}^{-\mathbf{1}}\right)$ & $\mathbf{M w}\left(\mathbf{g} \cdot \mathbf{m o l}^{-1}\right)$ & Reference \\
\hline Trehalose & 113 & 0.65 & 342 & (Kawai et al., 2005) \\
\hline Xylose & 13 & 0.95 & 150 & $\begin{array}{l}\text { (Kalichevsky et al., } \\
1993)\end{array}$ \\
\hline Whey proteins & 76 & 0.58 & 18200 & $\begin{array}{l}\text { (Nicolai et al., 2011; } \\
\text { Yang et al., 2010) }\end{array}$ \\
\hline Maltodextrin DE19 & 130 & 0.43 & 4978 & $\begin{array}{l}\text { (Castro et al., 2016; } \\
\text { Van Der Sman \& } \\
\text { Meinders, 2011) }\end{array}$ \\
\hline Maltodextrin DE5 & 171 & 0.43 & & $\begin{array}{l}\text { (Castro et al., 2016; } \\
\text { Van Der Sman \& } \\
\text { Meinders, 2011) }\end{array}$ \\
\hline
\end{tabular}




\section{S.6.2 Single droplet drying time series}

A

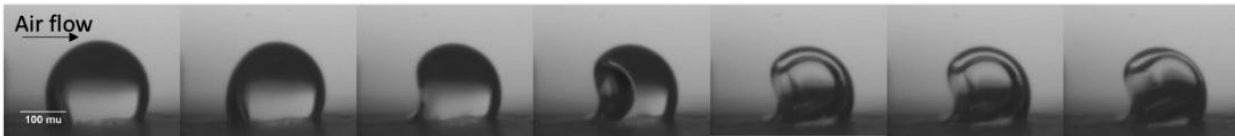

B

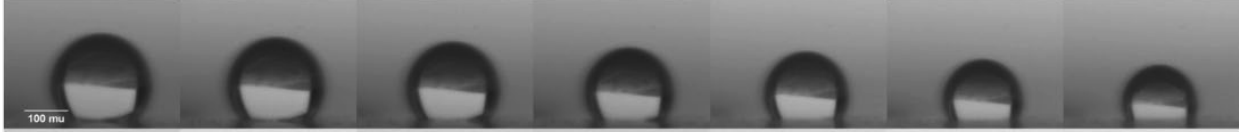

C

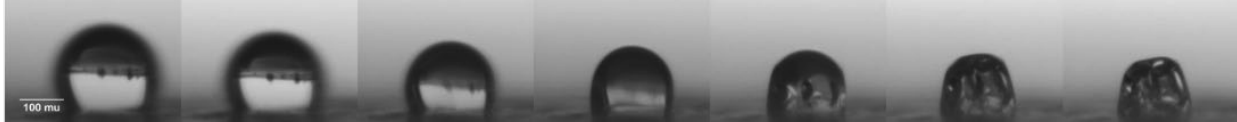

D

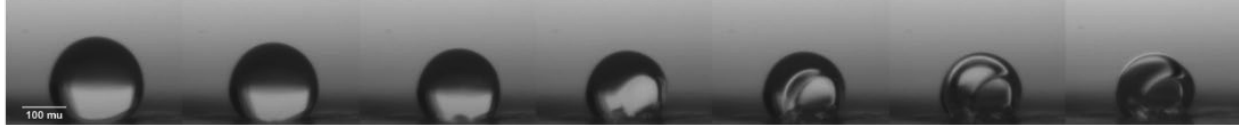

$\mathrm{E}$

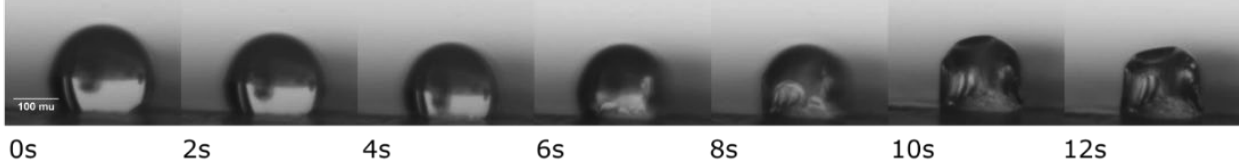

Figure S.6.2 Particle morphology development during single droplet dying at $90^{\circ} \mathrm{C}$ of maltodextrin DE5 30\% (w/w) (A), xylose 20\% (w/w) (B), maltodextrin DE5 20\% (w/w) (C), maltodextrin DE5 20\% (w/w) (D), maltodextrin DE19 40\% (w/w) (E). Air flow came from the left as indicated in the figure by the arrow. 


\section{S.6.3. Trehalose and xylose drying}

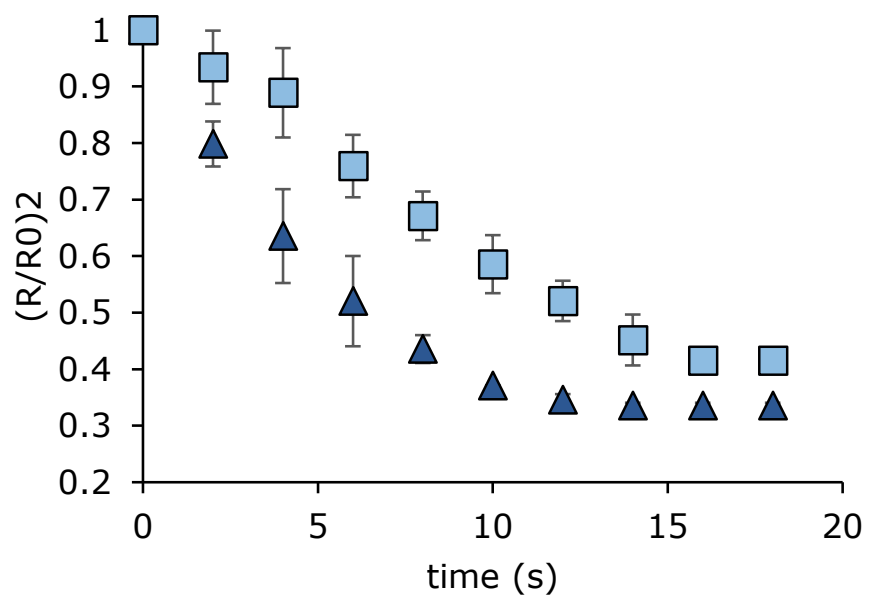

Figure S.6.3 Normalized squared radius as function of drying time for xylose (light blue squares) and trehalose (blue triangles). A linear decrease indicates a constant drying rate as described by Bouman (2016). The data were obtained from videos via image analysis. 
General discussion 


\subsection{Introduction}

Probiotics and starter cultures can be dried into a powder to extend their shelf life. For the application as a probiotic or starter culture it is essential that these bacteria retain their viability during drying and subsequent storage. However, inactivation of bacteria occurs during this process due to thermal and dehydration stresses. Therefore the aim of this thesis was to develop a pulsed electric field pre-treatment aiming at introducing protective solutes into the bacterial cells, to improve bacterial survival during drying and subsequent storage of the powders. This chapter discusses the main findings that were described in the previous chapters. In addition, other factors that can affect the efficacy of the PEF pre-treatment are discussed, as well as the correlation between intracellular trehalose and other compatible solutes and cell robustness. Finally, the potential implementation of a PEF pre-treatment in an industrial drying process of bacteria is discussed.

\subsection{Main Findings}

The first aim of this thesis was to develop a pulsed electric field treatment that results in increased intracellular protective solute concentrations, while maintaining a high viability. In chapter 2 we show that a PEF treatment with two pulses of $100 \mu$ s at 7.5, 10 or 12.5 $\mathrm{kV} / \mathrm{cm}$ led to increased intracellular trehalose concentrations in Lactobacillus plantarum WCFS1. Survival of this strain decreased when increasing the electric field strength. Therefore, based on the parameters tested in this chapter, the PEF treatment at $7.5 \mathrm{kV} / \mathrm{cm}$ resulted in an optimum balance between increased intracellular trehalose concentrations and relatively high survival of the PEF treatment. For the purpose of loading cells with protective solutes, a PEF treatment resulting in reversible membrane permeabilization is required. To investigate the reversibility of the induced membrane permeabilization, we applied fluorescent staining techniques in chapter $\mathbf{2}$ and 3. By staining the cells with the membrane impermeable propidium iodide (PI) before and after PEF, we showed that only approximately $10 \%$ of the bacterial population had a reversible permeabilized membrane. Resealing of the membrane occurred fast after PEF treatment, already within several seconds at maximum. In addition to the staining technique with PI before and after PEF treatment, a second staining technique was introduced in chapter 3 , in which we used two membrane impermeable stains, i.e. PI and SYTOX Green. Using this method larger reversible permeabilized fractions were observed compared to the first staining technique for several PEF conditions. This difference shows that despite that these techniques are fast and 
relatively easy methods to detect reversibility of membrane permeabilization, the choice of the method can affect the quantification of reversibility of membrane permeabilization. A critical electric field strength for membrane permeabilization in L. plantarum WCFS1 was determined around $5 \mathrm{kV} / \mathrm{cm}$.

The next step was combining the PEF pre-treatment with subsequent drying of $L$. plantarum WCFS1. To have a larger PEF volume compared to the $400 \mu$ l that was used in chapter 2 and 3, a larger batch PEF treatment chamber with a volume of $\sim 13 \mathrm{ml}$ was developed for the PEF experiments prior to drying in chapter $\mathbf{4}$ and $\mathbf{5}$. In this system a PEF treatment with 10 pulses of $100 \mu \mathrm{s}$ at $6.3 \mathrm{kV} / \mathrm{cm}$ was applied resulting in increased intracellular trehalose or lactose concentrations while maintaining high culture survival. Subsequent freeze drying and storage of PEF pre-treated cultures of L. plantarum WCFS1 did not result in higher survival compared to the control cultures without PEF pre-treatment (chapter 4). In these experiments both the PEF treatment and subsequent freeze drying were done in the same medium, containing either trehalose or lactose as protective carbohydrate. Additional analysis of intracellular trehalose and lactose concentrations throughout the drying process revealed that intracellular trehalose concentrations in control cultures without PEF pretreatment greatly increased upon freezing before freeze drying. For drying in a lactose medium, an increase in intracellular lactose was only observed after freeze drying, not after freezing. This increase in intracellular trehalose and lactose of the cultures without PEF pretreatment could explain why no difference in survival was observed between the PEF pretreated and control cultures. Also after spray drying and reconstitution of the powder, trehalose and/or lactose were found intracellularly in L.plantarum WCFS1 when the culture was spray dried in these carbohydrates. Based on these findings we continued in chapter $\mathbf{5}$ with evaluating the added value of a PEF pre-treatment in trehalose upon spray drying in other drying media. Interestingly, PEF pre-treatment in trehalose resulted in a doubled survival of Lactobacillus plantarum WCFS1 after spray drying in reconstituted skim milk. Also during storage, survival of PEF pre-treated cultures remained higher in this carrier matrix compared to cultures without PEF. In other drying matrices, namely trehalose, whey protein and maltodextrin, the PEF pre-treatment in trehalose had (almost) no beneficial effect on survival after drying and storage. Remarkably, PEF pre-treatment in lactose resulted also in a large increase in survival of L. plantarum WCFS1 after spray drying in RSM. Though, the better survival after spray drying in RSM upon PEF pre-treatment was not found for another lactic acid bacterium, namely L. lactis FM03. These findings show that the 
applicability of the PEF pre-treatment to enhance robustness depends on the carrier matrix during drying, as well as on the bacterial strain.

In chapter $\mathbf{5}$ we also observed that different carrier matrices lead to differences in survival during spray drying of cells that were not PEF pre-treated. Why some matrices result in better protection compared to others remains unclear. Therefore, we studied the influence of the carrier matrix on survival of $L$. plantarum WCSF1 during sessile single droplet drying in chapter 6. More specifically, we focused on the question whether particle morphology, as influenced by the properties of the carrier matrix and the drying conditions, could be related to survival after drying. Indeed, a correlation was found between final particle morphology and survival of $L$. plantarum WCFS1. Survival in smoothly spherical and dense particles was the highest, followed by the smooth and hollow particles and survival was lowest in dented particles. The relation between survival rates and particle morphology could not be explained by a single matrix property only, but by a combination of glass transition and drying dynamics in the drying droplets.

\subsection{PEF pre-treatment to increase intracellular trehalose concentrations}

Many factors can influence the outcome of PEF treatment, such as processing parameters, microbial factors and treatment medium characteristics (Table 1.1). Most of these parameters have been investigated for their effect on bacterial survival after PEF treatment in the context of bacterial inactivation (Álvarez et al., 2003; Saldaña et al., 2009; Timmermans et al., 2014; Wouters et al., 2001a). For the purpose of loading bacterial cells with high concentrations of carbohydrates not only survival of the PEF treatment is essential, but also the final intracellular carbohydrate concentrations. This balance between survival and intracellular concentrations needs to be taken into account when designing a PEF process aiming at increasing intracellular carbohydrate concentrations. In the following sections we will discuss the effect of temperature, membrane fluidity and strain variability on the survival and intracellular trehalose content after PEF treatment. All PEF treatments in these sections were carried out with the batch PEF system described in detail in Vaessen et al. (2019).

\subsubsection{PEF and membrane fluidity}

In addition to the effect of the pulse parameters electric field strength, number of pulses and pulse duration (chapter 2 and 3), we also investigated the effect of temperature and 
the treatment medium. Temperature plays an important role in the inactivation effect of PEF treatment (Cebrián et al., 2016). In the batch scale set-up described in chapter 4 we evaluated the use of different PEF temperatures on the uptake of intracellular trehalose and survival of $L$. plantarum WCFS1 (Fig. 7.1). A PEF temperature of $35^{\circ} \mathrm{C}$ resulted in lower survival and much lower intracellular trehalose compared to PEF treatments at 21 and $10^{\circ} \mathrm{C}$. Overall, a temperature of $21^{\circ} \mathrm{C}$ resulted in the highest intracellular trehalose concentrations, especially when applying $10-12$ pulses of $6.3 \mathrm{kV} / \mathrm{cm}$ and a pulse duration of $100 \mu \mathrm{s}$. The decrease in survival after PEF treatment when increasing the PEF temperature is in line with the effects of temperature that are observed in PEF inactivation studies (Aronsson and Rönner, 2001; Cebrián et al., 2016; Timmermans et al., 2014).

What might play a role in this temperature effect is the fluidity of the membrane. The cellular membrane is damaged by the PEF treatment, as PEF treatment can lead to pore formation and permeabilization of the cellular membrane. Membrane fluidity can be defined as the relative diffusional motion of lipids within cell membranes and affects cell permeability and membrane functionality (Mykytczuk et al., 2007). Higher temperatures result in an increased membrane fluidity, as was determined by measuring the fluorescence anisotropy of the fluorescent probe 1,6-diphenyl-1,3,5-hexatriene (DPH) (Fig. 7.1.C). When $\mathrm{DPH}$ is embedded in the cellular membrane, the mobility of the molecule is limited by the phospholipid membrane structure. The fluidity of the phospholipid membrane determines the degree of mobility of DPH, which can be measured by fluorescence anisotropy. This fluorescence anisotropy is inversely related to the membrane fluidity (Lentz, 1989). Although the DPH measurements resulted in relatively high standard deviations of the biologically independent replicates, the slope of the curve was significant $(P<0.05)$, thus indeed indicating that at a higher temperature the membrane fluidity of L. plantarum WCFS1 increased. Similar fluorescence anisotropy results were found for L. bulgaricus strains by Meneghel et al. (2017). 

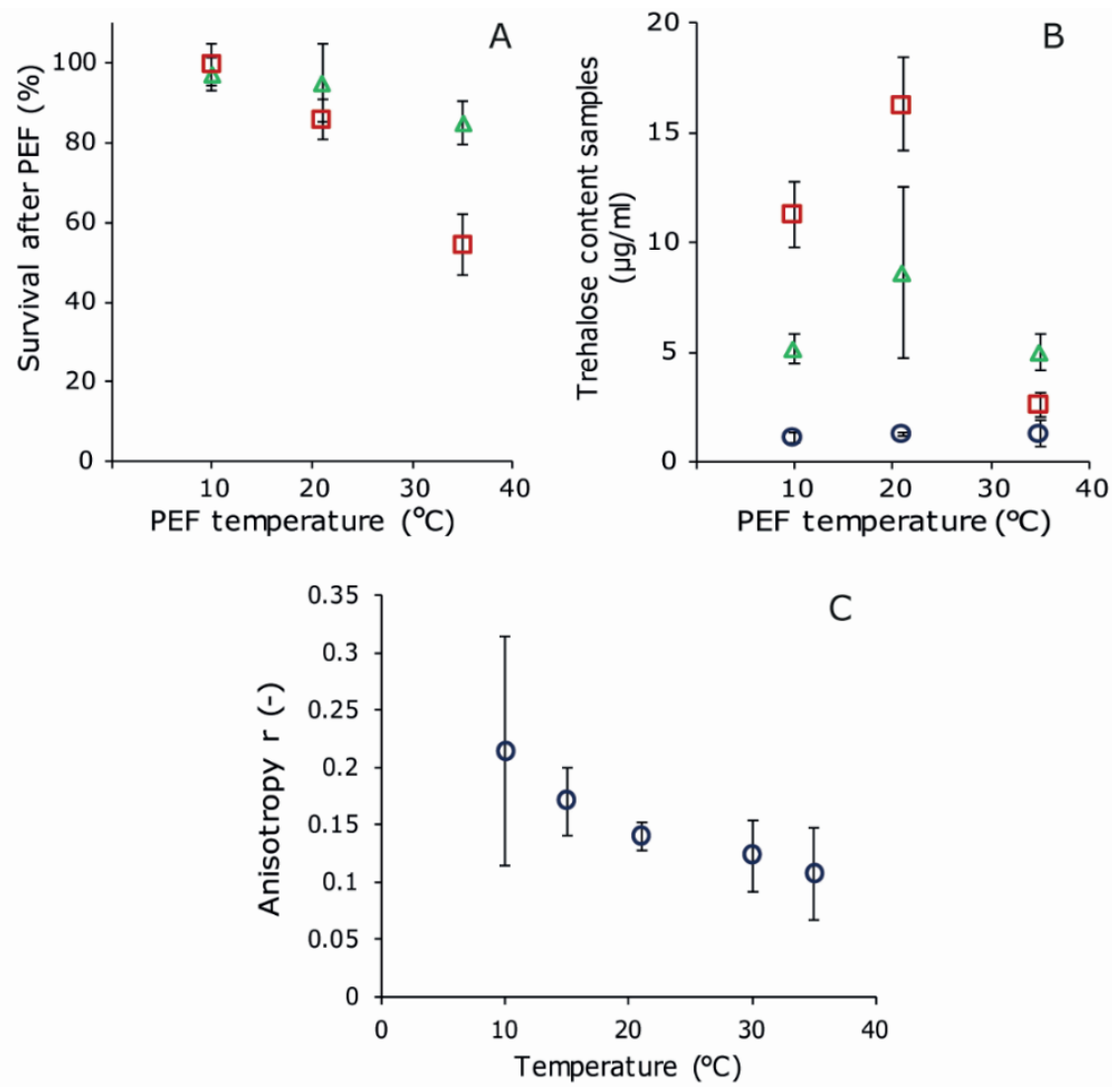

Figure 7.1 Effect of temperature on survival after PEF treatment (A), intracellular trehalose content (B) and membrane fluidity (C) of L. plantarum WCFS1. Different symbols represent control (not PEF treated) (O) and PEF treated cultures with $2(\Delta)$ and $10-12$ pulses $(\square)$ of 100 $\mu$ s at $6.3 \mathrm{kV} / \mathrm{cm}$. Intracellular trehalose contents are presented in $\mu \mathrm{g} / \mathrm{ml}$ sample, with each sample containing $\sim 4 * 10^{9} \mathrm{CFU} / \mathrm{ml}$ before PEF treatment. Panel C shows fluorescence anisotropy values ( $r$ ) determined by DPH analysis. Error bars show standard deviations of biologically independent replicates $(n \geq 3)$.

Not only the temperature can affect the membrane fluidity, also the medium composition can. A component that is widely studied for its effect on membrane fluidity is ethanol (ChuKy et al., 2005; Graca Da Silveira et al., 2003). Model simulations revealed that ethanol can easily pass through the lipid bilayer of cell membrane, leading to a reduced order of the lipid hydrocarbon chains and therefore a higher membrane fluidity (Patra et al., 2006). We studied the effect of the addition of $8 \%(v / v)$ ethanol to the PEF medium to evaluate whether addition of ethanol, which could lead to increased membrane fluidity, has the 
same effect on the outcome of the PEF treatment as performing PEF experiments at a higher temperature. These experiments were carried out at a PEF temperature of $21^{\circ} \mathrm{C}$, similar to all experiments in this thesis except for the experiments with varying temperature that were described before. Unfortunately, we could not measure a difference in fluorescence anisotropy between cultures with and without $8 \%(\mathrm{v} / \mathrm{v})$ ethanol addition, which could be due to the low sensitivity of the DPH method. Remarkably, the addition of $8 \%(\mathrm{v} / \mathrm{v})$ ethanol to the PEF medium resulted in a large increase in intracellular trehalose content after the applied PEF treatments, while survival was similar compared to the cultures without the addition of ethanol (Fig. 7.2). This similar survival after PEF treatment is in agreement with Cebrián et al. (2016), who observed similar survival with and without addition of benzyl alcohol to the PEF medium. If membrane fluidity would be the main factor influencing PEF survival and intracellular trehalose content, we would expect similar results for PEF treatment with ethanol addition as for PEF treatment at higher temperature, since both factors can lead to increased membrane fluidity. However, based on a comparison of these two results, we can conclude that the effect of the temperature and the addition of ethanol on the outcome of the PEF treatment cannot both be explained by their effect on membrane fluidity only.

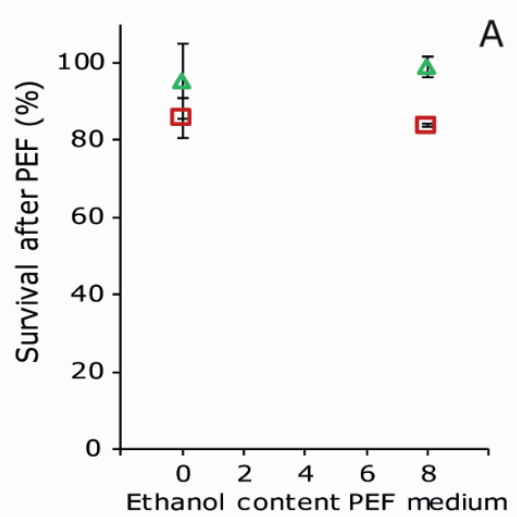

$(\mathrm{v} / \mathrm{v} \%)$

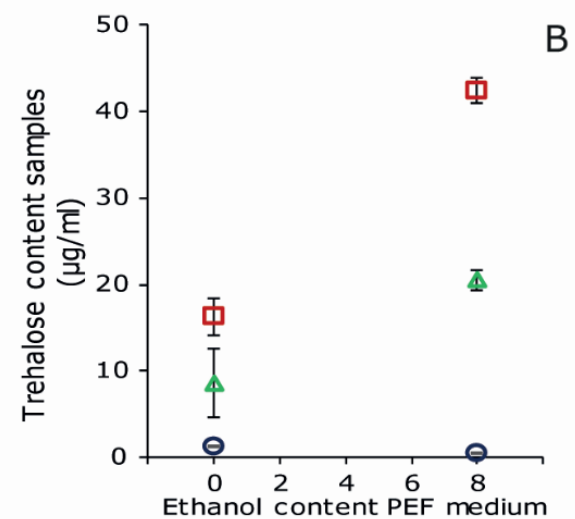

$(\mathrm{v} / \mathrm{v} \%)$

Figure 7.2 Survival (A) and intracellular trehalose content after PEF treatment (B) with and without the addition of $8 \%(\mathrm{~V} / \mathrm{V})$ ethanol to the PEF medium. Control $(\mathbf{O})$ and PEF treated cultures with $2(\Delta)$ and $10-12$ pulses $(\square)$ of $100 \mu \mathrm{s}$ at $6.3 \mathrm{kV} / \mathrm{cm}$. Results at $0 \%(\mathrm{v} / \mathrm{v})$ ethanol are the same results as presented in Fig. 7.1 at $21^{\circ} \mathrm{C}$. Intracellular trehalose contents are presented in $\mu \mathrm{g} / \mathrm{ml}$ sample, with each sample containing $\sim 4 * 10^{9} \mathrm{CFU} / \mathrm{ml}$ before PEF treatment. Error bars show standard deviations of biologically independent replicates $(n=3)$. 


\subsubsection{Strain variability during PEF treatment}

Another factor relevant for evaluation of the use of a PEF pre-treatment in the production process of dried bacteria, is the effect of PEF treatment on different bacterial strains. Survival and intracellular trehalose concentrations after PEF treatment differed between the evaluated bacterial strains (Fig. 7.3). This variability was expected based on literature about the effect of PEF treatments on several bacterial strains (García et al., 2005; Saldaña et al., 2009; Timmermans et al., 2019). When comparing the survival of L. plantarum WCFS1, L. plantarum SF2A35B and L. plantarum LMG23545 we observed that although these strains are from the same species, their survival after a PEF treatment with 2 pulses of $7.5 \mathrm{kV} / \mathrm{cm}$ varied considerably (Fig. 7.3). Actually, these three strains were chosen because of their differences in heat robustness and their observed PEF robustness appeared to be in line with their heat robustness as evaluated by Aryani et al. (2016), with L. plantarum WCFS1 being the most robust strain and $L$. plantarum SF2A35B the least robust. The heat robustness and PEF survival are however not always correlated (Cebrián et al., 2007; Rodríguez-Calleja et al., 2006). Actually, L. lactis FM03 survived very well upon the applied PEF treatment (Fig. 7.3), while this strain was much less resistant to heat compared to $L$. plantarum WCFS1 (Fig. S.5.1).
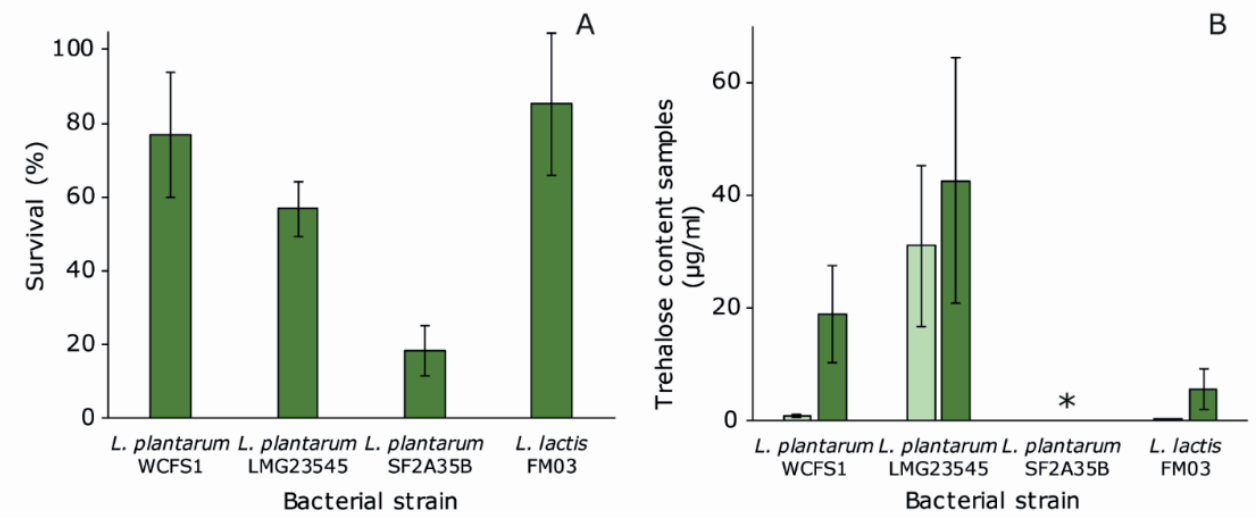

Figure 7.3 Effect of PEF treatment in trehalose electroporation medium with two pulses of $7.5 \mathrm{kV} / \mathrm{cm}$ on survival (A) and intracellular trehalose content (B) of several lactic acid bacteria. Intracellular trehalose contents of control (light green) and PEF treated (green) cultures were corrected based on CFU counts to an initial cell concentration of $4^{*} 10^{9} \mathrm{cfu} / \mathrm{ml}$. Error bars indicate standard deviations of independent replicates $(n \geq 2)$. *Extraction method appeared not to work for L. plantarum SF2A35B because the bead beating procedure to lyse cells was not successful for this strain. 
When comparing intracellular trehalose uptake, it can be observed that intracellular trehalose in L. lactis FM03 increased only slightly using these PEF parameters and for $L$. plantarum LMG23545 already a high initial concentration of intracellular trehalose was found before PEF treatment (Fig. 7.3.B). As shown in chapter 5, higher intracellular trehalose concentrations can be obtained for L. lactis FM03 when applying different PEF conditions. For L. plantarum SF2A35B the trehalose extraction procedure was not working well, probably due to the presence of slimy extracellular polymeric substances, and therefore intracellular components were not extracted after bead beating and no results could be obtained for this strain. Please note that in this strain comparison the differences in cell size and formation of bacterial chains is not taken into account. Larger cell sizes or longer chain lengths per CFU could lead to a higher cell volume per CFU and therefore an improper comparison of concentrations in the cell extracts between different strains in Fig. 7.3B. Our results indicate that PEF treatment can be used to increase intracellular trehalose in different strains, but that for each strain the PEF parameters need to be optimized in order to find the optimal balance between increased intracellular trehalose and PEF survival. Additionally, some of the tested bacteria already contain high intracellular trehalose concentrations after growth, which is further discussed in section 7.4.

\subsection{Intracellular trehalose and other compatible solutes in bacteria}

In addition to evaluation of increased intracellular trehalose upon PEF treatment, we also measured intracellular trehalose in bacteria during pre-culturing. L. plantarum WCFS1 contained high amounts of intracellular trehalose after growth in MRS medium. Indeed, when intracellular trehalose was measured at several time points during growth of $L$. plantarum WCFS1, this revealed that the amount of intracellular trehalose increased greatly during the stationary growth phase (Fig. 7.4). The total intracellular trehalose concentrations after 20 and 24 hours of growth in MRS medium were even much higher than the concentrations obtained after pulsed electric field pre-treatment, i.e. up to 15 $\mu \mathrm{g}^{*} \mathrm{ml}^{-1 *} \mathrm{OD}_{600^{-1}}$, compared to approximately $2 \mu \mathrm{g}^{*} \mathrm{ml}^{-1 *} \mathrm{OD}_{600^{-1}}$. However, in contrast to intracellular trehalose during growth, the increased concentrations of intracellular trehalose due to PEF treatment are probably not present in all individual cells of the population but in a fraction (chapter 2,3 ), so the intracellular concentration in the loaded individual cells might be higher. Interestingly, after suspending the overnight cultures (1618 hours of growth) in trehalose electroporation medium, L. plantarum WCFS1 cultures contained only low concentrations of intracellular trehalose. However, when suspending 
the cultures in lactose electroporation medium, higher intracellular trehalose concentrations were found as described in chapter 4 and 5 . As discussed in these chapters, this could be due to catabolite repression, which is a mechanism involving the repression of the catabolism of one carbohydrate while another preferred carbohydrate is available (Gilbreth et al., 2004; Görke and Stülke, 2008).

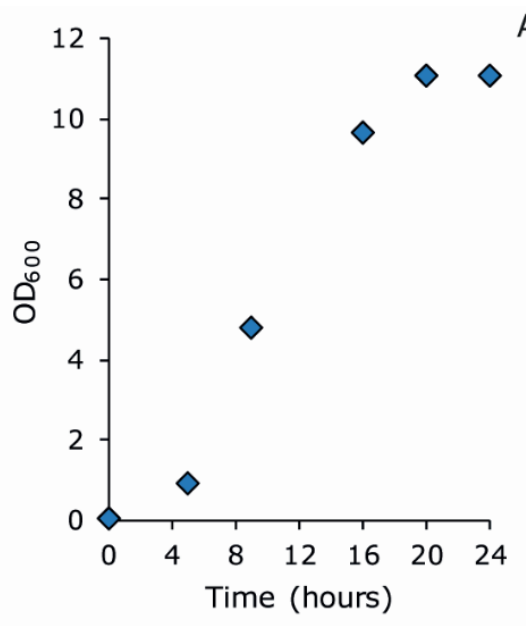

A

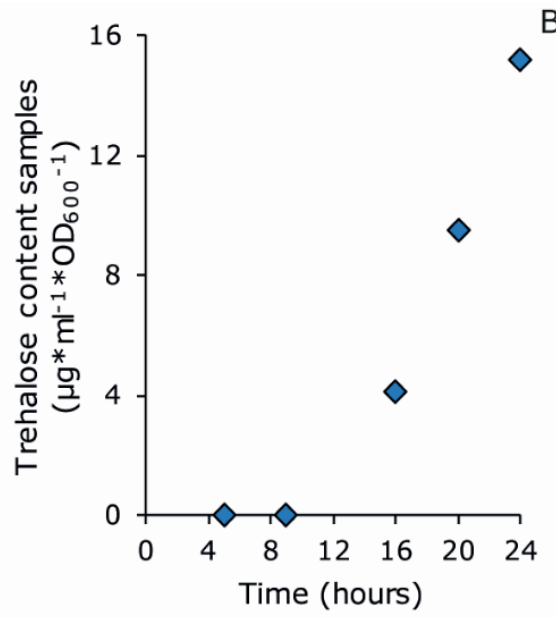

Figure 7.4 $O D_{600}(A)$ and intracellular trehalose content $(B)$ of Lactobacillus plantarum WCFS1 grown in MRS medium. B: Data points at $0 \mathrm{\mu g}^{*} \mathrm{ml}^{-1 *} O D_{600}{ }^{-1}$ were measured, but no trehalose was detected. Data for different time points were obtained from two parallel experiments. Cultures used during all other experiments described in this thesis were grown for 16-18 hours.

These findings triggered the question which biological mechanisms are involved in the intracellular accumulation of trehalose. We used a commercial API 50CH test, which showed that L. plantarum WCFS1 can grow on trehalose as a carbon source (data not shown). Additionally, the growth medium MRS contains yeast extract and therefore probably also trehalose (Plata et al., 2013). This means that L. plantarum WCFS1 can at least take up trehalose from the growth medium and consume it. It could be that this bacterium does not metabolize trehalose due to catabolite repression in the presence of other preferred carbohydrates as explained before. This could explain why trehalose is observed intracellularly after growth of $L$. plantarum WCFS1. Another option would be production of trehalose, which has been described in literature for bacteria such as Propionibacterium freudenreichii, Corynebacterium, Escherichia coli and Mycobactrium (Kaasen et al., 1994; 
Ruhal et al., 2013). The pathways for trehalose production described by Ruhal et al. (2013) are production of trehalose from glucose and glucose-6-P (OtsA and OtsB), production of trehalose from maltodextrin (TreYZ) and the conversion of maltose into trehalose (TreS). Based on a genome blast to find homologues for these genes in L. plantarum WCFS1 it is unlikely that $L$. plantarum WCFS1 contains these pathways for trehalose production. Additionally, upon growing L. plantarum WCFS1 on a chemically defined medium with glucose as a carbon source, and without less defined components that could contain trehalose such as yeast extract, we did not detect any intracellular trehalose after 20 to 24 hours of growth in this medium (data not shown). This chemically defined medium was based on Van Mastrigt et al. (2018), without citrate and with glucose as a carbon source instead of lactose. Detecting intracellular trehalose after growth in a medium that contains yeast extract and not in a medium without yeast extract is in agreement with findings of Lamosa et al. (1998) for a Thermococcus species. This organism is however not closely related to $L$. plantarum as it is classified as an archaea, not as a bacterium.

Intracellular trehalose has also been detected in bacteria that were pre-stressed to osmotic and heat stress before spray drying (Gaucher et al., 2020, 2019). Trehalose was not the only compatible solute that was found intracellularly, also proline, glutamate and glycine betaine. These compatible solutes have also been related before to robustness during drying (Kets et al., 1996) and to osmotic shock responses (Glaasker et al., 1996). Additionally, protective effects of the presence of intracellular calcium in lactic acid bacteria during spray drying have been reported (Wang et al., 2020). In this work we only focused on measurement of intracellular trehalose and in some cases lactose, possible presence of other compatible solutes or stress mechanisms have not been investigated. However, as discussed in chapter 5, the effect of the PEF pre-treatment on survival could not be explained by the amount of intracellular trehalose only. Therefore, insights in what happens with other stress responses and compatible solutes upon the applied PEF treatment might help in further understanding why PEF pre-treatment results in better bacterial survival after spray drying in RSM as a carrier matrix.

\subsection{Implementation of PEF pre-treatment in the production process of dried probiotics and starter cultures}

In chapter five we showed the potential of applying a PEF pre-treatment to increase survival of bacteria during drying and subsequent storage. Implementing this pulsed electric field 
pre-treatment in an industrial scale process requires first of all further understanding of the applicability of this method for different strains, media and pre-culturing methods. Though, upscaling the PEF method is not expected to be a big hurdle in this process, since large scale continuous PEF equipment is already available. Currently, these large scale apparatus for liquid products are mainly used for mild pasteurization purposes in for example fruit juices (Toepfl, 2019). Also, a Swedish start-up company is currently developing PEF processes and equipment leading to increased intracellular protectant concentrations in plant tissues. This PEF pre-treatment is claimed to result in preserved freshness of vegetables, fruit and flower cuttings during shelf-life and can thereby lead to a reduction in waste (OptiFreeze, 2020).

For the implementation of a PEF pre-treatment in the production chain of dried probiotics and starter cultures several options are possible. An interesting option would be performing the PEF pre-treatment in the culture medium with added protectant. However, the high conductivity of culture media such as MRS broth could be a problem. Theoretically, a PEF treatment with 10 pulses of $100 \mu \mathrm{s}$ at $6.3 \mathrm{kV} / \mathrm{cm}$ in growth medium with a conductivity of 1 $\mathrm{S} / \mathrm{m}$ could lead to a maximum temperature increase of $96^{\circ} \mathrm{C}$, based on equation $3.1 \& 3.2$. The specific energy input of the PEF treatment, and thereby the maximum temperature increase, can be decreased by applying less or shorter pulses, lower electric field strengths, or by lowering the medium conductivity. The low conductivity electroporation medium $(0.15 \mathrm{~S} / \mathrm{m})$ used in our study, would result in a maximum temperature increase of $14.5^{\circ} \mathrm{C}$ when applying the same PEF conditions. A large temperature increase is undesired for the purpose of this PEF treatment, therefore it might be more logical to follow a similar approach as described in chapter 5 . Here, the PEF pre-treatment was performed in an electroporation medium and after the PEF pre-treatment, the culture was suspended in a larger volume of carrier matrix (Fig. 7.5, option a). This would require a PEF pre-treatment in a concentrated cell culture. Cell density can affect the efficacy of a PEF treatment (Pucihar et al., 2007). The volume fraction of cells in the electroporation medium during PEF treatment in this thesis was $<0.5 \%$. Pucihar et al. (2007) described a lower cell permeabilization in mammalian cells upon PEF treatment with cell volume fractions of 18 and $36 \%$ compared to $1 \%$. Another option to improve this process would be to re-use the electroporation medium (Fig. 7.5, option b). In this way less electroporation medium is needed, though an extra step (using a.o. centrifugation) is needed to separate the electroporation medium from the cells before suspending the cells in the carrier matrix. 


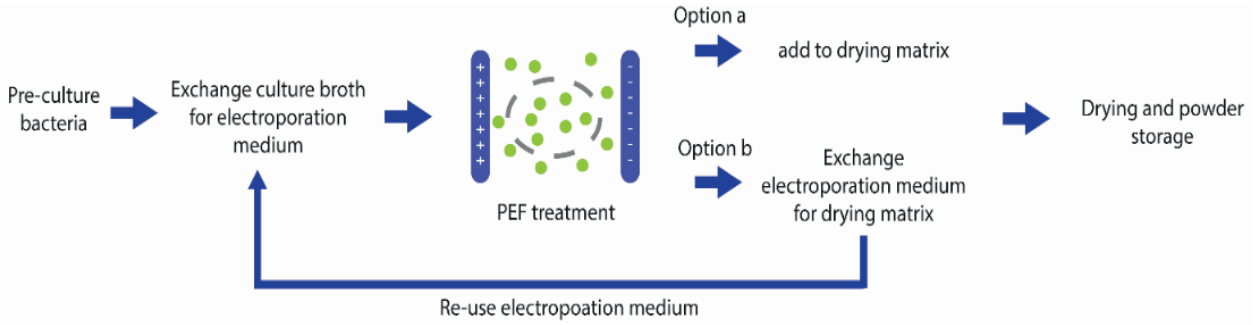

Figure 7.5 Schematic representation of possibilities for implementation of a PEF pretreatment in the production process of dried bacterial ingredients.

Survival after spray drying in RSM was more than doubled when we applied a PEF pretreatment in lactose electroporation medium for L. plantarum WCFS1 (chapter 5). As nowadays many bacterial cultures are freeze dried instead of spray dried because spray drying yields too low survival rates, this increased survival might facilitate a shift from freeze drying to spray drying of these cultures. This shift would lead to a reduction of costs and energy requirement of this production process (Broeckx et al., 2016; Perdana, 2013; Santivarangkna et al., 2007). Perdana (2013) estimated that the energy use for removing 1 $\mathrm{kg}$ water during drying was around $40 \%$ less when shifting from freeze drying to spray drying. Moreover, when we compare the energy required for the additional PEF treatment to the energy required for the subsequent drying, the energy for the PEF treatment is only a minor fraction of the energy needed for the drying process. For example, the specific energy input of the PEF treatment that we applied in chapter 4 and 5 is $57 \mathrm{~kJ} / \mathrm{kg}$ product, while the latent heat of evaporation of water is already $2256 \mathrm{~kJ} / \mathrm{kg}$ water.

\subsection{Conclusions and outlook}

The first part of this thesis focused on the development of a PEF pre-treatment to increase intracellular trehalose concentrations, while maintaining a high culture viability. We have provided the proof of principle that PEF treatment can lead to increased intracellular trehalose concentrations while maintaining a high culture viability in L. plantarum WCFS1. This PEF pre-treatment could be further optimized for future applications in order to achieve higher intracellular trehalose concentrations, for example by adapting the treatment medium. Furthermore, for its application the PEF pre-treatment should be 
further developed for different bacterial strains since the effectivity of a specific PEF treatment can differ significantly between strains.

In the second part of this thesis we focused on the application of this PEF pre-treatment for the production of dried bacterial ingredients. Indeed, we observed that PEF pre-treatment could lead to enhanced survival after spray drying and storage, though this effect was dependent on the carrier matrix. This enhanced survival upon PEF pre-treatment could not be explained by intracellular trehalose and lactose concentrations only. Therefore, further investigation is required to understand which other mechanisms play a role in the protective effect of this PEF pre-treatment and why the protective effect is only found in RSM specifically. This can be done for example by studying the effect of a PEF pre-treatment in trehalose or lactose medium followed by spray drying in carrier matrices based on the composition of RSM, e.g. lactose, minerals and proteins and mixtures of these components. Additional measurements of stress responses such as intracellular accumulation of glycine betaine and glutamate might also help to unravel the underlying mechanism of protection. Furthermore, proteomic and transcriptomic assays can also be useful for this purpose. Overall, this work shows the first steps towards application of a PEF pre-treatment to enhance culture viability after spray drying and subsequent powder storage. 


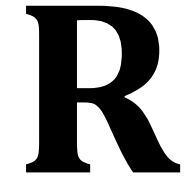

References 
Abe, F., Miyauchi, H., Uchijima, A., Yaeshima, T., Iwatsuki, K., 2009. Effects of storage temperature and water activity on the survival of bifidobacteria in powder form. Int. J. Dairy Technol. 62, 234-239. https://doi.org/10.1111/j.1471-0307.2009.00464.x

Abram, F., Smelt, J.P.P.., Bos, R., Wouters, P.C., 2003. Modelling and optimization of inactivation of Lactobacillus plantarum by pulsed electric field treatment. J. Appl. Microbiol. 94, 571-579. https://doi.org/10.1046/j.1365-2672.2003.01869.x

Agarwal, A., Zudans, I., Weber, E.A., Olofsson, J., Orwar, O., Weber, S.G., 2007. Effect of cell size and shape on single-cell electroporation. Anal. Chem. 79, 3589-3596. https://doi.org/10.1021/ac062049e

Alvarenga, V.O., Brancini, G.T.P., Silva, E.K., da Pia, A.K.R., Campagnollo, F.B., Braga, G.Ú.L., Hubinger, M.D., Sant'Ana, A.S., 2018. Survival variability of 12 strains of Bacillus cereus yielded to spray drying of whole milk. Int. J. Food Microbiol. 286, 80-89. https://doi.org/10.1016/j.ijfoodmicro.2018.07.020

Álvarez, I., Pagán, R., Condón, S., Raso, J., 2003. The influence of process parameters for the inactivation of Listeria monocytogenes by pulsed electric fields. Int. J. Food Microbiol. 87, 87-95. https://doi.org/10.1016/S0168-1605(03)00056-4

Amiali, M., Ngadi, M.O., Smith, J.P., Raghavan, G.S.V., 2007. Synergistic effect of temperature and pulsed electric field on inactivation of Escherichia coli 0157:H7 and Salmonella enteritidis in liquid egg yolk. J. Food Eng. 79, 689-694. https://doi.org/10.1016/j.jfoodeng.2006.02.029

Ananta, E., Volkert, M., Knorr, D., 2005. Cellular injuries and storage stability of spray-dried Lactobacillus rhamnosus GG. Int. Dairy J. 15, 399-409. https://doi.org/10.1016/j.idairyj.2004.08.004

Aronsson, K., Rönner, U., 2001. Influence of $\mathrm{pH}$, water activity and temperature on the inactivation of Escherichia coli and Saccharomyces cerevisiae by pulsed electric fields. Innov. Food Sci. Emerg. Technol. 2, 105-112. https://doi.org/10.1016/S14668564(01)00030-3

Aronsson, K., Rönner, U., Borch, E., 2005. Inactivation of Escherichia coli, Listeria innocua and Saccharomyces cerevisiae in relation to membrane permeabilization and subsequent leakage of intracellular compounds due to pulsed electric field $\begin{array}{lllll}\text { processing. Int. J. Food Microbiol. } & \text { 99, }\end{array}$ https://doi.org/10.1016/j.ijfoodmicro.2004.07.012

Aryani, D.C., den Besten, H.M.W., Zwietering, M.H., 2016. Quantifying variability in growth and thermal inactivation kinetics of Lactobacillus plantarum. Appl. Environ. Microbiol. 82, 4896-4908. https://doi.org/10.1128/AEM.00277-16.Editor 
Asadi, M., 2005. Tables sucrose solutions, in: Beet-Sugar Handbook. John Wiley \& Sons, Inc., pp. 779-801. https://doi.org/10.1002/0471790990

Aschenbrenner, M., Kulozik, U., Foerst, P., 2012. Evaluation of the relevance of the glassy state as stability criterion for freeze-dried bacteria by application of the Arrhenius and WLF model. Cryobiology 65, 308-318. https://doi.org/10.1016/j.cryobiol.2012.08.005

Avaltroni, F., Bouquerand, P.E., Normand, V., 2004. Maltodextrin molecular weight distribution influence on the glass transition temperature and viscosity in aqueous solutions. Carbohydr. Polym. 58, 323-334. https://doi.org/10.1016/j.carbpol.2004.08.001

Barba, F.J., Parniakov, O., Pereira, S.A., Wiktor, A., Grimi, N., Boussetta, N., Saraiva, J.A., Raso, J., Martin-Belloso, O., Witrowa-Rajchert, D., Lebovka, N., Vorobiev, E., 2015. Current applications and new opportunities for the use of pulsed electric fields in food science and industry. Food Res. Int. 77, 773-798. https://doi.org/10.1016/j.foodres.2015.09.015

Bodénès, P., Bensalem, S., Français, O., Pareau, D., Le, B., 2019. Inducing reversible or irreversible pores in Chlamydomonas reinhardtii with electroporation: Impact of treatment parameters. Algal Res. 37, 124-132. https://doi.org/10.1016/j.algal.2018.11.016

Both, E.M., Karlina, A.M., Boom, R.M., Schutyser, M.A.I., 2018. Morphology development during sessile single droplet drying of mixed maltodextrin and whey protein solutions. Food Hydrocoll. 75, 202-210. https://doi.org/10.1016/j.foodhyd.2017.08.022

Both, E.M., Siemons, I., Boom, R.M., Schutyser, M.A.I., 2019a. The role of viscosity in morphology development during single droplet drying. Food Hydrocoll. 94, 510-518. https://doi.org/10.1016/j.foodhyd.2019.03.023

Both, E.M., Tersteeg, S.M.B., Boom, R.M., Schutyser, M.A.I., 2019b. Drying kinetics and viscoelastic properties of concentrated thin films as a model system for spray drying. Colloids Surfaces A Physicochem. Eng. Asp. 585. https://doi.org/10.1016/j.colsurfa.2019.124075

Bouman, J., Venema, P., de Vries, R.J., van der Linden, E., Schutyser, M.A.I., 2016. Hole and vacuole formation during drying of sessile whey protein droplets. Food Res. Int. 84, 128-135. https://doi.org/10.1016/j.foodres.2016.03.027

Bouvier, T., Troussellier, M., Anzil, A., Courties, C., Servais, P., 2001. Using light scatter signal to estimate bacterial biovolume by flow cytometry. Cytometry 44, 188-194. https://doi.org/10.1002/1097-0320(20010701)44:3<188::AID-CYTO1111>3.0.CO;2-C 
Breeuwer, P., Abee, T., 2000. Assessment of viability of microorganisms employing fluorescence techniques. Int. J. Food Microbiol. 55, 193-200. https://doi.org/10.1016/s0168-1605(00)00163-x

Broeckx, G., Kiekens, S., Jokicevic, K., Byl, E., Henkens, T., Vandenheuvel, D., Lebeer, S., Kiekens, F., 2019. Effects of initial cell concentration, growth phase, and process parameters on the viability of Lactobacillus rhamnosus GG after spray drying. Dry. Technol. 0, 1-19. https://doi.org/10.1080/07373937.2019.1648290

Broeckx, G., Vandenheuvel, D., Claes, I.J.J., Lebeer, S., Kiekens, F., 2016. Drying techniques of probiotic bacteria as an important step towards the development of novel $\begin{array}{llll}\text { pharmabiotics. Int. J. } & \text { Pharm. 303-318. }\end{array}$ https://doi.org/10.1016/j.ijpharm.2016.04.002

Broeckx, G., Vandenheuvel, D., Henkens, T., Kiekens, S., van den Broek, M.F.L., Lebeer, S., Kiekens, F., 2017. Enhancing the viability of Lactobacillus rhamnosus GG after spray drying and during storage. Int. J. Pharm. 534, 35-41. https://doi.org/10.1016/j.ijpharm.2017.09.075

Bryant, G., Koster, K.L., Wolfe, J., 2001. Membrane behaviour in seeds and other systems at low water content: The various effects of solutes. Seed Sci. Res. 11, 17-25. https://doi.org/10.1079/SSR200056

Buckow, R., Ng, S., Toepfl, S., 2013. Pulsed electric field processing of orange juice: A review on microbial, enzymatic, nutritional, and sensory quality and stability. Compr. Rev. Food Sci. Food Saf. 12, 455-467. https://doi.org/10.1111/1541-4337.12026

Carvalho, A.L., Cardoso, F.S., Bohn, A., Neves, A.R., Santos, H., 2011. Engineering Trehalose Synthesis in Lactococcus lactis for Improved Stress Tolerance $\square$ †. Appl. Environ. Microbiol. 77, 4189-4199. https://doi.org/10.1128/AEM.02922-10

Castro, N., Durrieu, V., Raynaud, C., Rouilly, A., 2016. Influence of DE-value on the physicochemical properties of maltodextrin for melt extrusion processes. Carbohydr. Polym. 144, 464-473. https://doi.org/10.1016/j.carbpol.2016.03.004

Cebrián, G., Condón, S., Mañas, P., 2016. Influence of growth and treatment temperature on Staphylococcus aureus resistance to pulsed electric fields: Relationship with membrane fluidity. Innov. Food Sci. Emerg. Technol. 37, 161-169. https://doi.org/10.1016/j.ifset.2016.08.011

Cebrián, G., Sagarzazu, N., Pagán, R., Condón, S., Mañas, P., 2007. Heat and pulsed electric field resistance of pigmented and non-pigmented enterotoxigenic strains of Staphylococcus aureus in exponential and stationary phase of growth. Int. J. Food Microbiol. 118, 304-311. https://doi.org/10.1016/j.ijfoodmicro.2007.07.051 
Champagne, C.P., Ross, R.P., Saarela, M., Hansen, K.F., Charalampopoulos, D., 2011. Recommendations for the viability assessment of probiotics as concentrated cultures and in food matrices. Int. J. Food Microbiol. 149, 185-193. https://doi.org/10.1016/j.ijfoodmicro.2011.07.005

Chen, T., Acker, J.P., Eroglu, A., Cheley, S., Bayley, H., Fowler, A., Toner, M., 2001. Beneficial effect of intracellular trehalose on the membrane integrity of dried mammalian cells. Cryobiology 43, 168-181. https://doi.org/10.1006/cryo.2001.2360

Chu-Ky, S., Tourdot-Marechal, R., Marechal, P.A., Guzzo, J., 2005. Combined cold, acid, ethanol shocks in Oenococcus oeni: Effects on membrane fluidity and cell viability. Biochim. Biophys. Acta - Biomembr. 1717, 118-124. https://doi.org/10.1016/j.bbamem.2005.09.015

Corcoran, B.M., Ross, R.P., Fitzgerald, G.F., Stanton, C., 2004. Comparative survival of probiotic lactobacilli spray-dried in the presence of prebiotic substances. J. Appl. Microbiol. 96, 1024-1039. https://doi.org/10.1111/j.1365-2672.2004.02219.x

Corrales, M., Toepfl, S., Butz, P., Knorr, D., Tauscher, B., 2008. Extraction of anthocyanins from grape by-products assisted by ultrasonics, high hydrostatic pressure or pulsed electric fields: A comparison. Innov. Food Sci. Emerg. Technol. 9, 85-91. https://doi.org/10.1016/j.ifset.2007.06.002

Couchman, P.R., Karasz, F.E., 1978. A Classical Thermodynamic Discussion of the Effect of Composition on Glass-Transition Temperatures. Macromolecules 11, 117-119. https://doi.org/10.1021/ma60061a021

Crowe, J.H., Crowe, L.M., Hoekstra, F.A., 1989. Phase Transitions and Permeability Changes in Dry Membranes During Rehydration. J. Bioenerg. Biomembr. 21. https://doi.org/10.1007/BF00762213

Crowe, J.H., Hoekstra, F.A., Crowe, L.M., 1992. Anhydrobiosis. Annu. Rev. Physiol. 54, 579599. https://doi.org/10.1146/annurev.ph.54.030192.003051

Davis, C., 2014. Enumeration of probiotic strains: Review of culture-dependent and alternative techniques to quantify viable bacteria. J. Microbiol. Methods. https://doi.org/10.1016/j.mimet.2014.04.012

Delimaris, J., Tsilimigaki, S., Messini-Nicolaki, N., Ziros, E., Piperakis, S.M., 2006. Effects of pulsed electric fields on DNA of human lymphocytes. Cell Biol. Toxicol. 22, 409-415. https://doi.org/10.1007/s10565-006-0105-1

Demchick, P., Koch, A.L., 1996. The Permeability of the Wall Fabric of Escherichia coli and Bacillus subtilis. J. Bacteriol. 178, 768-773. https://doi.org/10.1128/jb.178.3.768773.1996 
den Besten, H.M.W., Wells-Bennik, M.H.J., Zwietering, M.H., 2018. Natural Diversity in Heat Resistance of Bacteria and Bacterial Spores: Impact on Food Safety and Quality. Annu. Rev. Food Sci. Technol. 9, 383-410. https://doi.org/10.1146/annurev-food-030117012808

Desmond, C., Stanton, C., Fitzgerald, G.F., Collins, K., Paul Ross, R., 2002. Environmental adaptation of probiotic lactobacilli towards improvement of performance during spray drying, in: International Dairy Journal. pp. 183-190. https://doi.org/10.1016/S0958-6946(02)00040-7

Dijkstra, A.R., Setyawati, M.C., Bayjanov, J.R., Alkema, W., Van Hijum, S.A.F.T., Bron, P.A., Hugenholtz, J., 2014. Diversity in robustness of lactococcus lactis strains during heat stress, oxidative stress, and spray drying stress. Appl. Environ. Microbiol. 80, 603-611. https://doi.org/10.1128/AEM.03434-13

Dovgan, B., Barlič, A., Knežević, M., Miklavčič, D., 2017. Cryopreservation of Human Adipose-Derived Stem Cells in Combination with Trehalose and Reversible Electroporation. J. Membr. Biol. 250, 1-9. https://doi.org/10.1007/s00232-016-9916z

Dumont, F., Marechal, P.-A., Gervais, P., 2004. Cell size and water permeability as determining factors for cell viability after freezing at different cooling rates. Appl. Environ. Microbiol. 70, 268-272. https://doi.org/10.1128/AEM.70.1.268

Duong, T., Barrangou, R., Russell, W.M., Klaenhammer, T.R., 2006. Characterization of the tre locus and analysis of trehalose cryoprotection in Lactobacillus acidophilus NCFM. Appl. Environ. Microbiol. 72, 1218-1225. https://doi.org/10.1128/AEM.72.2.12181225.2006

Dymek, K., Dejmek, P., Galindo, F.G., 2014. Influence of Pulsed Electric Field Protocols on the Reversible Permeabilization of Rucola Leaves. Food Bioprocess Technol. 7, 761773. https://doi.org/10.1007/s11947-013-1067-y

Elea, 2020. Elea Technology [WWW Document]. URL https://eleatechnology.de/applications/ (accessed 2.14.20).

Escoffre, J.M., Portet, T., Wasungu, L., Teissié, J., Dean, D., Rols, M.P., 2009. What is (Still not) known of the mechanism by which electroporation mediates gene transfer and expression in cells and tissues. Mol. Biotechnol. 41, 286-295. https://doi.org/10.1007/s12033-008-9121-0

Forssten, S.D., Lahtinen, S.J., Ouwehand, A.C., 2011. The Intestinal Microbiota and Probiotics, in: Malago, J.J., Koninkx, J.F.J.G., Marinsek-Logar, R. (Eds.), Probiotic Bacteria and Enteric Infections: Cytoprotection by Probiotic Bacteria. Springer Netherlands, Dordrecht, pp. 41-63. https://doi.org/10.1007/978-94-007-0386-5_2 
Fox, M.B., Esveld, D.C., Valero, A., Luttge, R., Mastwijk, H.C., Bartels, P. V., Van Den Berg, A., Boom, R.M., 2006. Electroporation of cells in microfluidic devices: A review. Anal. Bioanal. Chem. https://doi.org/10.1007/s00216-006-0327-3

García, D., Gómez, N., Mañas, P., Raso, J., Pagán, R., 2007. Pulsed electric fields cause bacterial envelopes permeabilization depending on the treatment intensity, the treatment medium $\mathrm{pH}$ and the microorganism investigated. Int. J. Food Microbiol. 113, 219-27. https://doi.org/10.1016/j.ijfoodmicro.2006.07.007

García, D., Raso, J., Paga, R., 2005. Bacterial resistance after pulsed electric fields depending on the treatment medium pH. Innov. Food Sci. Emerg. Technol. 6, 388-395. https://doi.org/10.1016/j.ifset.2005.04.003

Garcia, P.A., Ge, Z., Moran, J.L., Buie, C.R., 2016. Microfluidic screening of electric fields for electroporation. Sci. Rep. 6, 21238. https://doi.org/10.1038/srep21238

Gaucher, F., Gagnaire, V., Rabah, H., Maillard, M.B., Bonnassie, S., Pottier, S., Marchand, P., Jan, G., Blanc, P., Jeantet, R., 2019. Taking advantage of bacterial adaptation in order to optimize industrial production of dry propionibacterium freudenreichii. Microorganisms 7. https://doi.org/10.3390/microorganisms7100477

Gaucher, F., Rabah, H., Kponouglo, K., Bonnassie, S., Pottier, S., Dolivet, A., 2020. Intracellular osmoprotectant concentrations determine Propionibacterium freudenreichii survival during drying. Appl. Microbiol. Biotechnol. https://doi.org/10.1007/s00253-020-10425-1

Ghandi, A., Powell, I., Chen, X.D., Adhikari, B., 2012a. Drying kinetics and survival studies of dairy fermentation bacteria in convective air drying environment using single droplet drying. J. Food Eng. 110, 405-417. https://doi.org/10.1016/j.jfoodeng.2011.12.031

Ghandi, A., Powell, I.B., Chen, X.D., Adhikari, B., 2012b. The Effect of Dryer Inlet and Outlet Air Temperatures and Protectant Solids on the Survival of Lactococcus lactis during Spray Drying. Dry. Technol. 30, 1649-1657. https://doi.org/10.1080/07373937.2012.703743

Gilbreth, S.E., Benson, A.K., Hutkins, R.W., 2004. Catabolite repression and virulence gene expression in Listeria monocytogenes. Curr. Microbiol. 49, 95-98. https://doi.org/10.1007/s00284-004-4204-z

Glaasker, E., Konings, W.N., Poolman, B., 1996. Osmotic regulation of intracellular solute pools in Lactobacillus plantarum. J. Bacteriol. 178, 575-582. https://doi.org/10.1128/jb.178.3.575-582.1996

Gong, P., Lin, K., Zhang, J., Han, X., Lyu, L., Yi, H., Sun, J., Zhang, L., 2019. Enhancing spray drying tolerance of Lactobacillus bulgaricus by intracellular trehalose delivery via 
$\begin{array}{llll}\text { electroporation. Food } \quad \text { Res. } & \text { Int. }\end{array}$ https://doi.org/10.1016/j.foodres.2019.108725

Góral, M., Pankiewicz, U., 2017. Effect of Pulsed Electric Fields (PEF) on Accumulation of Magnesium in Lactobacillus rhamnosus B 442 Cells. J. Membr. Biol. 250, 565-572. https://doi.org/10.1007/s00232-017-9986-6

Görke, B., Stülke, J., 2008. Carbon catabolite repression in bacteria: Many ways to make the most out of nutrients. Nat. Rev. Microbiol. 6, 613-624. https://doi.org/10.1038/nrmicro1932

Graca Da Silveira, M., Golovina, E.A., Hoekstra, F.A., Rombouts, F.M., Abee, T., 2003. Membrane Fluidity Adjustments in Ethanol-Stressed Oenococcus oeni Cells. Appl. Environ. Microbiol. 69, 5826-5832. https://doi.org/10.1128/AEM.69.10.5826

Heinz, V., Alvarez, I., Angersbach, A., Knorr, D., 2001. Preservation of liquid foods by high intensity pulsed electric fields-basic concepts for process design. Trends Food Sci. Technol. 12, 103-111. https://doi.org/10.1016/S0924-2244(01)00064-4

Heinz, V., Phillips, S.T., Zenker, M., Knorr, D., 1999. Inactivation of Bacillus subtilis by high intensity pulsed electric fields under close to isothermal conditions. Food Biotechnol. 13, 155-168. https://doi.org/10.1080/08905439909549968

Higl, B., Kurtmann, L., Carlsen, C.U., Ratjen, J., Fo, P., Skibsted, L.H., Kulozik, U., Risbo, J., 2007. Impact of Water Activity, Temperature, and Physical State on the Storage Stability of Lactobacillus paracasei ssp . paracasei Freeze-Dried in a Lactose Matrix. Biotechnol. Prog. 23, 794-800. https://doi.org/10.1021/bp070089d

Hill, C., Guarner, F., Reid, G., Gibson, G.R., Merenstein, D.J., Pot, B., Morelli, L., Canani, R.B., Flint, H.J., Salminen, S., Calder, P.C., Sanders, M.E., 2014. Expert consensus document: The international scientific association for probiotics and prebiotics consensus statement on the scope and appropriate use of the term probiotic. Nat. Rev. Gastroenterol. Hepatol. 11, 506-514. https://doi.org/10.1038/nrgastro.2014.66

Huang, S., Gaucher, F., Cauty, C., Jardin, J., Loir, Y. Le, Jeantet, R., Chen, X.D., Jan, G., 2018. Growth in hyper-concentrated sweet whey triggers multi stress tolerance and spray drying survival in Lactobacillus casei BL23: From the molecular basis to new perspectives for sustainable probiotic production. Front. Microbiol. 9, 1-12. https://doi.org/10.3389/fmicb.2018.02548

Huang, S., Vignolles, M.-L.L., Chen, X.D., Le Loir, Y., Jan, G., Schuck, P., Jeantet, R., Loir, Y. Le, Jan, G., Schuck, P., Jeantet, R., 2017. Spray drying of probiotics and other food-grade bacteria: A review. Trends Food Sci. Technol. 63, 1-17. https://doi.org/10.1016/j.tifs.2017.02.007 
Hülsheger, H., Potel, J., Niemann, E.-G., 1983. Electric field effects on bacteria and yeast cells. Radiat. Environ. Biophys. 22, 149-162. https://doi.org/10.1007/BF01338893

Jaeger, H., Schulz, A., Karapetkov, N., Knorr, D., 2009. Protective effect of milk constituents and sublethal injuries limiting process effectiveness during PEF inactivation of $L b$. rhamnosus. Int. J. Food Microbiol. 134, 154-161. https://doi.org/10.1016/j.ijfoodmicro.2009.06.007

Jahreis, K., Pimentel-Schmitt, E.F., Brückner, R., Titgemeyer, F., 2008. Ins and outs of glucose transport systems in eubacteria. FEMS Microbiol. Rev. 32, 891-907. https://doi.org/10.1111/j.1574-6976.2008.00125.x

Kaasen, I., McDougall, J., Strøm, A.R., 1994. Analysis of the otsBA operon for osmoregulatory trehalose synthesis in Escherichia coli and homology of the OtsA and OtsB proteins to the yeast trehalose-6-phosphate synthase/phosphatase complex. Gene 145, 9-15. https://doi.org/10.1016/0378-1119(94)90316-6

Kalichevsky, M.T., Jaroszkiewicz, E.M., Blanshard, J.M.V., 1993. A study of the glass transition of amylopectin-sugar mixtures. Polymer (Guildf). 34, 346-358. https://doi.org/10.1016/0032-3861(93)90088-R

Kawai, K., Hagiwara, T., Takai, R., Suzuki, T., 2005. Comparative investigation by two analytical approaches of enthalpy relaxation for glassy glucose, sucrose, maltose, and trehalose. Pharm. Res. 22, 490-495. https://doi.org/10.1007/s11095-004-1887-6

Kets, E.P.W., Teunissen, P.J.M., De Bont, J.A.M., 1996. Effect of compatible solutes on survival of lactic acid bacteria subjected to drying. Appl. Environ. Microbiol. 62, 259261.

Khem, S., Small, D.M., May, B.K., 2016. The behaviour of whey protein isolate in protecting Lactobacillus plantarum. Food Chem. 190, 717-723. https://doi.org/10.1016/j.foodchem.2015.06.020

Khem, S., Woo, M.W., Small, D.M., Chen, X.D., May, B.K., 2015. Agent selection and protective effects during single droplet drying of bacteria. Food Chem. 166, 206-214. https://doi.org/10.1016/j.foodchem.2014.06.010

Kleerebezem, M., Binda, S., Bron, P.A., Gross, G., Hill, C., van Hylckama Vlieg, J.E., Lebeer, S., Satokari, R., Ouwehand, A.C., 2019. Understanding mode of action can drive the translational pipeline towards more reliable health benefits for probiotics. Curr. Opin. Biotechnol. 56, 55-60. https://doi.org/10.1016/j.copbio.2018.09.007

Kleerebezem, M., Boekhorst, J., van Kranenburg, R., Molenaar, D., Kuipers, O.P., Leer, R., Tarchini, R., Peters, S.A., Sandbrink, H.M., Fiers, M.W.E.J., Stiekema, W., Lankhorst, R.M.K., Bron, P.A., Hoffer, S.M., Groot, M.N.N., Kerkhoven, R., de Vries, M., Ursing, B., 
de Vos, W.M., Siezen, R.J., 2003. Complete genome sequence of Lactobacillus plantarum WCFS1. PNAS 100, 1990-1995. https://doi.org/10.1073/pnas.0337704100

Koch, A.L., Robertson, B.R., Button, D.K., 1996. Deduction of the cell volume and mass from forward scatter intensity of bacteria analyzed by flow cytometry. J. Microbiol. Methods 27, 49-61. https://doi.org/10.1016/0167-7012(96)00928-1

Kotnik, T., Pucihar, G., Miklavčič, D., 2010. Induced transmembrane voltage and its correlation with electroporation- mediated molecular transport. J. Membr. Biol. 236, 3-13. https://doi.org/10.1007/s00232-010-9279-9

Lamosa, P., Martins, L.O., Da Costa, M.S., Santos, H., 1998. Effects of temperature, salinity, and medium composition on compatible solute accumulation by Thermococcus spp. Appl. Environ. Microbiol. 64, 3591-3598.

Lebaron, P., Catala, P., Parthuisot, N., 1998. Effectiveness of SYTOX green stain for bacterial viability assessment. Appl. Environ. Microbiol. 64, 2697-2700.

Lentz, B.R., 1989. Membrane "fluidity" as detected by diphenylhexatriene probes. Chem. Phys. Lipids 50, 171-190. https://doi.org/10.1016/0009-3084(89)90049-2

Leroy, F., De Vuyst, L., 2004. Lactic acid bacteria as functional starter cultures for the food fermentation industry. Trends Food Sci. Technol. 15, 67-78. https://doi.org/10.1016/j.tifs.2003.09.004

Leslie, S.B., Israeli, E., Lighthart, B., Crowe, J.H., Crowe, L.M., 1995. Trehalose and sucrose protect both membranes and proteins in intact bacteria during drying. Appl. Environ. Microbiol. 61, 3592-3597.

Lian, W.C., Hsiao, H.C., Chou, C.C., 2002. Survival of bifidobacteria after spray-drying. Int. J. Food Microbiol. 74, 79-86. https://doi.org/10.1016/S0168-1605(01)00733-4

Lievense, L.C., Verbeek, M.A.M., Taekema, T., Meerdink, G., Riet, K.V. t., 1992. Modelling the inactivation of Lactobacillus plantarum during a drying process. Chem. Eng. Sci. 47, 87-97. https://doi.org/10.1016/0009-2509(92)80203-0

Liu, B., Fu, N., Woo, M.W., Chen, X.D., 2018. Heat stability of Lactobacillus rhamnosus GG and its cellular membrane during droplet drying and heat treatment. Food Res. Int. 112, 56-65. https://doi.org/10.1016/j.foodres.2018.06.006

Loghavi, L., Sastry, S.K., Yousef, A.E., 2008. Effect of moderate electric field frequency on growth kinetics and metabolic activity of Lactobacillus acidophilus. Biotechnol. Prog. 24, 148-153. https://doi.org/10.1021/bp070268v

Lopez, N., Puertolas, E., Condon, S., Raso, J., Alvarez, I., 2009. Enhancement of the extraction of betanine from red beetroot by pulsed electric fields. J. Food Eng. 90, 60-66. 
Luby-Phelps, K., 1999. Cytoarchitecture and physical properties of cytoplasm: volume, viscosity, diffusion, intracellular surface area. Int. Rev. Cytol. 192, 189-221. https://doi.org/10.1016/S0074-7696(08)60527-6

Meneghel, J., Passot, S., Cenard, S., Réfrégiers, M., Jamme, F., Fonseca, F., 2017. Subcellular membrane fluidity of Lactobacillus delbrueckii subsp. bulgaricus under cold and osmotic stress. Appl. Microbiol. Biotechnol. 101, 6907-6917. https://doi.org/10.1007/s00253-017-8444-9

Meng, X.C., Stanton, C., Fitzgerald, G.F., Daly, C., Ross, R.P., 2008. Anhydrobiotics: The challenges of drying probiotic cultures. Food Chem. 106, 1406-1416. https://doi.org/10.1016/j.foodchem.2007.04.076

Mensink, M.A., Frijlink, H.W., Voort, K. Van Der, Hinrichs, W.L.J., 2017. How sugars protect proteins in the solid state and during drying ( review ): Mechanisms of stabilization in relation to stress conditions. Eur. J. Pharm. Biopharm. 114, 288-295. https://doi.org/10.1016/j.ejpb.2017.01.024

Miklavčič, D., Serša, G., Brecelj, E., Gehl, J., Soden, D., Bianchi, G., Ruggieri, P., Rossi, C.R., Campana, L.G., Jarm, T., 2012. Electrochemotherapy: technological advancements for efficient electroporation-based treatment of internal tumors. Med. Biol. Eng. Comput. 50, 1213-1225. https://doi.org/10.1007/s11517-012-0991-8

Mille, Y., Obert, J.P., Beney, L., Gervais, P., 2004. New drying process for lactic bacteria based on their dehydration behavior in liquid medium. Biotechnol. Bioeng. 88, 71-76. https://doi.org/10.1002/bit.20211

Müller, S., Nebe-Von-Caron, G., 2010. Functional single-cell analyses: Flow cytometry and cell sorting of microbial populations and communities. FEMS Microbiol. Rev. 34, 554587. https://doi.org/10.1111/j.1574-6976.2010.00214.x

Mykytczuk, N.C.S., Trevors, J.T., Leduc, L.G., Ferroni, G.D., 2007. Fluorescence polarization in studies of bacterial cytoplasmic membrane fluidity under environmental stress. $\begin{array}{llll}\text { Prog. Biophys. Mol. } & \text { Biol. }\end{array}$ https://doi.org/10.1016/j.pbiomolbio.2007.05.001

Nagpal, R., Kumar, A., Kumar, M., Behare, P. V., Jain, S., Yadav, H., 2012. Probiotics, their health benefits and applications for developing healthier foods: A review. FEMS Microbiol. Lett. 334, 1-15. https://doi.org/10.1111/j.1574-6968.2012.02593.x

Najim, N., Aryana, K.J., 2013. A mild pulsed electric field condition that improves acid tolerance, growth, and protease activity of Lactobacillus acidophilus LA-K and Lactobacillus delbrueckii subspecies bulgaricus LB-12. J. Dairy Sci. 96, 3424-3434. 
https://doi.org/10.3168/jds.2012-5842

Nicolai, T., Britten, M., Schmitt, C., 2011. $\beta$-Lactoglobulin and WPI aggregates: Formation, structure and applications. Food Hydrocoll. 25, 1945-1962. https://doi.org/10.1016/j.foodhyd.2011.02.006

OptiFreeze, 2020. Optifreeze technology [WWW Document]. URL https://optifreeze.se/technology/ (accessed 1.29.20).

Pankiewicz, U., Góral, M., Kozłowicz, K., Góral, D., 2020. Application of pulsed electric field in production of ice cream enriched with probiotic bacteria (L. rhamnosus B 442) containing intracellular calcium ions. J. Food Eng. https://doi.org/10.1016/j.jfoodeng.2019.109876

Paramita, V., lida, K., Yoshii, H., Furuta, T., 2010. Effect of additives on the morphology of spray-dried powder. Dry. Technol. 28, 323-329. https://doi.org/10.1080/07373931003627098

Pataro, G., Senatore, B., Donsì, G., Ferrari, G., 2011. Effect of electric and flow parameters on PEF treatment efficiency. J. Food Eng. 105, 79-88. https://doi.org/10.1016/j.jfoodeng.2011.02.007

Patra, M., Salonen, E., Terama, E., Vattulainen, I., Faller, R., Lee, B.W., Holopainen, J., Karttunen, M., 2006. Under the influence of alcohol: The effect of ethanol and methanol on lipid bilayers. Biophys. J. 90, 1121-1135. https://doi.org/10.1529/biophysj.105.062364

Peighambardoust, S.H., Golshan Tafti, A., Hesari, J., 2011. Application of spray drying for preservation of lactic acid starter cultures: A review. Trends Food Sci. Technol. https://doi.org/10.1016/j.tifs.2011.01.009

Pénicaud, C., Monclus, V., Perret, B., Passot, S., Fonseca, F., 2018. Life cycle assessment of the production of stabilized lactic acid bacteria for the environmentally-friendly preservation of living cells. J. Clean. Prod. 184, 847-858. https://doi.org/10.1016/j.jclepro.2018.02.191

Perdana, J., 2013. Single droplet analysis for spray drying of foods. PhD thesis, Wageningen University.

Perdana, J., Bereschenko, L., Fox, M.B., Kuperus, J.H., Kleerebezem, M., Boom, R.M., Schutyser, M.A.I., 2013. Dehydration and thermal inactivation of Lactobacillus plantarum WCFS1: Comparing single droplet drying to spray and freeze drying. Food Res. Int. 54, 1351-1359. https://doi.org/10.1016/j.foodres.2013.09.043

Perdana, J., Bereschenko, L., Roghair, M., Fox, M.B., Boom, R.M., Kleerebezem, M., Schutyser, M.A.I., 2012. Novel method for enumeration of viable Lactobacillus 
plantarum WCFS1 cells after single-droplet drying. Appl. Environ. Microbiol. 78, 80828088. https://doi.org/10.1128/AEM.02063-12

Perdana, J., den Besten, H.M.W., Aryani, D.C., Kutahya, O., Fox, M.B., Kleerebezem, M., Boom, R.M., Schutyser, M.A.I., 2014a. Inactivation of Lactobacillus plantarum WCFS1 during spray drying and storage assessed with complementary viability determination methods. Food Res. Int. 64, 212-217. https://doi.org/10.1016/j.foodres.2014.06.029

Perdana, J., Fox, M.B., Siwei, C., Boom, R.M., Schutyser, M.A.I., 2014b. Interactions between formulation and spray drying conditions related to survival of Lactobacillus plantarum WCFS1. Food Res. Int. 56, 9-17. https://doi.org/10.1016/j.foodres.2013.12.007

Phoon, P.Y., Go, F., Vicente, A., Dejmek, P., 2008. Pulsed electric field in combination with vacuum impregnation with trehalose improves the freezing tolerance of spinach leaves. J. Food Eng. 88, 144-148. https://doi.org/10.1016/j.jfoodeng.2007.12.016

Pillet, F., Formosa-dague, C., Baaziz, H., Dague, E., 2016. Cell wall as a target for bacteria inactivation by pulsed electric fields. Sci. Rep. 6. https://doi.org/10.1038/srep19778

Plata, M.R., Koch, C., Wechselberger, P., Herwig, C., Lendl, B., 2013. Determination of carbohydrates present in Saccharomyces cerevisiae using mid-infrared spectroscopy and partial least squares regression. Anal. Bioanal. Chem. 405, 8241-8250. https://doi.org/10.1007/s00216-013-7239-9

Pucihar, G., Kotnik, T., Teissié, J., Miklavčič, D., 2007. Electropermeabilization of dense cell suspensions. Eur. Biophys. J. 36, 173-185. https://doi.org/10.1007/s00249-006-01151

Pulsemaster, 2020. Pulsemaster [WWW Document]. URL https://www.pulsemaster.us/pefproducts (accessed 2.14.20).

Raso, J., Frey, W., Ferrari, G., Pataro, G., Knorr, D., Teissie, J., Miklavčič, D., 2016. Recommendations guidelines on the key information to be reported in studies of application of PEF technology in food and biotechnological processes. Innov. Food Sci. Emerg. Technol. 37, 312-321. https://doi.org/10.1016/j.ifset.2016.08.003

Ribeiro, C., Zimeri, J.E., Yildiz, E., Kokini, J.L., 2002. Estimation of effective diffusivities and glass transition temperature of polydextrose as a function of moisture content. Carbohydr. Polym. 51, 273-280. https://doi.org/10.1016/S0144-8617(02)00182-0

Rodríguez-Calleja, J.M., Cebrián, G., Condón, S., Mañas, P., 2006. Variation in resistance of natural isolates of Staphylococcus aureus to heat, pulsed electric field and ultrasound under pressure. J. Appl. Microbiol. 100, 1054-1062. https://doi.org/10.1111/j.13652672.2006.02868.x

Rols, M.P., Teissié, J., 1990. Electropermeabilization of mammalian cells. Quantitative 
analysis of the phenomenon. Biophys. J. 58, 1089-1098. https://doi.org/10.1016/S0006-3495(90)82451-6

Ruhal, R., Kataria, R., Choudhury, B., 2013. Trends in bacterial trehalose metabolism and significant nodes of metabolic pathway in the direction of trehalose accumulation. Microb. Biotechnol. 6, 493-502. https://doi.org/10.1111/1751-7915.12029

Sadek, C., Li, H., Schuck, P., Fallourd, Y., Pradeau, N., Le Floch-Fouéré, C., Jeantet, R., 2014. To what extent do whey and casein micelle proteins influence the morphology and properties of the resulting powder? Dry. Technol. 32, 1540-1551. https://doi.org/10.1080/07373937.2014.915554

Sadek, C., Tabuteau, H., Schuck, P., Fallourd, Y., Pradeau, N., Le Floch-Fouéré, C., Jeantet, R., 2013. Shape, shell, and vacuole formation during the drying of a single concentrated whey protein droplet. Langmuir 29, 15606-15613. https://doi.org/10.1021/la404108v

Saldaña, G., Puértolas, E., López, N., García, D., Álvarez, I., Raso, J., 2009. Comparing the PEF resistance and occurrence of sublethal injury on different strains of Escherichia coli, Salmonella Typhimurium, Listeria monocytogenes and Staphylococcus aureus in media of $\mathrm{pH} 4$ and 7. Innov. Food Sci. Emerg. Technol. 10, 160-165. https://doi.org/https://doi.org/10.1016/j.ifset.2008.11.003

Sanders, M.E., Benson, A., Lebeer, S., Merenstein, D.J., Klaenhammer, T.R., 2018. Shared mechanisms among probiotic taxa: implications for general probiotic claims. Curr. Opin. Biotechnol. 49, 207-216. https://doi.org/10.1016/j.copbio.2017.09.007

Santivarangkna, C., Aschenbrenner, M., Kulozik, U., Foerst, P., 2011. Role of Glassy State on Stabilities of Freeze-Dried Probiotics. J. Food Sci. 76. https://doi.org/10.1111/j.17503841.2011.02347.x

Santivarangkna, C., Higl, B., Foerst, P., 2008. Protection mechanisms of sugars during different stages of preparation process of dried lactic acid starter cultures. Food Microbiol. 25, 429-441. https://doi.org/10.1016/j.fm.2007.12.004

Santivarangkna, C., Kulozik, U., Foerst, P., 2007. Alternative drying processes for the industrial preservation of lactic acid starter cultures. Biotechnol. Prog. 23, 302-315. https://doi.org/10.1021/bp060268f

Saulis, G., 2010. Electroporation of cell membranes: the fundamental effects of pulsed electric fields in food processing. Food Eng. Rev. 2, 52-73. https://doi.org/10.1007/s12393-010-9023-3

Schill, R.O., Mali, B., Dandekar, T., Schnölzer, M., Reuter, D., Frohme, M., 2009. Molecular mechanisms of tolerance in tardigrades: New perspectives for preservation and 
stabilization of biological material. Biotechnol. Adv. 27, 348-352. https://doi.org/10.1016/j.biotechadv.2009.01.011

Schutyser, M.A.I., Both, E.M., Siemons, I., Vaessen, E.M.J., Zhang, L., 2019. Gaining insight on spray drying behavior of foods via single droplet drying analyses. Dry. Technol. 37, 525-534. https://doi.org/10.1080/07373937.2018.1482908

Schutyser, M.A.I., Perdana, J., Boom, R.M., 2012. Single droplet drying for optimal spray drying of enzymes and probiotics. Trends Food Sci. Technol. https://doi.org/10.1016/j.tifs.2012.05.006

Sender, R., Fuchs, S., Milo, R., 2016. Are we really vastly outnumbered? Revisiting the ratio of bacterial to host cells in humans. Cell 164, 337-340. https://doi.org/10.1016/j.cell.2016.01.013

Shi, J., Ma, Y., Zhu, J., Chen, Y., Sun, Y., Yao, Y., 2018. A review on electroporation-based intracellular delivery. Molecules 23. https://doi.org/10.3390/molecules23113044

Shirakashi, R., Köstner, C.M., Müller, K.J., Kürschner, M., Zimmermann, U., Sukhorukov, V.L., 2002. Intracellular delivery of trehalose into mammalian cells by electropermeabilization. J. Membr. Biol. 189, 45-54. https://doi.org/10.1007/s00232-002-1003-y

Shirakashi, R., Sukhorukov, V.L., Tanasawa, I., Zimmermann, U., 2004. Measurement of the permeability and resealing time constant of the electroporated mammalian cell membranes. Int. J. Heat Mass Transf. 47, 4517-4524. https://doi.org/10.1016/j.ijheatmasstransfer.2004.04.007

Siemons, I., Politiek, R.G.A., Boom, R.M., Sman, R.G.M. Van Der, Schutyser, M.A.I., 2020a. Dextrose equivalence of maltodextrins determines particle morphology development during single sessile droplet drying. Food Res. Int. 131, 108988. https://doi.org/10.1016/j.foodres.2020.108988

Siemons, I., Vaessen, E.M.J., Oosterbaan van Peski, S.E., Boom, R.M., Schutyser, M.A.I., 2021. Protective effect of carrier matrices on survival of Lactobacillus plantarum WCFS1 during single droplet drying explained by particle morphology development. J. Food Eng., 292, 110263.

Silve, A., Leray, I., Poignard, C., Mir, L.M., 2016. Impact of external medium conductivity on cell membrane electropermeabilization by microsecond and nanosecond electric pulses. Sci. Rep. 6, 19957. https://doi.org/10.1038/srep19957

Siragusa, S., De Angelis, M., Calasso, M., Campanella, D., Minervini, F., Di Cagno, R., Gobbetti, M., 2014. Fermentation and proteome profiles of Lactobacillus plantarum strains during growth under food-like conditions. J. Proteomics 96, 366-380. 
https://doi.org/10.1016/j.jprot.2013.11.003

Soukoulis, C., Behboudi-Jobbehdar, S., Yonekura, L., Parmenter, C., Fisk, I., 2014. Impact of Milk Protein Type on the Viability and Storage Stability of Microencapsulated Lactobacillus acidophilus NCIMB 701748 Using Spray Drying. Food Bioprocess Technol. 7, 1255-1268. https://doi.org/10.1007/s11947-013-1120-x

Stewart, S., He, X., 2019. Intracellular Delivery of Trehalose for Cell Banking. Langmuir 35, 7414-7422. https://doi.org/10.1021/acs.langmuir.8b02015

Stocks, S.M., 2004. Mechanism and use of the commercially available viability stain, BacLight. Cytom. Part A 61, 189-195. https://doi.org/10.1002/cyto.a.20069

Sträuber, H., Müller, S., 2010. Viability states of bacteria-Specific mechanisms of selected probes. Cytom. Part A 77, 623-634. https://doi.org/10.1002/cyto.a.20920

Tapia, H., Young, L., Fox, D., Bertozzi, C.R., Koshland, D., 2015. Increasing intracellular trehalose is sufficient to confer desiccation tolerance to Saccharomyces cerevisiae. PNAS 112, 6122-6127. https://doi.org/10.1073/pnas.1506415112

Teissie, J., Golzio, M., Rols, M.P., 2005. Mechanisms of cell membrane electropermeabilization: A minireview of our present (lack of ?) knowledge. Biochim. Biophys. Acta - Gen. Subj. https://doi.org/10.1016/j.bbagen.2005.05.006

Teissié, J., Rols, M.P., 1993. An experimental evaluation of the critical potential difference inducing cell membrane electropermeabilization. Biophys. J. 65, 409-413. https://doi.org/10.1016/S0006-3495(93)81052-X

Termont, S., Vandenbroucke, K., Iserentant, D., Neirynck, S., Steidler, L., Remaut, E., Rottiers, P., 2006. Intracellular accumulation of trehalose protects Lactococcus lactis from freeze-drying damage and bile toxicity and increases gastric acid resistance. Appl. Environ. Microbiol. 72, 7694-7700. https://doi.org/10.1128/AEM.01388-06

Timmermans, R.A.H., 2018. Moderate and high intensity pulsed electric fields : effect on microbial inactivation, shelf life and quality of fruit juices. PhD thesis, Wageningen University. https://doi.org/10.8174/426533

Timmermans, R.A.H., Mastwijk, H.C., Berendsen, L.B.J.M., Nederhoff, A.L., Matser, A.M., Van Boekel, M.A.J.S., Nierop Groot, M.N., 2019. Moderate intensity Pulsed Electric Fields (PEF) as alternative mild preservation technology for fruit juice. Int. J. Food Microbiol. 298, 63-73. https://doi.org/10.1016/j.ijfoodmicro.2019.02.015

Timmermans, R.A.H., Nierop Groot, M.N., Nederhoff, A.L., van Boekel, M.A.J.S., Matser, A.M., Mastwijk, H.C., 2014. Pulsed electric field processing of different fruit juices: Impact of $\mathrm{pH}$ and temperature on inactivation of spoilage and pathogenic microorganisms. Int. J. Food Microbiol. 173, 105-111. 
https://doi.org/10.1016/j.ijfoodmicro.2013.12.022

Toepfl, S., 2019. Use of Pulsed Electric Fields (PEF) in the food industry [WWW Document]. foodhubmagazine.com. URL https://www.foodhubmagazine.com/2019/08/31/useof-pulsed-electric-fields-pef-in-the-food-industry/ (accessed 10.22.19).

Toepfl, S., Heinz, V., Knorr, D., 2007. High intensity pulsed electric fields applied for food preservation. Chem. Eng. Process. Process Intensif. 46, 537-546. https://doi.org/10.1016/j.cep.2006.07.011

Toepfl, S., Heinz, V., Knorr, D., 2005. Overview of pulsed electric field processing of foods, in: Emerging Technologies for Food Processing. Academic Press: London, UK, pp. 6997.

Tryfona, T., Bustard, M.T., 2008. Impact of pulsed electric fields on Corynebacterium glutamicum cell membrane permeabilization. J. Biosci. Bioeng. 105, 375-382. https://doi.org/10.1263/jbb.105.375

Tsong, T.Y., 1991. Electroporation of cell membranes. Biophys. J. 60, 297-306. https://doi.org/10.1016/S0006-3495(91)82054-9

Ulmer, H.M., Heinz, V., Gänzle, M.G., Knorr, D., Vogel, R.F., 2002. Effects of pulsed electric fields on inactivation and metabolic activity of Lactobacillus plantarum in model beer. J. Appl. Microbiol. 93, 326-335. https://doi.org/10.1046/j.1365-2672.2002.01699.x

Unal, R., Yousef, A.E., Dunne, C.P., 2002. Spectrofluorimetric assessment of bacterial cell membrane damage by pulsed electric field. Innov. Food Sci. Emerg. Technol. 3, 247254. https://doi.org/10.1016/S1466-8564(02)00033-4

Vaessen, E.M.J., den Besten, H.M.W., Esveld, E.D.C., Schutyser, M.A.I., 2019. Accumulation of intracellular trehalose and lactose in Lactobacillus plantarum WCFS1 during pulsed electric field treatment and subsequent freeze and spray drying. LWT 115, 108478. https://doi.org/10.1016/j.Iwt.2019.108478

Vaessen, E.M.J., den Besten, H.M.W., Patra, T., van Mossevelde, N.T.M., Boom, R.M., Schutyser, M.A.I., 2018. Pulsed electric field for increasing intracellular trehalose content in Lactobacillus plantarum WCFS1. Innov. Food Sci. Emerg. Technol. 47, 256261. https://doi.org/10.1016/j.ifset.2018.03.007

Vaessen, E.M.J., Leito, K.M.N., den Besten, H.M.W., Schutyser, M.A.I., 2020. Pulsed electric field pre-treatment for enhanced bacterial robustness during drying: Effect of carrier matrix and strain variability. Manuscr. Prep.

van den Nieuwboer, M., van Hemert, S., Claassen, E., de Vos, W.M., 2016. Lactobacillus plantarum WCFS1 and its host interaction: a dozen years after the genome. Microb. Biotechnol. 9, 452-465. https://doi.org/10.1111/1751-7915.12368 
van der Sman, R.G.M., Meinders, M.B.J., 2013. Moisture diffusivity in food materials. Food Chem. 138, 1265-1274. https://doi.org/10.1016/j.foodchem.2012.10.062

van der Sman, R.G.M., Meinders, M.B.J., 2011. Prediction of the state diagram of starch water mixtures using the Flory-Huggins free volume theory. Soft Matter 7, 429-442. https://doi.org/10.1039/c0sm00280a

van Mastrigt, O., Abee, T., Smid, E.J., 2017. Complete genome sequences of Lactococcus lactis subsp. lactis bv. diacetylactis FM03 and Leuconostoc mesenteroides FM06 isolated from cheese. Genome Announc. 5:e00633-1. https://doi.org/10.1128/genomeA.00633-17

van Mastrigt, O., Mager, E.E., Jamin, C., Abee, T., Smid, E.J., 2018. Citrate, low pH and amino acid limitation induce citrate utilization in Lactococcus lactis biovar diacetylactis. Microb. Biotechnol. 11, 369-380. https://doi.org/10.1111/1751-7915.13031

Vehring, R., 2008. Pharmaceutical particle engineering via spray drying. Pharm. Res. 25, 999-1022. https://doi.org/10.1007/s11095-007-9475-1

Vehring, R., Foss, W.R., Lechuga-Ballesteros, D., 2007. Particle formation in spray drying. J. Aerosol Sci. 38, 728-746. https://doi.org/10.1016/j.jaerosci.2007.04.005

Wang, J., Huang, S., Fu, N., Jeantet, R., Chen, X.D., 2016. Thermal aggregation of calciumfortified skim milk enhances probiotic protection during convective droplet drying. J. Agric. Food Chem. 64, 6003-6010. https://doi.org/10.1021/acs.jafc.6b02205

Wang, Q., Li, Y., Sun, D., Zhu, Z., 2018. Enhancing food processing by pulsed and high voltage electric fields : principles and applications. Crit. Rev. Food Sci. Nutr. 58, 2285-2298. https://doi.org/10.1080/10408398.2018.1434609

Wang, Y., Hao, F., Lu, W., Suo, X., Bellenger, E., Fu, N., Jeantet, R., Chen, X.D., 2020. Enhanced thermal stability of lactic acid bacteria during spray drying by intracellular accumulation of calcium. J. Food Eng. 109975. https://doi.org/10.1016/J.JFOODENG.2020.109975

Wouters, P., Alvarez, I., Raso, J., 2001a. Critical factors determining inactivation kinetics by pulsed electric field food processing. Trends Food Sci. Technol. 12, 112-121. https://doi.org/10.1016/S0924-2244(01)00067-X

Wouters, P., Bos, A.P., Ueckert, J., 2001b. Membrane permeabilization in relation to inactivation kinetics of Lactobacillus species due to pulsed electric fields. Appl. Environ. Microbiol. 67, 3092-3101. https://doi.org/10.1128/AEM.67.7.3092

Würth, R., Foerst, P., Kulozik, U., 2018. Effects of skim milk concentrate dry matter and spray drying air temperature on formation of capsules with varying particle size and the survival microbial cultures in a microcapsule matrix. Dry. Technol. 36, 93-99. 
https://doi.org/10.1080/07373937.2017.1301952

Yang, Z., Peng, H., Wang, W., Liu, T., 2010. Crystallization behavior of poly( $\varepsilon$ caprolactone)/layered double hydroxide nanocomposites. J. Appl. Polym. Sci. 116, 2658-2667. https://doi.org/10.1002/app

Yaqub, S., Anderson, J.G., Macgregor, S.J., Rowan, N.J., 2004. Use of a fluorescent viability stain to assess lethal and sublethal injury in food-borne bacteria exposed to highintensity pulsed electric fields. Lett. Appl. Microbiol. 246-251. https://doi.org/10.1111/j.1472-765X.2004.01571.x

Zhang, Q., Yan, T., 2012. Correlation of intracellular trehalose concentration with desiccation resistance of soil Escherichia coli populations. Appl. Environ. Microbiol. 78, 7407-7413. https://doi.org/10.1128/AEM.01904-12

Zheng, X., Fu, N., Duan, M., Woo, M.W., Selomulya, C., Chen, X.D., 2015. The mechanisms of the protective effects of reconstituted skim milk during convective droplet drying of lactic acid bacteria. Food Res. Int. 76, 478-488. https://doi.org/https://doi.org/10.1016/j.foodres.2015.07.045

Zheng, X., Fu, N., Huang, S., Jeantet, R., Chen, X.D., 2016. Exploring the protective effects of calcium-containing carrier against drying-induced cellular injuries of probiotics using single droplet drying technique. Food Res. Int. 90, 226-234. https://doi.org/10.1016/j.foodres.2016.10.034

Zimmermann, U., Pilwat, G., Riemann, F., 1974. Dielectric Breakdown of Cell Membranes. Biophys. J. 14, 881-899. https://doi.org/10.1016/S0006-3495(74)85956-4

Zobrist, B., Soonsin, V., Luo, B.P., Krieger, U.K., Marcolli, C., Peter, T., Koop, T., 2011. Ultraslow water diffusion in aqueous sucrose glasses. Phys. Chem. Chem. Phys. 13, 35143526. https://doi.org/10.1039/c0cp01273d 



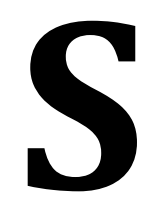

Summary

Samenvatting 


\section{Summary}

Bacteria are commonly used in food industry as starter cultures or probiotic supplements. Production of these bacterial food ingredients requires growth of the bacteria followed by storage until they are used as fermentation starter or ingested as probiotic. To prolong the shelf life of these bacteria, drying processes such as freeze drying and spray drying are used to dry these ingredients into a powder. A drawback of drying is that the viability of the dried bacterial cultures decrease along the process chain. Therefore, many studies focus on improving the survival of these cultures during drying and subsequent storage. Often the bacteria are dried in a carrier matrix consisting of carbohydrates and/or proteins to increase their survival. Some studies also suggest that increased intracellular concentrations of protective solutes such as for example trehalose can also result in better survival after processing. Trehalose is a disaccharide that is accumulated intracellularly by several organisms, e.g. bacteria, yeasts and plants, under desiccation stress. Intracellular trehalose concentrations can also be increased by a mechanical method, i.e. pulsed electric field treatment. Pulsed electric field (PEF) treatment is the application of short high voltage pulses to a product between two electrodes which can result in permeabilization of the cellular membrane. PEF pre-treatment leading to increased intracellular trehalose concentrations has been shown to enhance robustness of mammalian cells towards processing, such as cryopreservation. The aim of this thesis was to develop a PEF pretreatment leading to increased intracellular protective solutes concentrations in bacteria in order to enhance survival during drying and subsequent powder storage. This aim was divided in two parts. First, to develop a PEF pre-treatment leading to increased intracellular trehalose concentrations, while maintaining culture viability. And secondly, to evaluate whether this PEF pre-treatment results in enhanced robustness during drying and subsequent powder storage.

In chapter 2 we obtained the proof of principle that PEF can be used to increase intracellular trehalose concentrations in our model bacterium Lactobacillus plantarum WCFS1. The effect of various electric field strengths was evaluated on survival and intracellular trehalose contents. Increased intracellular trehalose concentrations in L. plantarum WCFS1 were found when applying two pulses of $100 \mu$ s at electric field strengths of $7.5,10$ or $12.5 \mathrm{kV} / \mathrm{cm}$. In particular, at $7.5 \mathrm{kV} / \mathrm{cm}$ both an increased intracellular trehalose concentrations and a high survival was found. Subsequently, the fluorescent stain propidium iodide (PI) was used to study the effect of PEF treatment on the permeabilization of the cells. The stain was 
added before and after PEF treatment to evaluate whether the cellular membrane was permeabilized and whether the permeabilization was reversible or irreversible. It appeared that at $7.5 \mathrm{kV} / \mathrm{cm}$ approximately $23 \%$ of the cellular membrane was permeabilized, of which approximately half was reversibly permeabilized for PI. In chapter 3, this investigation was extended. Here, a second staining method with two impermeable stains was added; namely addition of PI before PEF treatment and addition of SYTOX Green after PEF treatment. The first method resulted in reversible permeabilized fractions up to $14 \%$ of the cell population, while the second method indicated these would be up to $\sim 40 \%$ of the population with the same PEF conditions. This difference shows that the choice of fluorescent staining technique influenced the conclusions drawn.

The second aim of this thesis was to evaluate whether a PEF pre-treatment, leading to increased intracellular trehalose concentrations, can increase robustness of bacteria during drying and subsequent powder storage. Therefore, L. plantarum cultures were PEF treated in trehalose electroporation medium and subsequently freeze dried in the same solution in chapter 4. Contrary to our hypothesis, the survival after freeze drying and subsequent powder storage did not differ between the PEF pre-treated and control cultures. Upon further studying the intracellular trehalose concentrations during the different steps of the freeze drying process we found that intracellular trehalose concentrations also increased after freezing the cells. Therefore, there was no difference in intracellular trehalose concentrations between the PEF pre-treated and control cultures anymore, which could explain the lack of difference in survival after drying and storage. Similar experiments were also performed with lactose electroporation medium. In these experiments increased intracellular lactose concentrations in L. plantarum WCFS1 were not observed after freezing, but only after freeze drying and reconstitution of the powder. This difference between intracellular trehalose and lactose accumulation indicates that potentially also biological processes play a role in the observed effects. Finally, also after spray drying increased intracellular trehalose and lactose concentrations were found upon drying in these carbohydrates.

While in chapter 4 L. plantarum WCFS1 cultures were PEF treated and subsequently dried in the same medium, in chapter 5 the cultures were PEF treated in trehalose electroporation medium and subsequently spray dried in various carrier matrices, namely trehalose, reconstituted skim milk (RSM), maltodextrin DE19 and whey protein. Interestingly, survival 
of L. plantarum WCFS1 greatly increased upon applying PEF treatment when drying in RSM. A similar increase was not observed for the other carrier matrices. Also during powder storage higher survival of PEF pre-treated cultures was found compared to control cultures without PEF when RSM was used as a carrier matrix. Furthermore, not only PEF pretreatment in trehalose electroporation medium resulted in this increases survival during spray drying in RSM, also PEF treatment in lactose electroporation medium. Analysis of intracellular trehalose and lactose concentrations before and after PEF treatment revealed that the increased robustness could not only be related to these intracellular concentrations.

The type of carrier matrix during drying influences not only the added value of a PEF pretreatment, it also affects bacterial survival during spray drying. In chapter 6 the effect of different carrier matrices on survival of L. plantarum WCFS1 during drying has been investigated by a single droplet drying approach. More specifically, particle morphology development during single droplet drying was linked to bacterial survival. Three types of particle morphologies were observed, namely dense and smooth particles, smooth and hollow particles and dented particles. Survival of L. plantarum WCFS1 was highest in carrier matrices resulting in dense and smooth particles, followed by hollow particles and lowest in dented particles. This observed relation could be explained by a combination of glass transition and diffusion dynamics in the drying droplets.

In chapter 7 the implementation of the developed PEF pre-treatment in an industrial drying process is discussed. PEF pre-treatment to enhance bacterial survival can lead to an energy reduction for drying of bacterial cultures and/or it may make a transition from the more energy and time consuming freeze drying process to spray drying possible. In addition, not only the pulse parameters described in chapter $\mathbf{2}$ and $\mathbf{3}$ can influence the outcome of a PEF treatment, also large effects of temperature and the addition of a membrane fluidizing component such as ethanol were observed.

Overall, the approach described in this thesis provides a first step towards using pulsed electric field pre-treatment, leading to increased intracellular protective solutes concentrations, to enhance bacterial robustness during drying and subsequent powder storage. Knowledge is gained on the role of intracellular protective solutes during drying of bacteria, as well as on the effect of various carrier matrices. These insights can be used to develop and optimize drying processes aimed at high bacterial culture viability. 


\section{Samenvatting}

Bacteriën worden veel gebruikt in de voedingsindustrie als startcultuur voor fermentatieprocessen of als probiotica supplementen. Voor het produceren van deze bacterieculturen worden deze eerst opgekweekt en vervolgens bewaard tot ze gebruikt worden als startcultuur of probiotica. Om de houdbaarheid van deze culturen te verlengen, worden droogprocessen zoals vriesdrogen of sproeidrogen gebruikt om een poeder te maken van deze ingrediënten. Een nadeel van het drogen van bacterieculturen is dat een aanzienlijk deel van de bacteriën deze processen niet overleeft. Daarom wordt er veel onderzoek gedaan naar het verbeteren van de overleving tijdens het drogen van culturen en de opslag van het poeder erna. Om de overleving te verbeteren worden de bacteriën vaak gedroogd in een formulering van koolhydraten en/of eiwitten. Niet alleen deze componenten aan de buitenkant van de cel kunnen een positief effect hebben op de overleving, verschillende studies laten ook zien dat verhoogde intracellulaire concentraties van zogenaamde beschermende componenten, waaronder trehalose, een positief effect kunnen hebben op overleving van bacteriën tijdens droogprocessen. Trehalose is een disacharide die ook in de natuur intracellulair gevonden wordt in verschillende organismen, waaronder bacteriën, gisten en planten, onder droogtestress.

Het verhogen van intracellulaire trehalose concentraties kan ook door een mechanische methode, pulserende elektrische velden (afgekort PEF, wat staat voor de Engelse term pulsed electric field). Een PEF behandeling bestaat uit het geven van korte (vaak microseconde) pulsen van een hoog voltage aan een product tussen twee elektroden. Deze PEF behandeling kan het celmembraan permeabel maken waardoor moleculen vanuit het medium de cel in kunnen diffunderen. Zo'n PEF voorbehandeling heeft in cellen van zoogdieren geleidt tot een verhoogde overleving na processen zoals invriezen.

Het doel van deze thesis was om een PEF voorbehandeling voor bacteriën te ontwikkelen die resulteert in verhoogde intracellulaire concentraties van beschermende componenten om zo de overleving van de bacteriën tijdens droogprocessen te verhogen. Het eerste deel van deze thesis gaat over de PEF voorbehandeling en in het tweede deel beschrijven we het effect van deze PEF voorbehandeling op de overleving tijdens het drogen.

In hoofdstuk 2 hebben we het bewijs geleverd dat PEF gebruikt kan worden voor het verhogen van intracellulaire trehalose concentraties in bacteriecellen. Dat hebben we 
gedaan in onze modelstam Lactobacillus plantarum WCFS1. Verschillende elektrische veldsterktes $(7.5,10$ en $12.5 \mathrm{kV} / \mathrm{cm})$ resulteerden in verhoogde intracellulaire trehalose concentraties bij het geven van 2 pulsen van $100 \mu \mathrm{s}$. Bij $7.5 \mathrm{kV} / \mathrm{cm}$ vonden we zowel een hoge overleving als een verhoogde intracellulaire trehalose concentratie. Het fluorescente molecuul propidiumjodide (PI) is vervolgens gebruikt om het effect van de PEF behandeling op het celmembraan verder te bestuderen. Door het toevoegen van PI voor en na PEF konden we bestuderen welk deel van de populatie een permeabel celmembraan had na PEF, en of deze permeabiliteit reversibel was of irreversibel. Na een PEF behandeling van $7.5 \mathrm{kV} / \mathrm{cm}$ had maar 23\% van de celpopulatie een permeabel membraan, waarvan ongeveer de helft reversibel permeabel was. In hoofdstuk 3 was dit onderzoek verder uitgebreid. Hier hebben we een tweede methode toegevoegd, waarbij PI voor de PEF behandeling wordt toegevoegd en een tweede membraan impermeabel molecuul, SYTOX Green, na de PEF behandeling. Waar de eerste methode resulteerde in reversibel permeabele celfracties van maximaal 14\%, resulteerde de tweede methode in grotere reversibel permeabele celfracties tot $40 \%$ van de celpopulatie wanneer dezelfde PEF condities gebruikt waren. Dit verschil laat zien dat de keuze van de methode om reversibiliteit van het celmembraan te bestuderen invloed kan hebben op de gevonden resultaten.

Het tweede doel van deze thesis was om na te gaan of de PEF voorbehandeling, die resulteerde in verhoogde intracellulaire trehalose concentraties, ook de overleving van bacteriën tijdens het drogen en/of tijdens de poeder opslag erna kan verhogen. Culturen van L. plantarum zijn daarom voorbehandeld met PEF in een trehalose oplossing en vervolgens gevriesdroogd in dezelfde oplossing in hoofdstuk 4. Tegengesteld aan onze hypothese resulteerde de PEF voorbehandeling niet in een verhoogde overleving na vriesdrogen en opslag van het poeder erna ten opzichte van controle culturen zonder PEF voorbehandeling. Door verder te kijken naar de intracellulaire trehalose concentraties tijdens de verschillende stappen van het vriesdroogproces hebben we gevonden dat de intracellulaire trehalose concentraties ook toegenomen waren na het invriezen van de cellen voor het drogen. Hierdoor was er geen verschil meer in intracellulaire trehalose concentraties tussen de controle en de PEF voorbehandelde culturen. Dit kan verklaren waarom er geen verschil in overleving gevonden is na het drogen en de poederopslag erna. Vergelijkbare experimenten zijn ook gedaan in een lactose oplossing, in plaats van trehalose. In deze experimenten vonden we geen toename in intracellulair lactose na het invriezen, maar alleen na het drogen en rehydrateren van het poeder. Dit verschil tussen 
trehalose en lactose kan betekenen dat ook biologische processen een rol spelen in de geobserveerde effecten. Niet alleen na het vriesdrogen, maar ook na sproeidrogen en rehydrateren van het poeder vonden we een toename van intracellulair trehalose en lactose als de culturen waren gedroogd in een oplossing met deze suikers.

In hoofdstuk $\mathbf{4}$ is dezelfde trehalose oplossing gebruikt voor zowel de PEF voorbehandeling als het drogen. In hoofdstuk 5 vond de PEF voorbehandeling ook plaats in de trehalose oplossing, maar zijn de culturen vervolgens gesproeidroogd in een andere formulering, namelijk opgelost melkpoeder (RSM; reconstituted skim milk), maltodextrine DE19, weieiwit en trehalose ter vergelijking. De overleving van L. plantarum na het sproeidrogen nam toe door de PEF voorbehandeling als de cultuur vervolgens gedroogd werd in RSM, terwijl dit niet het geval was in de andere formuleringen. En niet alleen na het sproeidrogen was er een voordelig effect van de PEF voorbehandeling, ook tijdens de opslag van het poeder erna bleef er een verhoogde overleving voor de PEF voorbehandelde culturen. Naast de PEF voorbehandeling in trehalose, resulteerde ook de PEF voorbehandeling in lactose in een verbeterde overleving na sproeidrogen in RSM. Na analyse van de intracellulaire trehalose en lactose concentraties voor en na de PEF behandeling kunnen we concluderen dat de verhoogde overleving niet alleen het resultaat is van de intracellulaire concentraties van deze componenten.

De formulering tijdens drogen heeft niet alleen effect op of de PEF voorbehandeling wel of geen toegevoegde waarde heeft, maar de formulering zelf heeft ook invloed op de overleving tijdens het sproeidrogen. In hoofdstuk 6 hebben we het effect van verschillende formuleringen bestudeerd met behulp van een druppeldroger. Hiermee is de morfologie van een gedroogd deeltje gelinkt aan de overleving van L. plantarum WCFS1 in deze formulering. De drie soorten morfologieën na het drogen zijn kleine dichte deeltjes met een glad oppervlak, holle deeltjes met een glad oppervlak en ingedeukte deeltjes. De overleving van L. plantarum was het hoogst in de kleine dichte deeltjes, gevolgd door de holle deeltjes en het laagst in de ingedeukte deeltjes. Dit kon verklaard worden door een combinatie van glasvorming en diffusiedynamiek in de druppels tijdens het droogproces.

In hoofdstuk 7 staat de implementatie van de PEF voorbehandeling in het industrieel droogproces van bacterieculturen beschreven. De PEF voorbehandeling om bacteriën deze processen beter te laten overleven kan leiden tot een reductie in de energie die nodig is voor de droogprocessen en/of een overgang van het energie- en tijdrovende 
vriesdroogproces naar sproeidrogen mogelijk maken voor bacterieculturen. Verder waren er naast de PEF condities die in hoofdstuk $\mathbf{2}$ en $\mathbf{3}$ beschreven zijn ook grote effecten van de temperatuur en het toevoegen van membraan fluïdiserende componenten zoals ethanol op de intracellulaire trehalose concentraties na PEF behandeling.

Uiteindelijk is de methode die beschreven is in deze thesis een eerste stap naar het gebruik van een PEF voorbehandeling, die resulteert in een toename van intracellulaire beschermende componenten, voor het verhogen van de overleving van bacterieculturen tijdens drogen en poeder opslag. Verder is er kennis gegenereerd over de rol van intracellulaire beschermende componenten tijdens drogen en over het effect van verschillende formuleringen tijdens het droogproces. Deze inzichten kunnen gebruikt worden voor de ontwikkeling en optimalisatie van droogprocessen die leiden tot een hoge overleving van bacterieculturen. 


\section{Acknowledgements}

About the author

\section{Publications}

\section{Overview of completed training activities}





\section{Acknowledgements}

I find it very strange to write these acknowledgements now from home, in a time where we are all working at home. It also makes me realize even more how much I enjoyed my time as a PhD at FPE and FHM and how many nice and inspiring people I met during this time!

First, I would like to thank my supervisors. Maarten, dankjewel voor al je support, je deur die altijd open stond voor kleine en grotere vragen en je positieve motivatie op de momenten dat ik het nodig had. Heidy, van jouw oog voor details en kritische blik heb ik veel geleerd. Dankjewel voor al je support! Samen waren jullie een top supervisor team dat elkaar goed aanvulde; met Maarten die de grote lijnen in de gaten hield en Heidy met haar kritische blik als ik soms iets te snel wilde. Remko, ik wil ook jou bedanken voor al je enthousiasme en feedback in het begin en eind van mijn PhD.

Onmisbaar voor het PEF deel van mijn werk was de samenwerking met Rian en Erik. Rian, dankjewel voor al het meedenken over het PEF onderzoek, door jouw kennis hebben we hier grote stappen in kunnen maken. Erik, regelmatig raakte ik je even kwijt als het ging over de technische termen van de elektrische circuits achter het PEF apparaat, maar zonder jou was de batch PEF cuvet er niet gekomen en hadden we deze experimenten niet zo kunnen doen. I would also like to thank all other project partners of the ISPT project (Nutricia, DSM, NIZO, WFBR, Winclove and Cosun) and the ISPT office. I appreciated our meetings a lot and learned so much from all your critical questions and the discussions that we had during this project!

This PhD thesis contains a lot of experimental work, it would not have been possible to do this all alone. Luckily there were many students that worked together with me on this project: Jan, Tiffany, Isabel, Niels, Sylvia, Letao, Jiska, Hilde, Paul, Sietske, Tianze and Kevin, thank you all so much for your enthusiasm for this project and your hard work. I learnt a lot from all of you and enjoyed working together in the lab. Isabel, heel fijn dat we vanuit jouw thesis en onze PhD projecten zo veel samen hebben kunnen werken, het is dan ook niet meer dan logisch dat je mijn paranimf bent!

Then there are also several people that are essential for FPE \& FHM for answering numerous smaller and bigger questions, for help with new methods and arranging several things. Maurice, Martin, Jos, Ingrid, Judith, Wouter, Jarno, Marjan, Ilona, Gerda en Marcel T; heel 
veel dank daarvoor. Marcel, dankjewel voor al je tijd en moeite om samen de flow cytometrie experimenten uit te voeren.

Special thanks to all my office mates; Izabella, Pina, Angelica, Jan-Eise, Jun, Wanqing, Sten, Jaap and Anja. I always enjoyed our talks between the work, even if it was via Skype these last months. Anja, van het begin tot aan het eind van mijn PhD zaten we in hetzelfde kantoor en hebben we veel PhD ervaringen en leuke activiteiten samen meegemaakt, heel fijn dat je dan ook nu mijn paranimf bent.

My PhD time would not have been so much fun without all the activities together with colleagues; coffee breaks, lunch walks, funXtion trainings, borrels, the weekly running trainings, Veluweloop, lab outings, WE-day, PhD trips and so much more. I would like to thank the lunch walk and sports group for having our active breaks; Maurice, Birgit, Marlies, Eline, Isabel, Melanie, Anja, Regina, Luc, Joanne and many others. Emma, Floor and Qinhui; thanks for allowing me to come to your office for some chitchats. Eline, Nynke en Anja, dankjewel voor onze mooie reis in Canada! Thanks to my fellow PhD trip committee members Natalia, Jeroen and Oscar, I enjoyed our trip to Italy and organizing it a lot. I would also like to thank you for your help and nice talks in the FHM labs, also Yue, Maren, Jasper, Pjotr and Rebecca. Thanks to the running group for all the fun and for keeping my $5 \mathrm{k}$ condition up to date even when the weather was bad; Frank, Marcel Z, Dennis, Esther, Marcel T, Nathalia, Denja, Oscar, Jeroen, Bernhard and James Noah. Thanks to all other colleagues who made the PhD trips, borrels, breaks and other activities so much fun: Steven, Jan, Lu, Sicong, Juliana, Fiona, Eric, Patricia, Katharina, Konstantina, Andrea, Murat, Bijoy, Ivanna, Zulhaj, Anna, Yu, Anouk, Alberto and many more.

Natuurlijk kan ik mijn lieve vrienden en familie niet vergeten. Mijn Limburgse chickies, Rosita en Kleinmaerfijn; dankjewel dat jullie de leuke en minder leuke verhalen van mijn $\mathrm{PhD}$ wilden aanhoren en er altijd waren om bij te kletsen en leuke dingen te doen! Roelie, Hans, Ellen en Jeroen, dankjewel voor al jullie support. Pap, mam, Moniek, Sidney, Loes en Marcel, ook al snapten jullie niet altijd wat ik nu precies deed met die gekke bacteriën, ik weet dat jullie me altijd steunen en trots op me zijn, dat waardeer ik enorm!

Zoals ik dit dankwoord begonnen ben met een Maarten bedanken, eindig ik het ook met een Maarten bedanken, gelukkig weten ze zelf wel wie ik bedoel ;). Lieve Maarten, dankjewel dat je er altijd voor me bent. 


\section{About the author}

Evelien Vaessen was born on the $31^{\text {st }}$ of July 1992 in Heerlen, The Netherlands. She attended Sintermeerten College in Heerlen, where she obtained her VWO diploma in 2010, with majors in Natuur \& Techniek (Nature \& Engineering) and Natuur \& Gezondheid (Nature \& Health). After this she started her study Biotechnology at Wageningen University. She finished her bachelor studies with a thesis at the laboratory of Bioprocess

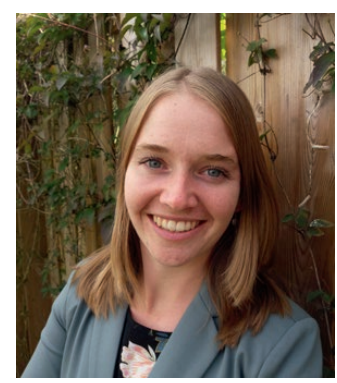
Engineering in 2013 about the effect of lignocellulose derived compounds on Bacillus growth and acid production.

In the same year Evelien continued with her master studies in Biotechnology, with a specialization in Process Technology. For her master thesis she worked on modelling and bioreactor experiments to understand the cell cycle of microalgae at the laboratory of Bioprocess Engineering. After finishing this thesis, Evelien moved to Portugal for her internship at research institute iBET in Oeiras, where she worked on upstream process optimization of insect cell cultures for the production of Influenza virus-like particles. She graduated for her master in 2015.

She started as a PhD candidate at the laboratories of Food Process Engineering and Food Microbiology in 2016. Her research focused on a pulsed electric field pre-treatment to increase survival of probiotics and starter cultures during drying, and the results of this work are described in this thesis.

Contact: emj.vaessen@gmail.com 



\section{Publications}

van der Pol, E. C., Vaessen, E., Weusthuis, R. A., \& Eggink, G. (2016). Identifying inhibitory effects of lignocellulosic by-products on growth of lactic acid producing microorganisms using a rapid small-scale screening method. Bioresource Technology, 209, 297-304.

de Winter, L., Cabanelas, I. T. D., Órfão, A. N., Vaessen, E., Martens, D. E., Wijffels, R. H., \& Barbosa, M. J. (2017). The influence of day length on circadian rhythms of Neochloris oleoabundans. Algal Research, 22, 31-38.

Vaessen, E. M. J., den Besten, H. M. W., Patra, T., van Mossevelde, N. T. M., Boom, R. M., \& Schutyser, M. A. I. (2018). Pulsed electric field for increasing intracellular trehalose content in Lactobacillus plantarum WCFS1. Innovative Food Science \& Emerging Technologies, 47, 256-261.

Schutyser, M. A. I., Both, E. M., Siemons, I., Vaessen, E. M. J., \& Zhang, L. (2019). Gaining insight on spray drying behavior of foods via single droplet drying analyses. Drying Technology, 37(5), 525-534.

Vaessen, E. M. J., den Besten, H. M. W., Esveld, E. D. C., \& Schutyser, M. A. I. (2019). Accumulation of intracellular trehalose and lactose in Lactobacillus plantarum WCFS1 during pulsed electric field treatment and subsequent freeze and spray drying. LWT, 115, 108478.

Vaessen, E. M. J., Timmermans, R. A. H., Tempelaars, M. H., Schutyser, M. A. I., \& den Besten, H. M. W. (2019). Reversbility of membrane permeabilization in Lactobacillus plantarum WCFS1 after pulsed electric field treatment. Scientific Reports, 19990.

Siemons, I., Vaessen, E. M. J., Oosterbaan van Peski, S. E., Boom, R. M., \& Schutyser, M. A. I. (2021) Protective effect of carrier matrices on survival of Lactobacillus plantarum WCFS1 during single droplet drying explained by particle morphology development. Journal of Food Engineering, 292, 110263.

Vaessen, E. M. J., den Besten, H. M. W., Leito, K. M. N., \& Schutyser, M. A. I. Pulsed electric field pre-treatment for enhanced bacterial robustness during drying: Effect of carrier matrix and strain variability. Submitted for publication.

Vaessen, E. M. J., Kemme, H.A., Timmermans, R. A. H., Schutyser, M. A. I., \& den Besten, H. M. W. Temperature and presence of ethanol affect accumulation of intracellular trehalose in Lactobacillus plantarum WCFS1 upon pulsed electric field treatment. Submitted for publication. 



\section{Overview of completed training activities}

\section{Discipline specific activities}

Courses

$3^{\text {rd }}$ School for Pulsed Electric Field processing of Foods (UCD Dublin, IR) 2016

Reaction Kinetics in Food Science (VLAG, Wageningen, NL) 2016

Genetics and physiology of food-associated microorganisms (VLAG, 2016 Wageningen, $\mathrm{NL}$ )

$\begin{array}{ll}\text { Spray drying (NIZO, Ede, NL) } & 2017\end{array}$

The intestinal microbiome and Diet in Human and Animal Health (VLAG, 2017 Wageningen, $\mathrm{NL})^{\text {a }}$

Numerical methods for chemical engineers (OSPT, Eindhoven, NL) 2017

Radiation Protection 5B (Boerenhaave Nascholing, Leiden, NL) 2017

Conferences

NWGD symposium (Wageningen, $\mathrm{NL})^{\mathrm{a}}$

2016-2018

Eurodrying (Liege, BE) ${ }^{a}$

2017

Food Structure and Functionality Forum (Montreal, CA) a,b 2018

$\begin{array}{ll}\text { Eurodrying (Turin, IT) } & \\ & \text { b }\end{array}$

World Congress on Electroporation (Toulouse, FR) ${ }^{\text {b }} 2019$

$\begin{array}{ll}\text { Effost conference (Rotterdam, NL) } & \\ \end{array}$

\section{General courses}

$\begin{array}{ll}\text { VLAG PhD week (VLAG) } & 2016\end{array}$

Competence Assessment (WGS) 2016

Wageningen PhD carousel (WGS) 2016

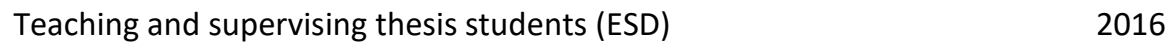

$\begin{array}{ll}\text { Scientific publishing (WGS) } & 2017\end{array}$

$\begin{array}{ll}\text { Scientific writing (WGS) } & 2018\end{array}$

Scientific artwork (WUR library) 2018

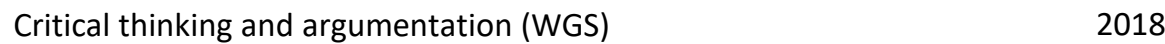

$\begin{array}{ll}\text { Career perspectives (WGS) } & 2019\end{array}$ 
Optional courses and activities

FPE \& FHM weekly meetings ${ }^{b}$

2016-2020

FPE groupdays ${ }^{a, b}$

2016-2019

VLAG PhD council

2016-2019

FHM PhD study tour Italy ${ }^{b}$

2017

Organisation FHM PhD study tour Italy

2017

FPE PhD study tour Canada ${ }^{a}$

2018

${ }^{a}$ Poster presentation, ${ }^{b}$ Oral presentation

NL: The Netherlands, IR: Ireland, BE: Belgium, CA: Canada, IT: Italy, FR: France 

The work presented in this thesis has been carried out in the framework of the Institute of Sustainable Process Technology (ISPT) under the project "DR-20-06: Low energy living bacterial food formulations". This project is co-funded by TKI-E\&I with the supplementary grant 'TKI- Toeslag' for Topconsortia for Knowledge and Innovation (TKI's) of the Ministry of Economic Affairs and Climate Policy.

Cover design by Evelien Vaessen and Dennis Hendriks

Printed by Digiforce || ProefschriftMaken 

